\title{
Cardiac adaptation during and after hypertensive gestation
}

Citation for published version (APA):

Ghossein-Doha, C. (2015). Cardiac adaptation during and after hypertensive gestation. [Doctoral Thesis, Maastricht University]. Datawyse / Universitaire Pers Maastricht. https://doi.org/10.26481/dis.20150417cg

Document status and date:

Published: 01/01/2015

DOI:

$10.26481 /$ dis.20150417cg

Document Version:

Publisher's PDF, also known as Version of record

\section{Please check the document version of this publication:}

- A submitted manuscript is the version of the article upon submission and before peer-review. There can be important differences between the submitted version and the official published version of record.

People interested in the research are advised to contact the author for the final version of the publication, or visit the DOI to the publisher's website.

- The final author version and the galley proof are versions of the publication after peer review.

- The final published version features the final layout of the paper including the volume, issue and page numbers.

Link to publication

\footnotetext{
General rights rights.

- You may freely distribute the URL identifying the publication in the public portal. please follow below link for the End User Agreement:

www.umlib.nl/taverne-license

Take down policy

If you believe that this document breaches copyright please contact us at:

repository@maastrichtuniversity.nl

providing details and we will investigate your claim.
}

Copyright and moral rights for the publications made accessible in the public portal are retained by the authors and/or other copyright owners and it is a condition of accessing publications that users recognise and abide by the legal requirements associated with these

- Users may download and print one copy of any publication from the public portal for the purpose of private study or research.

- You may not further distribute the material or use it for any profit-making activity or commercial gain

If the publication is distributed under the terms of Article $25 \mathrm{fa}$ of the Dutch Copyright Act, indicated by the "Taverne" license above, 


\section{Cardiac Adaptation During and After Hypertensive Gestation}

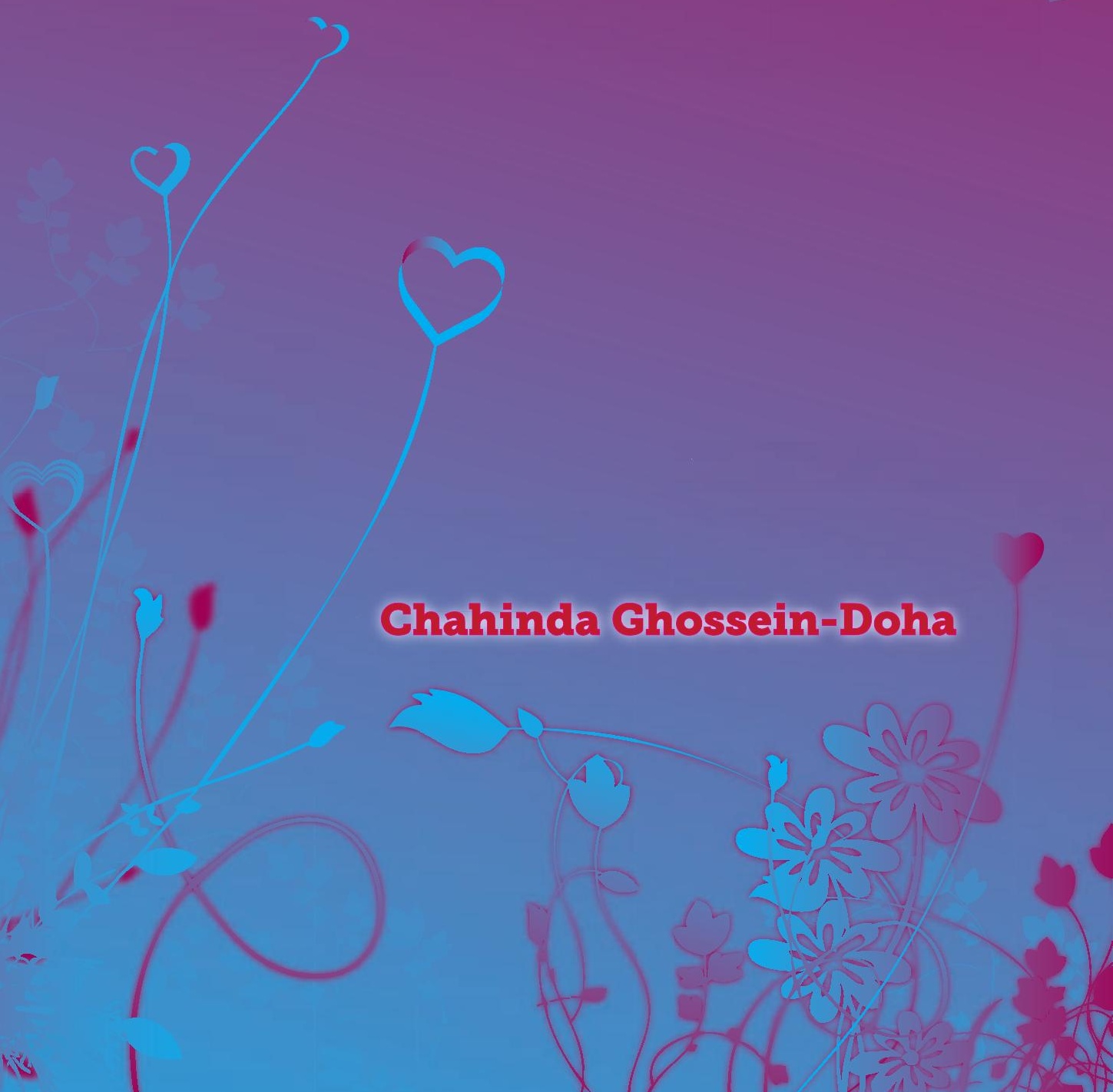


ISBN 9789461594280

Cover design: Stefan Fincken www.stefanfincken.com Layout: Yvonne Leenders

Printed by: Datawyse / Universitaire Pers Maastricht 


\title{
Cardiac Adaptation During and After Hypertensive Gestation
}

\author{
PROEFSCHRIFT \\ ter verkrijging van de graad van doctor aan de Universiteit Maastricht, \\ op gezag van de Rector Magnificus, Prof. Dr. L.L.G. Soete \\ volgens het besluit van het College van Decanen, \\ in het openbaar te verdedigen \\ op vrijdag 17 april om 10:00 uur \\ door \\ Chahinda Ghossein-Doha
}

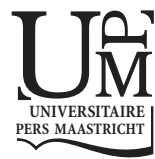




\section{Promotor}

Prof. dr. M.E.A. Spaanderman

\section{Copromotor}

dr. L.L.H. Peeters

\section{Beoordelingscommissie}

Prof. dr. P.W. de Leeuw (voorzitter)

Prof. dr. H.P Brunner-La Rocca

Dr. S.V. Koenen (Utrecht University Medical Center)

Prof. dr. A. H. E. M. Maas (Radboud University Medical Center)

Prof. dr. J.G. Nijhuis

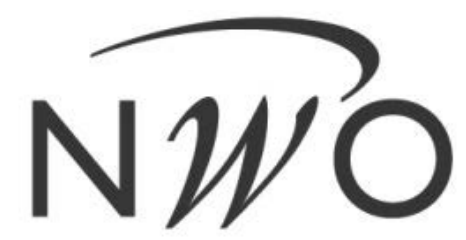

Netherlands Organisation for Scientific Research

"This research was financially supported by the Netherlands Organisation for Scientific Research (NWO) in the framework of the Mosaic, project 017.009.127"

"Financial support by the Dutch Heart Foundation for the publication of this thesis is gratefully acknowledged"

"Financial support by the Stichting HELLP-syndroom for the publication of this thesis is gratefully acknowledged" 
Dit proefschrift draag ik op aan ons lief engeltje Rachelle. 



\section{Table of content}

Chapter 1 General Introduction

Part 1 Cardiac adaption after preeclampsia

Chapter 2 Cardiac adaptation to the next pregnancy in formerly preeclamptic women bound to develop recurrent preeclampsia

Chapter 3 Age-related alterations in cardiac geometry in formerly preeclamptic women and healthy parous controls; an explorative study

Part 2 Risk factors for hypertension and heart failure stage-B after preeclampsia

Chapter 4 Hypertension after preeclampsia is preceded by changes in cardiac structure and function

Chapter 5 Long-term risk to develop hypertension in formerly

preeclamptic women: A longitudinal pilot study

Chapter 6 Preeclampsia, an independent risk factor for asymptomatic heart failure in young women

Chapter 7 General Discussion and Summary

Chapter 8 Valorisatie

Nederlandstalige samenvatting

Dankwoord 


\section{Abbreviations \& Definitions}

\section{Abbreviations}

CVD

HF stage-B

HT

LVH

LVM

LVR

PE

\section{Definitions}

Early-onset PE

HELLP

Hypertension after pregnancy

Late-onset PE

Preeclampsia (PE)

Preexistent hypertension

Pregnancy induced hypertension

Prehypertension

Recurrent PE

Superimposed PE
Cardiovascular Diseases

Heart Failure Stage B

Hypertension

Left Ventricular Hypertrophy

Left Ventricular Mass

Left Ventricular Remodeling

Preeclampsia

Preeclampsia diagnosed after 20 weeks but before 34 weeks of gestation

The hypertensive pregnancy disorder characterized by: Hemolysis, Elevated Liver enzymes and Low Platelet count

Hypertension ( $\geq 140 / 90 \mathrm{mmHg}$ ) and/or usage of antihypertensive drugs after first completed pregnancy

Preeclampsia diagnosed after 34 weeks of gestation

A syndrome of pregnancy characterized by newonset hypertension ( $\geq 140 / 90 \mathrm{mmHg}$ ) and proteinuria ( $\geq 300 \mathrm{mg} / 24 \mathrm{hr}$ ); diagnosed after 20 weeks of gestation

Hypertension ( $\geq 140 / 90 \mathrm{mmHg}$ ) and/or usage of antihypertensive drugs before pregnancy; or hypertension ( $\geq 140 / 90 \mathrm{mmHg}$ ) diagnosed before 20 weeks of gestation

New-onset hypertension diagnosed after 20 weeks of gestation without concomitant proteinuria

Systolic blood pressure 120-139 mmHg and/or diastolic blood pressure of $80-89 \mathrm{mmHg}$.

Preeclampsia complicating a first and a second pregnancy

PE developing in women with preexistent hypertension 


\section{Chapter 1}

\section{General introduction}

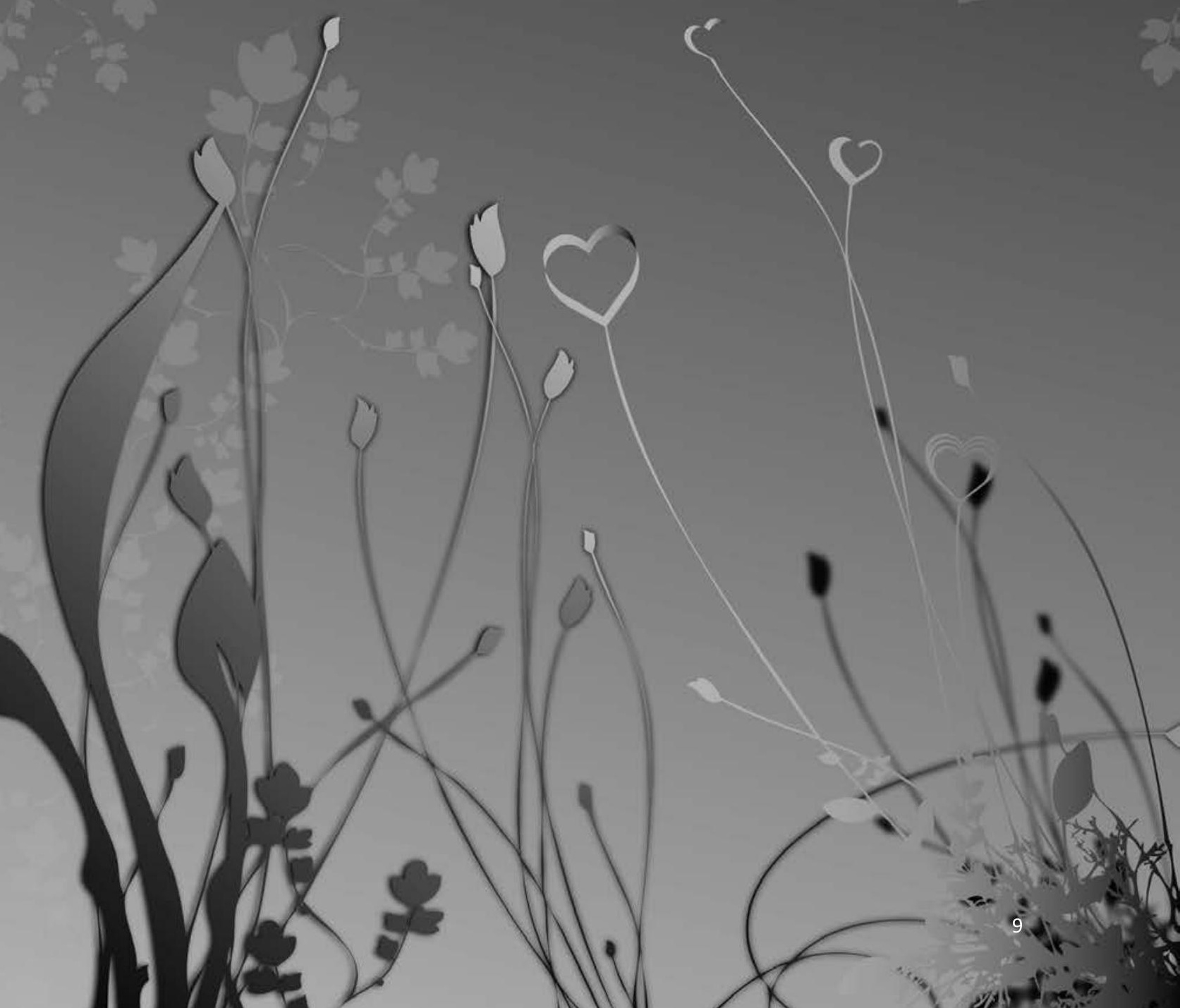


Preeclampsia (PE), a hypertensive pregnancy disorder complicating $3-5 \%$ of all pregnancies in developed countries, is a major cause of perinatal morbidity and mortality.1 Although a link between PE and future chronic hypertension (HT) was already suggested almost 50 years ago, 2 the disorder has only recently been accepted to predispose to chronic HT and premature cardiovascular disease (CVD).3 PE and CVD are linked to one another by sharing many risk conditions such as diabetes, obesity, and preexistent $\mathrm{HT}$, which suggests overlap in their pathogenesis.

Chronic HT itself is a major predictor of cardiovascular disease in the general population, contributing about $30 \%$ to the risk of myocardial infarction in women. 4 Chronic HT may be an important mediator in the development of cardiovascular disease in women with a history of PE.

During $\mathrm{PE}$ the maternal heart remodels by concentric left-ventricular hypertrophy (LVH).5,6 PE-induced concentric LVH does not always resolve completely. In fact, up to 1 year postpartum, and more often after early- than after late-onset PE, many former patients present with increased left ventricular mass index (LVMi) along with (asymptomatic) stage-B heart failure (HF).7 It is still obscure whether this protracted increase in LVMi: 1) eventually resolves completely in the subsequent years, 2) expedites normal cardiac aging, 3) raises the risk of recurrent PE in a next pregnancy, and/or 4) increases the risk of developing chronic HT and HF later in life.

\section{Women and cardiovascular disease}

In Western countries, more women die of CVD than of any other disease ${ }^{8-10}$ making CVD in women an important public health issue. Conventional risk factors, such as insulin resistance, obesity, dyslipidaemia and a positive family history for CVD, do not differ between men and women although gender differences may prevail in the magnitude of some effects. ${ }^{11}$ Meanwhile, female-specific risk conditions, such as PE ${ }^{12}$ and polycystic ovary syndrome, ${ }^{13}$ are also associated with a higher risk of premature CVD with PE increasing the risk of CVD two- to fourfold later in life. ${ }^{12,14-16}$

$\mathrm{HF}$ is categorized by The American College of Cardiology/American Heart Association (ACC/AHA) in 4 stages (Fig. 1). ${ }^{17}$ The preclinical stages $A$ (presence of risk factors) and $B$ (functional alterations) are considered to form a continuum preceding the development of the clinical HF stages $C$ and $D$ (symptomatic heart disease). ${ }^{17}$ The progression from the preclinical stage $B$ to the clinical stage $C$ is associated with a 5fold rise in cardiovascular-related mortality, ${ }^{18,19}$ decreased quality-adjusted life years. ${ }^{19}$ Early detection and tailored intervention in women with stage-B HF postpones progression to stage $\mathrm{C}$, thus improving clinical outcome and lowering cardiovascularrelated morbidity and mortality. ${ }^{18,19}$

Whether the reported high prevalence of stage-B HF in formerly preeclamptic women at one year postpartum reflects an end stage, or rather an intermediate stage in a slowly resolving process is still unsettled. Even though, this young female risk population deserves cardiovascular risk management to prevent early-onset HF. 


\section{Basic aspects of left-ventricular hypertrophy}

Cardiac remodeling (LVR) is defined as the change in cardiac size, shape and/or function in response to chronic cardiac volume or pressure overload. ${ }^{20-22}$ Left ventricular hypertrophy (LVH) implies that size and total mass of the myocytes of the left ventricle are increased. It forms a fundamental adaptive process to an increased mechanical stress accompanying increased workload. ${ }^{23}$ Development of cardiac hypertrophy is essentially beneficial since it augments the number of contractile units and reduces ventricular wall stress to normal levels according to the law of Laplace. ${ }^{23}$ Even though the adaptive changes do have some functional benefits, it goes at the expense of an increased risk of heart failure, arrhythmias and ischaemic heart disease.

Chronic volume and pressure overload promote protein synthesis, enlarge the sarcomeres within individual myocytes and alter their organization ${ }^{24}$ They also trigger distinct patterns of gene expression resulting in various types of remodeling. ${ }^{25,26}$ Recent evidence suggests that these two types of stimuli also determine the resulting phenotype of the hypertrophic remodeling. ${ }^{25}$

Cardiac remodeling may be physiologic (eccentric hypertrophy) or pathologic (concentric hypertrophy). ${ }^{20,22}$ Eccentric LVH indicates a concerted increase in leftventricular end-diastolic diameter and wall thickness and represents a healthy cardiovascular adaptive response to the strain of training as observed in athletes and healthy pregnancy. This type of remodeling enables the heart to function more efficiently during normal pregnancy at given systolic and diastolic functions. ${ }^{20}$ Ventricular dilation occurs as new sarcomeres are added in-series to existing sarcomeres. ${ }^{27}$ This form of LVR serves to diffuse wall tension over more muscle fibres (sarcomere units) thus diminishing the (primarily volume-load related) stress exerted upon the ventricular wall. ${ }^{20,28}$ In contrast, concentric LVR occurs when the left ventricle adapts to a chronically raised pressure overload (i.e. cardiac afterload) as occurs during chronic HT, aortic valve stenosis and PE. ${ }^{7,20,28}$ This type of LVR evolves without concomitant widening of the left ventricle but with substantial increase of wall thickness as new sarcomeres are added in parallel to existing sarcomeres. ${ }^{27}$ In contrast to eccentric LVR, ${ }^{29}$ the cardiac myocyte hypertrophy in concentric LVR is typically accompanied by connective tissue accretion, mostly consisting of collagen and fibroblasts. ${ }^{27}$ The accumulation of myocardial collagen increases tissue stiffness and with it reduces mechanical efficiency. The additional increase in LVM in PE on top of that induced physiologically in the pre-disease stage of pregnancy $y^{5,30}$ may not always resolve postpartum. ${ }^{7}$ PE-related residual LVH observed in these relatively young women at one year postpartum, may predispose to stage-B HF. ${ }^{7}$

\section{Clinical presentation of preeclampsia}

To date, $\mathrm{PE}$ is still managed as a clinical syndrome with largely unknown pathogenesis. $\mathrm{PE}$ is diagnosed on the basis of new-onset $\mathrm{HT}$ accompanied by new-onset proteinuria after the $20^{\text {th }}$ week of pregnancy. ${ }^{31}$ Superimposed PE indicates that a woman with chronic HT has developed new-onset proteinuria in the second half of pregnancy. Eclampsia refers to the de-novo development of seizures during pregnancy, almost always in women previously diagnosed with PE (Table 1). Nowadays, PE is considered 
to have implications extending to beyond the transient episode of disease during pregnancy. In the last two decades, PE has been accepted as a multisystem disorder triggered by placental dysfunction, typically accompanied by excessive placental shedding of reactive oxygen species (ROS) and other endotheliotoxic products into the maternal blood perfusing the intervillous space. ${ }^{32}$ These toxic compounds induce (systemic) endothelial dysfunction giving rise to symptoms such as HT, albuminuria, enhanced systemic vasoconstriction, increased vascular permeability giving rise to generalized edema, activated hemostasis and inflammatory response, and cardiovascular sympathetic over-activity. ${ }^{33,31}$

It is conceivable that the PE-induced damage of the maternal cardiovascular system by the toxic placental products - possibly in concert with the mechanical strain associated with the transient HT - is only partly reversible and therefore, responsible for the increased risk of premature maternal CVD. ${ }^{12}$ However, the currently generallyaccepted view is that common pre-existing metabolic and cardiovascular risk factors contribute to the increased risk of PE and premature CVD, which may have partly overlapping pathogenetic features.

Because of poor insight in its cause(s), PE is still defined on the basis of clinical criteria only. In spite of the (by definition) homogeneous clinical presentation of the disorder, there is convincing evidence for PE to be the clinical endpoint of different pathogenetic pathways. ${ }^{34}$ More specifically, various triggers - alone or combined - may initiate the pathogenetic cascade that eventually lead to PE. It follows that PE is a heterogeneous system biology disorder consisting of various subgroups, which ought to be managed differently taking into account, not only gestational age and rate of clinical deterioration, but also - if possible - the initial phase of the pathogenesis. The latter has brought various experts to propose a number of more homogenous sub-groups of PE. ${ }^{34}$ Some affected women develop early-onset (<34 weeks), others late-onset PE (>34 weeks), with a wide variation in severity independent of gestational age of onset. Of all nulliparous former PE patients, only about $25 \%{ }^{1}$ is destined to develop recurrent $P E$ in their next pregnancy. It is conceivable that the pathogenesis of PE differs between former patients who do and do not develop a recurrence, presumably also reflected in differences in the early-pregnancy cardiovascular adaptation. This raises the question as to whether the cardiac adaptive response to the next pregnancy differs between former early-onset PE patients developing recurrent PE and their counterparts who have a healthy course and outcome of their next pregnancy.

With respect to demography, it is well-known that women with one or more features of the metabolic syndrome are at increased risk to develop PE. ${ }^{35,36}$ This also applies to women with pre-existing (subclinical) diabetes and the antiphospholipid syndrome. ${ }^{37}$ These disorders often remain undiagnosed in the absence of clinically relevant symptoms. Subdivision of PE by clinical criteria without taking into account the heterogeneous pathogenesis can also be expected to lead to conflicting results in epidemiologic studies on PE due to differently-composed study populations. 


\section{Preeclampsia and the heart}

In the first trimester of human pregnancy, cardiac output increases by about $40 \%$ in concert with a fall in total peripheral vascular resistance ${ }^{38}$ and a rise in cardiac preload. $^{39}$ In healthy pregnancies, these first-trimester hemodynamic changes are accompanied by eccentric cardiac remodeling. ${ }^{5,40}$ This physiologic LVR resolves in the first weeks postpartum. ${ }^{41}$ Pregnant women who develop PE elicit an additional concentric LVR, presumably secondary to the raised cardiac afterload in absence of adequate preload. ${ }^{42}$ The latter has been reported to persist for at least several months postpartum. ${ }^{7}$ Particularly early-onset PE relates to persistent postpartum residual concentric LVR. ${ }^{7}$

There is also evidence for persisting asymptomatic diastolic and systolic cardiac dysfunction, in the immediate postpartum period, a condition accompanied by elevated circulating levels of cardiovascular biomarkers, such as BNP., 6,7, 43 It is still obscure whether these abnormalities are associated with recurrent PE in a next pregnancy, or whether they contribute to the increased risk of premature development of HT and CVD later in life. ${ }^{7,12}$.

Despite the profound effects of PE on the echocardiographic indices in the clinical phase, only few studies explored cardiac geometry and function for an extended postpartum period. Recent guidelines have highlighted the importance of asymptomatic LV dysfunction and hypertrophy in the risk stratification for CVD and $\mathrm{HF}^{17}$ An increase in LVM may persist or even progress to concentric LVH in cases of elevated blood pressure. ${ }^{18,44}$ Persistent HT after PE, even when the latter subsides to prehypertension, ${ }^{45}$ can be expected to amplify the post-PE residual concentric LVH. In this context it is relevant to mention that an increase in LVM appears to be reversible with timely antihypertensive treatment. ${ }^{45}$ It follows that early identification of women with persisting postpartum structural cardiac abnormalities enables timely secondary prevention of premature CVD. 

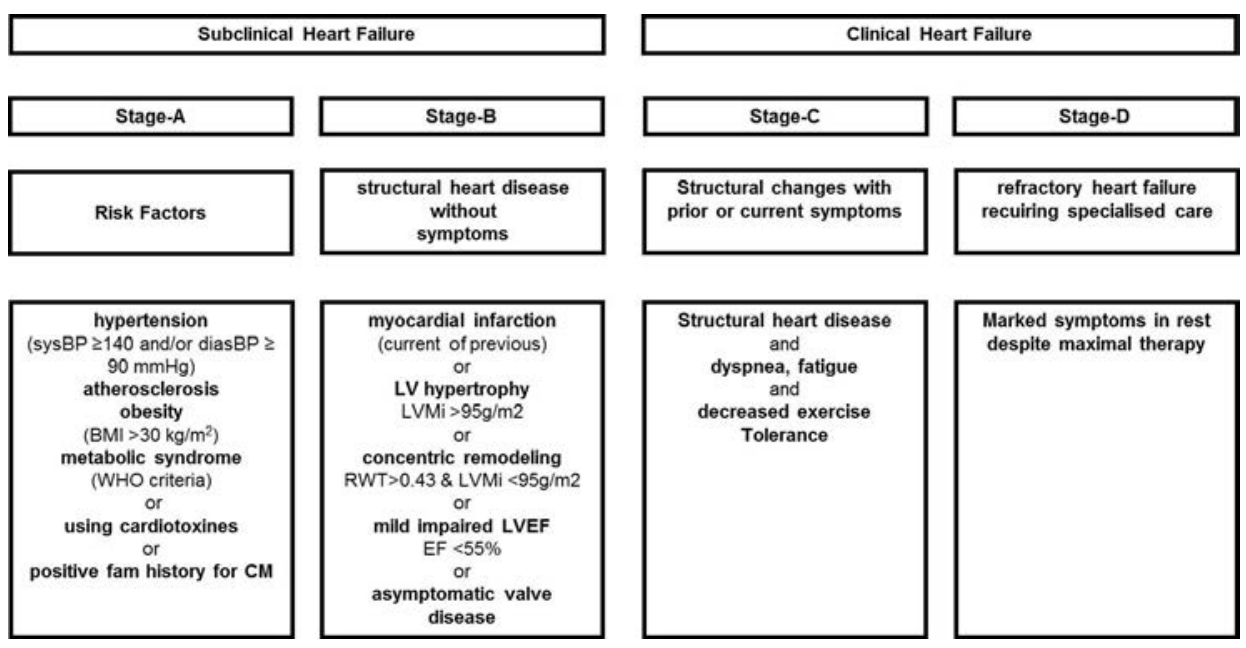

Figure 1: stages for Heart Failure, sysBP: Systolic Blood Pressure; diasBP: Diastolic Blood Pressure; WHO: World Health Organisation; CM: Cardio myopathy; LVMi: Left Ventricular Mass index; RWT: Relative Wall Thickness; LVEF: Left Ventricular Ejection Fraction.

\section{Outline of this thesis}

This thesis consists of two parts. In the first part, we explored in former PE patients the cardiac adaptive response to the next pregnancy and secondly, the age-related change in cardiac geometry. In the second part, we explored to what extent the residual postPE LVR along with well-known cardiovascular risk factors in former PE patients relate to the development of chronic HT and/or HF stage-B, later in life.

Chapter $\mathbf{2}$ describes the results in formerly early-onset PE patients, about whether recurrent $\mathrm{PE}$ in the next pregnancy is preceded by more deviant preconceptional echocardiographic indices and/or by an abnormal initial pattern of cardiac adaptation to pregnancy. Chapter 3 presents the age-related change in cardiac geometry and function in these former patients relative to parous controls between 1 and 14 years postpartum. Chapter $\mathbf{4}$ evaluates whether chronic HT develops preferentially in initially normotensive former patients with altered cardiac geometry and function at the oneyear postpartum screening. Chapter 5 presents the results of a longitudinal pilot study exploring the efficacy of cardiovascular and metabolic screening of formerly preeclamptic women at one-year postpartum to identify women destined to develop chronic HT in the subsequent 13 years. Chapter 6 explored the prevalence of stage B $\mathrm{HF}$ in formerly PE patients and healthy parous controls 5-10 years postpartum and the specific risk factors associated with the occurrence of this asymptomatic stage. Chapter 7 summarizes and elaborates on the most important results of the studies in this thesis. Finally, Chapter $\mathbf{8}$ elaborates on the contribution of the previous studies on valorisation. 


\section{References}

1. Hernandez-Diaz S, Toh S, Cnattingius S. Risk of pre-eclampsia in first and subsequent pregnancies: prospective cohort study. BMJ. 2009;338:b2255.

2. Adams EM, Macgillivray I. Long-term effect of preeclampsia on blood-pressure. Lancet. Dec 23 1961;2(7217):1373-1375.

3. Carty DM, Delles C, Dominiczak AF. Preeclampsia and future maternal health. J Hypertens. Jul;28(7):1349-1355.

4. Yusuf S, Hawken S, Ounpuu S, et al. Effect of potentially modifiable risk factors associated with myocardial infarction in 52 countries (the INTERHEART study): case-control study. Lancet. Sep 11-17 2004;364(9438):937-952.

5. Simmons LA, Gillin AG, Jeremy RW. Structural and functional changes in left ventricle during normotensive and preeclamptic pregnancy. Am J Physiol Heart Circ Physiol. Oct 2002;283(4):H1627-1633.

6. Melchiorre K, Thilaganathan B. Maternal cardiac function in preeclampsia. Curr Opin Obstet Gynecol. Dec 2011;23(6):440-447.

7. Melchiorre K, Sutherland GR, Liberati M, Thilaganathan B. Preeclampsia is associated with persistent postpartum cardiovascular impairment. Hypertension. Oct 2011;58(4):709-715.

8. Perk J, De Backer G, Gohlke H, et al. European Guidelines on cardiovascular disease prevention in clinical practice (version 2012). The Fifth Joint Task Force of the European Society of Cardiology and Other Societies on Cardiovascular Disease Prevention in Clinical Practice (constituted by representatives of nine societies and by invited experts). Eur Heart J. Jul 2012;33(13):1635-1701.

9. Perk J, De Backer G, Gohlke H, et al. European Guidelines on cardiovascular disease prevention in clinical practice (version 2012). The Fifth Joint Task Force of the European Society of Cardiology and Other Societies on Cardiovascular Disease Prevention in Clinical Practice (constituted by representatives of nine societies and by invited experts). Eur Heart J. Jul 2012;33(13):1635-1701.

10. Mosca L, Benjamin EJ, Berra K, et al. Effectiveness-based guidelines for the prevention of cardiovascular disease in women--2011 update: a guideline from the american heart association. Circulation. Mar 22 2011;123(11):1243-1262.

11. Schenck-Gustafsson K, Rees M. Cardiology for gynecologists-A minireview. Maturitas. May 312013.

12. Bellamy L, Casas JP, Hingorani AD, Williams DJ. Pre-eclampsia and risk of cardiovascular disease and cancer in later life: systematic review and metaanalysis. BMJ. Nov 10 2007;335(7627):974.

13. Shaw LJ, Bairey Merz CN, Azziz R, et al. Postmenopausal women with a history of irregular menses and elevated androgen measurements at high risk for worsening cardiovascular event-free survival: results from the National Institutes of Health--National Heart, Lung, and Blood Institute sponsored Women's Ischemia Syndrome Evaluation. The Journal of clinical endocrinology and metabolism. Apr 2008;93(4):1276-1284.

14. Brown MC, Best KE, Pearce MS, Waugh J, Robson SC, Bell R. Cardiovascular disease risk in women with pre-eclampsia: systematic review and meta-analysis. Eur J Epidemiol. Jan 2013;28(1):1-19. 
15. McDonald SD, Malinowski A, Zhou Q, Yusuf S, Devereaux PJ. Cardiovascular sequelae of preeclampsia/eclampsia: a systematic review and meta-analyses. Am Heart J. Nov 2008;156(5):918-930.

16. Fraser A, Nelson SM, Macdonald-Wallis C, et al. Associations of pregnancy complications with calculated cardiovascular disease risk and cardiovascular risk factors in middle age: the Avon Longitudinal Study of Parents and Children. Circulation. Mar 20 2012;125(11):1367-1380.

17. Jessup M, Abraham WT, Casey DE, et al. 2009 focused update: ACCF/AHA Guidelines for the Diagnosis and Management of Heart Failure in Adults: a report of the American College of Cardiology Foundation/American Heart Association Task Force on Practice Guidelines: developed in collaboration with the International Society for Heart and Lung Transplantation. Circulation. Apr 14 2009;119(14):1977-2016.

18. Ammar KA, Jacobsen SJ, Mahoney DW, et al. Prevalence and prognostic significance of heart failure stages: application of the American College of Cardiology/American Heart Association heart failure staging criteria in the community. Circulation. Mar 27 2007;115(12):1563-1570.

19. Desvigne-Nickens P. Heart Failure Prevention Is the Best Option To Stem High Costs and Disease Burden Research for More Effective Heart Failure Treatment Is Needed. Circulation: Cardiovascular Quality and Outcomes. 2011;4(2):143-145.

20. Hill JA, Olson EN. Cardiac plasticity. N Engl J Med. Mar 27 2008;358(13):13701380.

21. Gaasch WH, Zile MR. Left ventricular structural remodeling in health and disease: with special emphasis on volume, mass, and geometry. J Am Coll Cardiol. Oct 18 2011;58(17):1733-1740.

22. Cohn JN, Ferrari R, Sharpe N. Cardiac remodeling--concepts and clinical implications: a consensus paper from an international forum on cardiac remodeling. Behalf of an International Forum on Cardiac Remodeling. J Am Coll Cardiol. Mar 1 2000;35(3):569-582.

23. Ruwhof $C$, van der Laarse A. Mechanical stress-induced cardiac hypertrophy: mechanisms and signal transduction pathways. Cardiovascular research. Jul 2000;47(1):23-37.

24. Perrino C, Naga Prasad SV, Mao L, et al. Intermittent pressure overload triggers hypertrophy-independent cardiac dysfunction and vascular rarefaction. J Clin Invest. Jun 2006;116(6):1547-1560.

25. Calderone A, Takahashi N, Izzo NJ, Jr., Thaik CM, Colucci WS. Pressure- and volume-induced left ventricular hypertrophies are associated with distinct myocyte phenotypes and differential induction of peptide growth factor mRNAs. Circulation. Nov 1 1995;92(9):2385-2390.

26. Kehat I, Molkentin JD. Molecular pathways underlying cardiac remodeling during pathophysiological stimulation. Circulation. Dec 21 2010;122(25):2727-2735.

27. Dorn GW, 2nd, Robbins J, Sugden PH. Phenotyping hypertrophy: eschew obfuscation. Circulation research. Jun 13 2003;92(11):1171-1175.

28. Opie LH, Commerford PJ, Gersh BJ, Pfeffer MA. Controversies in ventricular remodelling. Lancet. Jan 28 2006;367(9507):356-367. 
29. Dorn GW, 2nd. The fuzzy logic of physiological cardiac hypertrophy. Hypertension. May 2007;49(5):962-970.

30. Melchiorre K, Sutherland GR, Baltabaeva A, Liberati M, Thilaganathan B. Maternal cardiac dysfunction and remodeling in women with preeclampsia at term. Hypertension. Jan 2011;57(1):85-93.

31. Steegers EA, von Dadelszen P, Duvekot JJ, Pijnenborg R. Pre-eclampsia. Lancet. Aug 21 2010;376(9741):631-644.

32. Roberts JM, Gammill HS. Preeclampsia: recent insights. Hypertension. Dec 2005;46(6):1243-1249.

33. Roberts JM, Pearson GD, Cutler JA, Lindheimer MD, National Heart L, Blood I. Summary of the NHLBI Working Group on Research on Hypertension During Pregnancy. Hypertens Pregnancy. 2003;22(2):109-127.

34. von Dadelszen P, Magee LA, Roberts JM. Subclassification of preeclampsia. Hypertens Pregnancy. 2003;22(2):143-148.

35. Bodnar LM, Catov JM, Klebanoff MA, Ness RB, Roberts JM. Prepregnancy body mass index and the occurrence of severe hypertensive disorders of pregnancy. Epidemiology. Mar 2007;18(2):234-239.

36. Stekkinger E, Zandstra M, Peeters LL, Spaanderman ME. Early-onset preeclampsia and the prevalence of postpartum metabolic syndrome. Obstet Gynecol. Nov 2009;114(5):1076-1084.

37. Duckitt K, Harrington D. Risk factors for pre-eclampsia at antenatal booking: systematic review of controlled studies. BMJ. Mar 12 2005;330(7491):565.

38. Duvekot JJ, Cheriex EC, Pieters FA, Menheere PP, Peeters LH. Early pregnancy changes in hemodynamics and volume homeostasis are consecutive adjustments triggered by a primary fall in systemic vascular tone. Am J Obstet Gynecol. Dec 1993;169(6):1382-1392.

39. Hibbard JU, Shroff SG, Lang RM. Cardiovascular changes in preeclampsia. Semin Nephrol. Nov 2004;24(6):580-587.

40. Campos O. Doppler Echocardiography During Pregnancy: Physiological and Abnormal Findings. Echocardiography. Mar 1996;13(2):135-146.

41. Lang RM, Pridjian G, Feldman $T$, Neumann A, Lindheimer M, Borow KM. Left ventricular mechanics in preeclampsia. Am Heart J. Jun 1991;121(6 Pt 1):17681775.

42. Borghi C, Esposti DD, Immordino V, et al. Relationship of systemic hemodynamics, left ventricular structure and function, and plasma natriuretic peptide concentrations during pregnancy complicated by preeclampsia. Am J Obstet Gynecol. Jul 2000;183(1):140-147.

43. Rafik Hamad R, Larsson A, Pernow J, Bremme K, Eriksson MJ. Assessment of left ventricular structure and function in preeclampsia by echocardiography and cardiovascular biomarkers. J Hypertens. Nov 2009;27(11):2257-2264.

44. Goldberg LR, Jessup M. Stage B heart failure: management of asymptomatic left ventricular systolic dysfunction. Circulation. Jun 20 2006;113(24):2851-2860.

45. Lauer MS, Anderson KM, Levy D. Influence of contemporary versus 30-year blood pressure levels on left ventricular mass and geometry: the Framingham Heart Study. J Am Coll Cardiol. Nov 1 1991;18(5):1287-1294. 

Part 1

Cardiac adaption after preeclampsia 



\section{Chapter 2:}

Cardiac adaptation to the next pregnancy in formerly preeclamptic women bound to develop recurrent preeclampsia

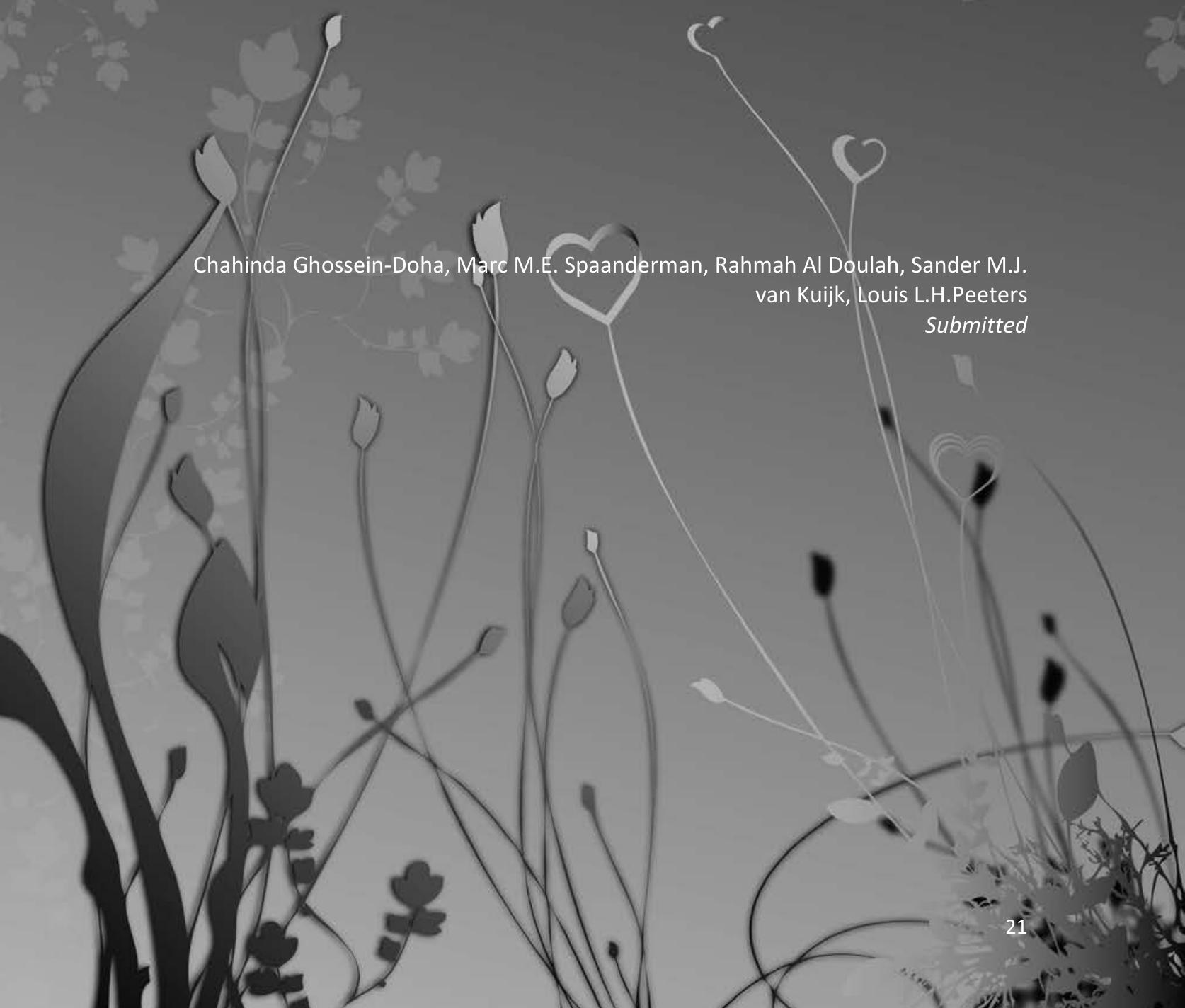




\begin{abstract}
Objective: Left-ventricular remodeling in preeclampsia $(\mathrm{PE})$ is concentric rather than eccentric. After early-onset PE, it tends to persist. The objective of this observational study was to determine, whether pre-pregnancy cardiac geometry and function along with first and early second trimester cardiac adaptation to the next pregnancy in former early-onset PE patients differ between former patients who do (RECUR) and do not (NONRECUR) develop recurrent PE later on in that pregnancy.
\end{abstract}

Study design: In 51 women with a history of early-onset PE, we performed serial cardiac ultrasound and recorded automated blood pressure/heart rate before pregnancy and again three times early in the next pregnancy. From the hospital records, we retrieved the information on pregnancy outcome. We compared the intergroup differences in cardiac indices using the independent samples-t-test, and the inter-group differences in pre-pregnant echocardiographic indices and the subsequent pregnancy-induced cardiac adaptive response by repeated measures analysis of variance (ANOVA).

Results: PE recurred in 14/51 (27\%) women. Preconceptionally, RECUR differed from NONRECUR by a higher resting heart rate (71 vs. 64 beats $\min ^{-1}, p<0.05$ ), a lower stroke volume (68 vs. $77 \mathrm{~mL}, \mathrm{p}<0.05$ ), and a lower left-ventricular mass index (28 versus $32 \mathrm{~g}$. $\left.\mathrm{m}^{-2.7}, \mathrm{p}<0.05\right)$. Despite these pre-pregnancy circulatory differences, the initial pregnancy-induced pattern of cardiac adaptive response did not differ appreciably between the two subgroups.

Conclusion: Even though pre-pregnant cardiac geometry and function differed between RECUR and NONRECUR, the pattern of cardiac adaptation to the next pregnancy was comparable. 


\section{Introduction}

Preeclampsia (PE) complicates 2 to $5 \%$ of all pregnancies ${ }^{1}$ and is defined by clinical features in the absence of a clear insight in its cause. PE appears to be the common endpoint of various disorders with different pathogenesis eventually merging into the classical clinical syndrome. Therefore, exploration of the preclinical phase is expected to open new avenues in unravelling the pathogenesis of PE.

In the preclinical phase of PE, the circulation may be "hypodynamic", that is to say, basal metabolic demands are fulfilled by a circulatory function characterized by a relatively low cardiac output (CO) with a correspondingly higher total peripheral vascular resistance (TPVR). Conversely, in other cases of PE the preclinical circulation may be "hyperdynamic" which indicates that basal metabolic demands are achieved with a circulation that combines a relatively high $\mathrm{CO}$ with a proportionately lower TPVR. In most cases, early-onset PE is preceded by a hypodynamic-, and late-onset PE by a hyperdynamic circulation. ${ }^{2,3}$

The risk of recurrent PE in the next pregnancy varies from 6-50\% amongst different PEsubgroups, the highest recurrence rate being observed after early-onset PE. ${ }^{1}$ In the latent phase of early-onset PE, plasma volume (PV) is often subnormal ${ }^{4}$ and accompanied by sympathetic dominance in the autonomic control of the circulation. $^{5,6,7}$ In normal pregnancy left-ventricular (LV) remodeling is eccentric ${ }^{8,9}$ indicating that LV mass increases in concert with LV widening. The latter cardiac adaptive response is often paralleled by a higher cardiac preload, reflected in adequate pregnancy-induced PV expansion.

In the clinical phase of PE, left-ventricular remodeling is concentric, ${ }^{8,9}$ indicating that LV mass increases without concomitant ventricular widening. This abnormal adaptive response is often paralleled by a subnormal, poorly expanding PV, a raised cardiac afterload and cardiovascular sympathetic over-activity. This aberrant cardiac geometry and function triggered by PE may persist postpartum, especially after early-onset PE. ${ }^{10}$ We postulate that incomplete resolution of the concentrically remodeled heart after early-onset PE hampers normal cardiac adaptation to a next pregnancy, and therefore, increases the risk of developing recurrent PE.

This study was designed to test the hypothesis that women with a history of earlyonset $\mathrm{PE}$ developing recurrent $\mathrm{PE}$ in their next pregnancy differ from their counterparts with a normal next pregnancy by 1) abnormal pre-pregnant echocardiographic indices, and/or 2) an abnormal pattern of initial cardiac adaptive response to that next pregnancy. To this end, we compared the pre-pregnant echocardiographic indices and the initial pregnancy-induced pattern in cardiac adaptive response between women with a history of early-onset PE, who did and did not develop recurrent $\mathrm{PE}$.

\section{Materials and Methods}

\section{Study population}

The Maastricht University Medical Centre (MUMC) Medical Ethics Committee (May 2010, MEC 10-4-049) approved this longitudinal observational cohort study. At the 
MUMC, all women who have a pregnancy complicated by a hypertensive disorder are routinely screened for underlying cardiovascular disorders at least 6 months postpartum. In case of breastfeeding, women are only scheduled for screening at least 4 weeks after weaning. Patients are asked to discontinue the use of oral contraceptives at least one month before screening. The cardiovascular screening is repeated at 3 consecutive time-points in the first half of the next pregnancy (12, 16 and 20 weeks). Antihypertensive drugs are discontinued 2 weeks prior to the first assessment (prepregnant). All former patients enrolled in the present study completed their next pregnancy between March 2002 and December 2011. We diagnosed PE according to the criteria specified in 2000 by the National Working Group on High Blood Pressure in Pregnancy, namely when de-novo hypertension (blood pressure $>140 / 90 \mathrm{mmHg}$ ) had developed accompanied by de novo proteinuria (> $300 \mathrm{mg} \cdot 24 \mathrm{~h}^{-1}$ or $>30 \mathrm{~g} \cdot \mathrm{mol}^{-1}$ creatinine) after the $20^{\text {th }}$ week, or when de novo proteinuria had developed after the $20^{\text {th }}$ week in a woman with pre-existing hypertension. ${ }^{11}$ Early-onset PE is defined as PE requiring pregnancy termination before the $34^{\text {th }}$ completed week. Women with an elevated blood pressure detected after the $20^{\text {th }}$ week without de novo proteinuria are classified as having gestational hypertension. ${ }^{11}$ Birth weight below the $5^{\text {th }}$ centile based on the Dutch birth weight reference curves is considered to indicate small-forgestational-age (SGA). ${ }^{12}$

Our dataset contained 84 primiparous women with a history of early-onset PE eligible for inclusion. To secure the set-up of two as homogeneous as possible subgroups, we only included former patients who either had developed pure recurrent PE (RECUR) or no hypertensive pregnancy disorder whatsoever (NONRECUR). Consequently, we excluded women who had developed gestational hypertension (PIH, n=17), the HELLP syndrome $(n=2)$, and/or who had given birth to an SGA infant in the absence of symptoms of a hypertensive disorder $(n=3)$. Eleven women were excluded because of not conceiving anymore or because they were lost to follow up. Fourteen of the remaining 51 women were allocated to RECUR and 37 to NONRECUR.

\section{Measurements}

At least 10 hours before each measurement session, participants refrained from eating, from drinking caffeine- or alcohol-containing beverages and, if applicable, from smoking. All measurement sessions started at 8:00 am in a temperature-controlled room $( \pm 24 \stackrel{\circ}{\circ}$ ) with external disturbances kept to a minimum.

Throughout the measurements, participants were either lying on their back (prepregnant) or in left-lateral tilt position with a rolled blanket placed under their left flank (during pregnancy). After an acclimatization period of 30 minutes, we recorded arterial blood pressure at 3-minutes intervals for a period of 30 minutes by using a semi-automatic oscillometric device (Dinamap Vital Signs Monitor 1846; Critikon, Tampa, Florida, USA). BMI $\left(\mathrm{kg} \cdot \mathrm{m}^{-2}\right)$ was calculated by dividing body weight $(\mathrm{kg})$ by the squared height in meters $\left(\mathrm{m}^{2}\right)$. An estimate for total fat mass $(\mathrm{kg})$ was obtained by multiplying height $(\mathrm{m})$ with $([1.2 \cdot \mathrm{BMI}]+[0.23 \cdot$ age $]-5.4) .{ }^{13}$ Lean Body Mass (LBM, kg) was obtained by subtracting total fat mass from bodyweight. 
We calculated body surfacearea (BSA) as follows: BSA $\left(\mathrm{m}^{2}\right)=0.007184 \cdot([$ height $(\mathrm{cm})]$ $\left.0.725 \cdot[\text { weight }(\mathrm{kg})]^{0.425}\right){ }^{14}$

We measured plasma volume (PV, $\mathrm{mL} \cdot[\mathrm{kg} \text { lean body mass }]^{-1}$ ) before pregnancy using the indicator dilution technique ${ }^{15,16}$ with $\mathrm{I}^{125}$-labelled albumin. To measure PV during pregnancy we used the $10 \%$ dextran-70 solution as specified previously. ${ }^{17} \mathrm{We}$ considered a PV below $48 \mathrm{~mL} \cdot \mathrm{kg}^{-1} \mathrm{LBM}$, corresponding with 2 SD below the mean PV of a control group, as subnormal. ${ }^{18,19}$

Echocardiography. After the PV measurement, we assessed cardiac structure and function using cardiac ultrasound. To this end, we invited the participant to lay on her back in semi-left lateral tilt position and performed the measurement using a Philips IE 33 system for phased-array echocardiography and Doppler (Philips Medical System, Best, The Netherlands). We stored all data on disk and performed all calculations offline using specific software (Excelera, Philips, the Netherlands). The twodimensional M-mode and Doppler echocardiography were performed according to the guidelines of the American Society of Echocardiography to assess relevant cardiac indices $^{20}$. Using the M-mode in the parasternal long-axis view, we measured left ventricular end-diastolic and end-systolic diameters (LVEDd, LVESd, $\mathrm{mm}$ ), end-diastolic wall thickness of the inter-ventricular septum (IVST, mm), and posterior wall thickness (PWT, mm). LVM was calculated using the Devereux formula as follows: LVM (g) = 1.04 $\left[(\text { LVEDd + PWT + IVST })^{3}-\right.$ LVEDd $\left.\left.^{3}\right)-14\right] .{ }^{21}$ LVM is indexed for height (in $\mathrm{m}^{2.7}$ ) as follows: $L V M i=\left(L V M \cdot m^{-2.7}\right){ }^{22}$ Finally, we calculated the relative wall thickness (RWT) as follows: RWT $=($ IVST + PWT $) \cdot$ LVEDd $^{-1}$.

Systolic function. Left-ventricular systolic function was evaluated as follows: heart rate (HR, beats $\cdot \mathrm{min}^{-1}$ ) was obtained by taking the reciprocal of the mean of 5 consecutive RR-intervals ( $\mathrm{sec}$ ) on the electrocardiogram (ECG) multiplied by 60 . The mean aortic Velocity Time Integral (VTI) was obtained from the average of the outer edge tracings of 5 consecutive continuous wave (CW) Doppler registrations of the flow across the aortic valve. Stroke volume (SV, $\mathrm{mL}$ ) was calculated by multiplying the VTI with the cross-sectional area measured at the level of the aortic annulus in the parasternal long-axis view, and $\mathrm{CO}\left(\mathrm{L} \cdot \mathrm{min}^{-1}\right)$ by multiplying SV with HR. Cardiac index $(\mathrm{Cl})$ was obtained by the formula: $\mathrm{Cl}=\mathrm{CO} \cdot \mathrm{BSA}^{-1}$.

Diastolic function. The diastolic function was derived from the transmitral flow pattern obtained by pulsed-wave (PW) Doppler echocardiography in the apical 4-chamber view. The PW Doppler sample volume $(5 \mathrm{~mm}$ ) was carefully positioned at the tip of the mitral valve leaflets with sweep rate set at $50 \mathrm{~mm} \cdot \mathrm{s}^{-1}$. We defined the E/A ratio as the ratio of peak mitral flow velocity in early diastole (E) and that during atrial contraction (A). Doppler-derived indices were averaged over 5 consecutive cardiac cycles.

Total Peripheral Vascular Resistance (TPVR in dynes $\cdot \mathrm{sec} \cdot \mathrm{cm}^{-5}$ ) was obtained by taking the ratio of mean arterial pressure (MAP, $\mathrm{mmHg}$ ) and $\mathrm{CO}$, multiplied by 80 .

Statistical analysis. Demography and obstetrical history are presented as mean, median or proportion. Non-normally distributed data was analyzed using the Mann Whitney $U$ test and dichotomous data with the $X^{2}$ test. We compared the cardiac variables between RECUR and NONRECUR using the independent samples-t-test. Changes induced by pregnancy in both subgroups were evaluated using the paired 
samples-t-test on the difference between the pre-pregnancy and 20 weeks' measurement. To test for differences in cardiac adaptive response to pregnancy, both in between and within the two study groups, we used repeated measures analysis of variance (ANOVA) on the 4 serially-measured variables. For the within subjects difference we corrected for multiple testing by Bonferroni. This study always involved at least two measurements per participant, the pre-pregnancy data point always being available except for one woman. Of the 51 participants, 33 had 4 data points (65\%), 14 had 3 data points (27\%) and 4 had 2 data points (8\%). We imputed missing values by regression imputation, as the use of participants with a complete dataset only (65\%) would result in loss of precision. ${ }^{23}$ All analyses were performed using SPSS version 20.0. A p-value below 0.05 was considered statistically significant.

\section{Results}

Table 1 lists the demography and clinical characteristics of both study groups. Women in the RECUR group were younger and had more often a subnormal pre-pregnant PV than their counterparts in the NONRECUR group. In their next pregnancy, RECUR women also differed from NONRECUR by a \pm 3 weeks earlier delivery, giving birth to an infant with a lower birth weight not only due to more severe prematurity but also because of a higher incidence of SGA births.

Table 1: Demography and obstetrical history of both subgroups. Data are presented as mean ( \pm SD), or - in case of skewed data distribution - as median (with IQR) unless otherwise stated. Dichotomous data are presented as $n,(\%)$.

\begin{tabular}{lcc}
\hline & $\begin{array}{c}\text { NONRECUR } \\
\mathrm{n}=37\end{array}$ & $\begin{array}{c}\text { RECUR } \\
\mathrm{n}=14\end{array}$ \\
\hline Maternal age at pre-pregnancy measurement (y) & $30(29-33)$ & $27(24-31)^{\star}$ \\
BMI $\left(\mathrm{kg} \cdot \mathrm{m}^{-2}\right)$ & $27 \pm 6$ & $25 \pm 5$ \\
Height (cm) & $170 \pm 6$ & $166 \pm 6$ \\
Chronic hypertension (n, \%) & $14(38 \%)$ & $7(50 \%)$ \\
Inter-pregnancy interval (y, median/range) & $3(1-13)$ & $3(1-6)$ \\
Index pregnancy & & \\
Maternal age (y) & $29(27-32)$ & $27(23-29)^{\star}$ \\
Gestational age at birth (weeks) & $31.1(28.9-32.2)$ & $29.8(27.0-30.1)$ \\
Birth weight (g) & $1150(753-1718)$ & $970(700-1370)$ \\
SGA (n, \%) & $8(23 \%)$ & $4(29 \%)$ \\
Next pregnancy & & $28(25-33)$ \\
Maternal age (y) & $33(30-35)$ & $12 / 14(86 \%)^{\star}$ \\
Subnormal PV & $22 / 37(59 \%)$ & $6 / 14(43 \%)$ \\
Early-onset preeclampsia (n, \%) & - & $37.1(32.2-38.2)^{\star}$ \\
Gestational age at birth (weeks) & $38.7(38.0-40.0)$ & $2277(1599-3080)^{\star}$ \\
Birth weight (g) & $3360(3120-3718)$ & $3(21 \%)$ \\
SGA (n, \%) & - &
\end{tabular}

PE: preeclampsia;

${ }^{*} \mathrm{p}<0.05$ compared with NONRECUR 
Tables 2 and 3 indicate that before pregnancy, RECUR differed from NONRECUR by a $\approx$ $10 \%$ higher HR, a correspondingly lower SV and an approx. 12\% lower LVMi. At 20 weeks pregnancy, RECUR differed from NONRECUR by higher BPdias, higher MAP, and lower E/A ratio. Despite these initial differences, pregnancy not only induced a comparable pattern of change in cardiac structure in both study groups, but also a comparable relative and absolute rise in $\mathrm{CO}, \mathrm{Cl}, \mathrm{BPs}$ and TPVR.

Fig 1 illustrates that the pattern of pregnancy-induced changes in LVMi $(p=0.89), E / A$ ratio, HR and SV was similar in both study groups, even though pre-pregnant LVMi in RECUR was persistently $\approx 12 \%$ lower than in NONRECUR (Fig.1a, p<0.05). The prepregnant E/A ratio, and its change in response to pregnancy, were comparable in the 2 subgroups, although this ratio had become significantly lower in RECUR by 20 weeks (Fig. 1b, table 2). Figures $1 \mathrm{c}$ and $1 \mathrm{~d}$ show that RECUR differs from NONRECUR by a consistently higher pre-pregnant HR with correspondingly lower SV. These differences persisted in the first half of pregnancy and were accompanied by similar absolute changes in both study groups. Of the 51 former patients, 34 had a subnormal PV (table 1 ), with 12 (35\%) developing recurrent PE. In contrast, only 2 of the 17 former patients with a normal PV (11\%) had developed recurrent PE. 
Table 2: Hemodynamic indices before the second pregnancy and again at 20 weeks of gestation. Groups are compared with the independent samples t-test. The within group adaptation is analysed with the paired t-test. Data are presented as mean \pm SD.

\begin{tabular}{|c|c|c|c|c|}
\hline & & $\begin{array}{c}\begin{array}{c}\text { NONRECUR } \\
\mathrm{N}=37\end{array} \\
\end{array}$ & $\begin{array}{c}\text { RECUR } \\
\mathrm{N}=14\end{array}$ & $\mathrm{p}$-value \\
\hline \multicolumn{5}{|c|}{ Hemodynamic parameters } \\
\hline \multirow[t]{3}{*}{ BPsys (mmHg) } & pre-gravid & $120 \pm 14$ & $125 \pm 17$ & 0.23 \\
\hline & 20 weeks & $109 \pm 12^{\#}$ & $114 \pm 11^{\#}$ & 0.16 \\
\hline & $\Delta$ & $-11 \pm 13$ & $-12 \pm 18$ & 0.89 \\
\hline \multirow[t]{3}{*}{ BPdias (mmHg) } & pre-gravid & $74 \pm 9$ & $81 \pm 10$ & 0.06 \\
\hline & 20 weeks & $65 \pm 7^{\#}$ & $70 \pm 8^{\#}$ & $<0.05$ \\
\hline & $\Delta$ & $-9 \pm 9$ & $-11 \pm 9$ & 0.50 \\
\hline \multirow[t]{3}{*}{$\operatorname{MAP}(\mathrm{mmHg})$} & pre-gravid & $92 \pm 11$ & $96 \pm 12$ & 0.29 \\
\hline & 20 weeks & $81 \pm 9 \#$ & $86 \pm 7^{\#}$ & $<0.05$ \\
\hline & $\Delta$ & $-11 \pm 10$ & $-10 \pm 9$ & 0.65 \\
\hline \multirow[t]{3}{*}{ HR (beats $\cdot \min ^{-1}$ ) } & pre-gravid & $64 \pm 10$ & $71 \pm 10$ & $<0.05$ \\
\hline & 20 weeks & $72 \pm 9^{\#}$ & $76 \pm 8$ & 0.19 \\
\hline & $\Delta$ & $8 \pm 8$ & $5 \pm 13$ & 0.31 \\
\hline \multirow[t]{3}{*}{ SV (ml) } & pre-gravid & $77 \pm 14$ & $68 \pm 10$ & $<0.05$ \\
\hline & 20 weeks & $81 \pm 17$ & $74 \pm 19$ & 0.23 \\
\hline & $\Delta$ & $4 \pm 15$ & $6 \pm 19$ & 0.66 \\
\hline \multirow[t]{3}{*}{$\mathrm{CO}\left(L \cdot \min ^{-1}\right)$} & pre-gravid & $5.1 \pm 1.0$ & $5.0 \pm 0.8$ & 0.64 \\
\hline & 20 weeks & $5.8 \pm 1.1^{\#}$ & $5.8 \pm 1.1^{\#}$ & 0.95 \\
\hline & $\Delta$ & $0.7 \pm 1.1$ & $0.8 \pm 0.9$ & 0.64 \\
\hline \multirow[t]{3}{*}{$\mathrm{Cl}\left(\mathrm{L} \cdot \mathrm{min}^{-1} \cdot \mathrm{m}^{-2}\right)$} & pre-gravid & $2.7 \pm 0.4$ & $2.7 \pm 0.5$ & 0.94 \\
\hline & 20 weeks & $3.1 \pm 0.5^{\#}$ & $3.2 \pm 0.4^{\#}$ & 0.44 \\
\hline & $\Delta$ & $0.3 \pm 0.5$ & $0.5 \pm 0.5$ & 0.41 \\
\hline \multirow[t]{3}{*}{$\mathrm{EF}(\%)$} & pre-gravid & $64 \pm 4$ & $64 \pm 4$ & 0.67 \\
\hline & 20 weeks & $62 \pm 3$ & $62 \pm 4$ & 0.51 \\
\hline & $\Delta$ & $-1 \pm 5$ & $-2 \pm 6$ & 0.44 \\
\hline \multirow[t]{3}{*}{ TPVR (dynes.s.cm-5) } & pre-gravid & $1511 \pm 260$ & $1622 \pm 252$ & 0.18 \\
\hline & 20 weeks & $1132 \pm 162^{\#}$ & $1141 \pm 208^{\#}$ & 0.89 \\
\hline & $\Delta$ & $-370 \pm 295$ & $-490 \pm 347$ & 0.22 \\
\hline \multirow[t]{3}{*}{ E/A ratio } & pre-gravid & $1.6 \pm 0.4$ & $1.4 \pm 0.3$ & 0.09 \\
\hline & 20 weeks & $1.6 \pm 0.3$ & $1.4 \pm 0.3$ & $<0.05$ \\
\hline & $\Delta$ & $0.0 \pm 0.4$ & $0.0 \pm 0.3$ & 0.94 \\
\hline \multirow[t]{3}{*}{ PV (ml) } & pre-gravid & $2429 \pm 393$ & $2308 \pm 248$ & 0.29 \\
\hline & 20 weeks & $2901 \pm 501^{\#}$ & $2690 \pm 536^{\#}$ & 0.19 \\
\hline & $\Delta$ & $471 \pm 690$ & $382 \pm 551$ & 0.67 \\
\hline \multirow[t]{3}{*}{ PVindex $\left(\mathrm{ml} \cdot \mathrm{kgLBM}^{-1}\right)$} & pre-gravid & $46.0 \pm 9.0$ & $43.5 \pm 5.5$ & 0.32 \\
\hline & 20 weeks & $54.8 \pm 9.3^{\#}$ & $52.9 \pm 8.2^{\#}$ & 0.51 \\
\hline & $\Delta$ & $8.8 \pm 12.1$ & $9.5 \pm 6.6$ & 0.84 \\
\hline
\end{tabular}

BPsys: Systolic Blood Pressure; BPdias: Diastolic Blood Pressure; MAP: Mean Arterial Pressure; HR: Heart Rate; SV: Stroke Volume; CO: Cardiac Output; Cl: Cardiac Index; EF: ejection fraction; EA ratio: ratio of peak mitral flow velocity in early diastole (E) and that during atrial contraction (A); PV: plasma volume; TPVR: Total Peripheral Vascular Resistance. ${ }^{*} p<0.05$ compared with NONRECUR $\# p<0.05$ compared with pre-pregnancy value 
Table 3: Cardiac indices prior to the second pregnancy and again at 20 weeks of gestation. Groups are compared with the independent samples t-test. The within group adaptation is analyzed with the paired-t-test. Data are presented as mean \pm SD.

\begin{tabular}{|c|c|c|c|c|}
\hline & & $\begin{array}{c}\text { NONRECUR } \\
\mathrm{N}=37\end{array}$ & $\begin{array}{c}\text { RECUR } \\
\mathrm{N}=14\end{array}$ & $\mathrm{p}$-value \\
\hline \multicolumn{5}{|c|}{ Cardiac Geometry } \\
\hline \multirow[t]{3}{*}{ LVM (g) } & pre-gravid & $148 \pm 29$ & $128 \pm 26^{*}$ & $<0.05$ \\
\hline & 20 weeks & $155 \pm 27$ & $145 \pm 27$ & 0.22 \\
\hline & $\Delta$ & $7 \pm 20$ & $12 \pm 36$ & 0.67 \\
\hline \multirow[t]{3}{*}{ LVMI (g·m-2.7) } & pre-gravid & $32 \pm 6$ & $28 \pm 5^{*}$ & $<0.05$ \\
\hline & 20 weeks & $33 \pm 6$ & $30 \pm 6$ & 0.07 \\
\hline & $\Delta$ & $1 \pm 5$ & $1 \pm 7$ & 0.90 \\
\hline \multirow[t]{3}{*}{ IVST (mm) } & pre-gravid & $7.7 \pm 0.8$ & $7.2 \pm 0.6$ & 0.13 \\
\hline & 20 weeks & $7.6 \pm 1.0$ & $7.6 \pm 1.0$ & 0.98 \\
\hline & $\Delta$ & $-0.04 \pm 1.0$ & $0.4 \pm 0.8$ & 0.13 \\
\hline \multirow[t]{3}{*}{ PWT (mm) } & pre-gravid & $7.7 \pm 0.7$ & $7.8 \pm 0.8$ & 0.59 \\
\hline & 20 weeks & $7.9 \pm 0.7$ & $7.8 \pm 0.8$ & 0.90 \\
\hline & $\Delta$ & $0.1 \pm 0.9$ & $0.04 \pm 0.7$ & 0.70 \\
\hline \multirow[t]{3}{*}{ RWT (fraction) } & pre-gravid & $0.33 \pm 0.04$ & $0.34 \pm 0.03$ & 0.52 \\
\hline & 20 weeks & $0.32 \pm 0.03$ & $0.31 \pm 0.03$ & 0.36 \\
\hline & $\Delta$ & $-0.01 \pm 0.04$ & $-0.03 \pm 0.04$ & 0.21 \\
\hline \multicolumn{5}{|c|}{ Cardiac dimensions } \\
\hline \multirow[t]{3}{*}{ LVEDd (mm) } & pre-gravid & $47 \pm 4$ & $46 \pm 4$ & 0.19 \\
\hline & 20 weeks & $49 \pm 3^{\#}$ & $48 \pm 3^{\#}$ & 0.36 \\
\hline & $\Delta$ & $2 \pm 4$ & $2 \pm 4$ & 0.62 \\
\hline \multirow[t]{3}{*}{ LVESd (mm) } & pre-gravid & $31 \pm 3$ & $29 \pm 3$ & 0.06 \\
\hline & 20 weeks & $32 \pm 2^{\#}$ & $31 \pm 2^{\#}$ & 0.09 \\
\hline & $\Delta$ & $2 \pm 2$ & $2 \pm 3$ & 0.57 \\
\hline \multirow[t]{3}{*}{ LAD (mm) } & pre-gravid & $36 \pm 4$ & $34 \pm 4$ & 0.18 \\
\hline & 20 weeks & $37 \pm 4^{\#}$ & $36 \pm 4$ & 0.11 \\
\hline & $\Delta$ & $2 \pm 5$ & $2 \pm 5$ & 0.94 \\
\hline
\end{tabular}

LVMI: left ventricular mass; LVMI: Left Ventricular Mass index; IVST: Intra-Ventricular Septum Thickness;

PWT: Posterior Wall Thickness; RWT: Relative Wall Thickness; LVEDd: Left Ventricular End Diastolic Diameter; LVESd: Left Ventricular End-Systolic Diameter; LAD: Left Atrial Diameter;

* $p<0.05$ compared with NONRECUR

$\# p<0.05$ compared with Pre-gravid value 

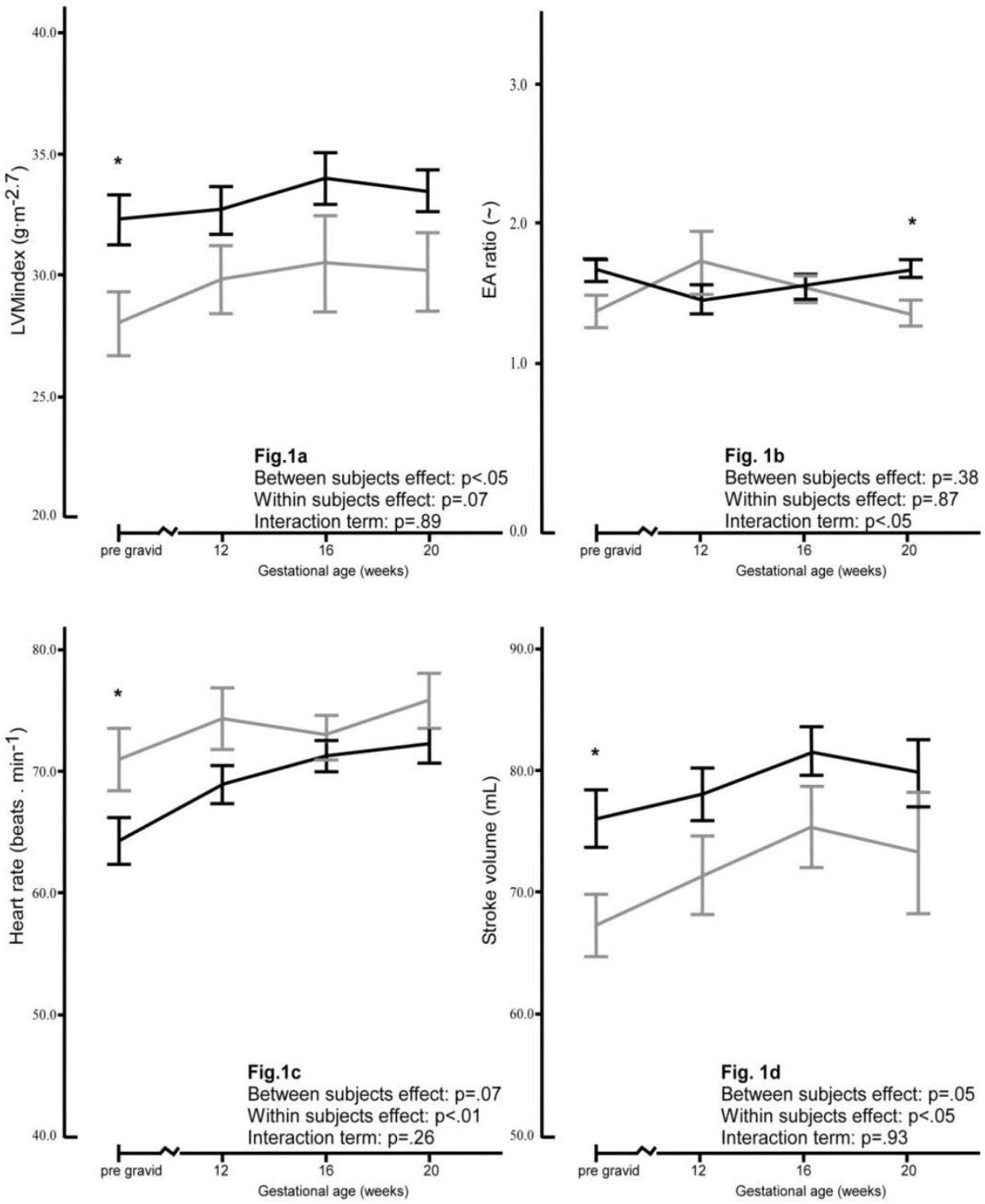

Figure 1: Mean \pm SEM of left ventricular mass index (LVMi), EA ratio, heart rate and stroke volume in pre-gravid state and at 12, 16 and 20 weeks of gestation in women with a history of early-onset PE who did (grey) and did not (black) develop a recurrent PE.

The between-subject effect tests whether the patterns in both groups with pregnancy differ significantly; the within-subject effect tests whether the patterns in each of the 2 groups changes consistently over time.

${ }^{*} p<0.05$ compared to Non-recurrent at time point 


\section{Comment}

Women with a history of early-onset PE developing recurrent PE in their next pregnancy differ in their post index-pregnancy evaluation from their counterparts not developing recurrent disease by a lower LVMi, lower SV, and higher resting HR. This suggests that their hearts are smaller with a correspondingly lower functional capacity. Although both subgroups seemed to respond to pregnancy with a comparable pattern of cardiac adaptation, some interesting functional differences were noted.

Although the pregnancy-induced \% rise in SV was larger in RECUR than in NONRECUR ( $9 \%$ vs. $5 \%$ ), the absolute SV at 20 weeks pregnancy was still $9 \%$ lower in RECUR than in NONRECUR. It appears that the pattern of cardiac adaptation to pregnancy of the presumably smaller heart in RECUR patients is similar to that in NONRECUR. Assuming comparable extra cardiac demands of pregnancy, RECUR patients are forced to raise HR to achieve a similar rise in CO. This suggests that the extra cardiac demands of pregnancy in RECUR are met by further increasing the already elevated sympathetic contribution to the autonomic control of the cardiac function. ${ }^{24}$

In the first trimester of human pregnancy, $C O$ increases by about $40 \%$ secondary to an initial fall in TPVR ${ }^{25}$ and rise in PV. In concert with these early hemodynamic changes, LVM and cardiac dimensions increase giving rise to the physiologic eccentric cardiac remodeling. ${ }^{26,27}$ This type of cardiac remodeling resembles that in athletes, and resolves in the first weeks postpartum. ${ }^{8}$ Pregnant women who develop PE also elicit a comparable drop in TPVR, although their sympathetic activity is higher and their absolute PV lower than in normotensive pregnancies. ${ }^{6,24}$ Under these conditions, the adaptive increase in LVM during the period of clinical disease does not seem to be paralleled by additional LV widening. It follows that the extra rise in LVM reflects concentric cardiac remodeling presumably secondary to the raised cardiac afterload during PE. ${ }^{28}$ This abnormal cardiac remodeling may persist for a prolonged period postpartum and may not resolve at all, particularly after early-onset PE. ${ }^{9,10}$

The difference in cardiac function between former patients who do and do not develop recurrent PE supports the concept that the former group of women has a relatively smaller and stiffer heart. To the best of our knowledge, this phenomenon has not been reported previously, although it seems to resemble to some extend the so-called "small-heart-syndrome", a disorder associated with the chronic fatigue syndrome. $^{29}$ There are no reports about the consequences of this disorder for pregnancy outcome. It is conceivable that the consistently lower SV along with the correspondingly higher HR indicates a lower cardiac functional capacity, that is to say, a reduced cardiac ability to raise SV in response to a rise in cardiac preload. Also E/A ratio seemed lower, although the difference only reached significance at 20 weeks pregnancy. When the heart of these women is exposed to more preload, as seen in the first half of their next pregnancy in response to the pregnancy-induced rise in PV, their relatively small heart appears to be stretched towards volumes in which the heart becomes stiffer. Theoretically the latter ought to be accompanied by a concomitant decline in E/A ratio. However, although the absolute values of the E/A ratio at 20 weeks pregnancy were significantly lower in RECUR, we did not discern a significantly lower E/A ratio at 20 weeks pregnancy relative to pre-pregnancy. The latter can at least in part be explained by the inflated type-2 error when evaluating a change with 
time in a ratio composed of 2 parameters with their own measurement error. Nevertheless, the higher HR reflects a larger basal sympathetic contribution to the autonomic control of the circulation ${ }^{24}$, presumably to compensate for the relatively low SV. In this line of reasoning, the reserves to raise CO in this condition are limited, which implies a higher chance in late pregnancy of lagging uteroplacental perfusion and thus of developing recurrent PE.

Obviously, our study has some limitations. The first drawback of our study is that PE developing in first- and subsequent pregnancies may have a different pathogenesis. A different pathogenesis of PE in $1^{\text {st }}$ and $2^{\text {nd }}$ pregnancies leads to scatter and inconsistent results in cohort studies. The latter effect represents the so-called "Simpsons paradox" ${ }^{30}$ which indicates that the study of a heterogeneous population may provide biased or counter-intuitive results, when that population is not divided into homogeneous subpopulations. Because of the latter, we decided to only include former patients with an as pure as possible clinical diagnosis of PE in RECUR and compare those with former patients without any hypertension or other milder form of the maternal placental syndrome in NONRECUR. Inherent to this approach is the issue of PE being defined based on clinical signs, which identifies a population which is clinically at high risk for complications, rather than a pathophysiological homogeneous population. ${ }^{31}$ Therefore, we decided to refrain from additional subgroup analysis, given the limited size and heterogeneity of our study population. A second limitation of our study is possibly the choice for imputing missing data. Omitting patients from the analysis because of one or more missing values would have led to loss of data and, with it, loss of statistical power. ${ }^{22}$ In addition; it could have led to biased results. However, as imputation does not guarantee unbiased estimates, we compared results obtained with and without data imputation. As the outcome of that calculation in both analyses pointed in the same direction, we are confident that the extent of missing values in our dataset had no appreciable effect on the results.

\section{Conclusion}

Based on the results of this study, we conclude that women with a history of earlyonset PE in their first pregnancy and recurrent PE in their next pregnancy differ from their counterparts with a normotensive next pregnancy by a lower pre-pregnancy LVMi, a lower SV and a higher HR. In spite of a similar pattern of change in cardiac geometry and function, absolute changes in response to pregnancy in RECUR seemed inadequate to meet the raised cardiac demands of pregnancy. In future studies on cardiac function and $\mathrm{PE}$, we recommend to evaluate pre-pregnant values as well as to stratify for the different subtypes of PE. 


\section{Details of ethics approval}

The study protocol was approved by the Maastricht University Medical Centre (MUMC) Medical Ethics Committee May 2010, MEC 10-4-049. The followed procedures were in conformity to 323 institutional guidelines and adhered to the principles of the Declaration of Helsinki and Title 45, U.S. Code of Federal Regulation, Part 46, Protection of Human Subjects, Revised November 13, 2001, effective December 13, 2001. 


\section{References}

1. Hernandez-Diaz S, Toh S, Cnattingius S. Risk of pre-eclampsia in first and subsequent pregnancies: prospective cohort study. BMJ. 2009;338:b2255.

2. Vatten LJ, Skjaerven R. Is pre-eclampsia more than one disease? $\mathrm{Br} \mathrm{J}$ Obstet Gynaecol. Apr 2004;111(4):298-302.

3. Valensise H, Vasapollo B, Gagliardi G, Novelli GP. Early and late preeclampsia: two different maternal hemodynamic states in the latent phase of the disease. Hypertension. Nov 2008;52(5):873-880.

4. Aardenburg R, Spaanderman ME, van Eijndhoven HW, de Leeuw PW, Peeters LL. A low plasma volume in formerly preeclamptic women predisposes to the recurrence of hypertensive complications in the next pregnancy. J Soc Gynecol Investig. Dec 2006;13(8):598-603.

5. Krabbendam I, Courtar DA, Janssen BJ, Aardenburg R, Peeters LL, Spaanderman ME. Blunted autonomic response to volume expansion in formerly preeclamptic women with low plasma volume. Reprod Sci. Jan 2009;16(1):105-112.

6. Rang $\mathrm{S}$, Wolf $\mathrm{H}$, van Montfrans GA, Karemaker JM. Serial assessment of cardiovascular control shows early signs of developing pre-eclampsia. J Hypertens. Feb 2004;22(2):369-376.

7. Courtar DA, Spaanderman ME, Aardenburg R, Janssen BJ, Peeters LL. Low plasma volume coincides with sympathetic hyperactivity and reduced baroreflex sensitivity in formerly preeclamptic patients. J Soc Gynecol Investig. Jan 2006;13(1):48-52.

8. Lang RM, Pridjian G, Feldman T, Neumann A, Lindheimer M, Borow KM. Left ventricular mechanics in preeclampsia. Am Heart J. Jun 1991;121(6 Pt 1):17681775.

9. Melchiorre K, Thilaganathan B. Maternal cardiac function in preeclampsia. Curr Opin Obstet Gynecol. Dec 2011;23(6):440-447.

10. Melchiorre K, Sutherland GR, Liberati M, Thilaganathan B. Preeclampsia is associated with persistent postpartum cardiovascular impairment. Hypertension. Oct 2011;58(4):709-715.

11. Report of the National High Blood Pressure Education Program Working Group on High Blood Pressure in Pregnancy. Am J Obstet Gynecol. Jul 2000;183(1):S1S22.

12. Visser GH, Eilers PH, Elferink-Stinkens PM, Merkus HM, Wit JM. New Dutch reference curves for birthweight by gestational age. Early Hum Dev. Dec 2009;85(12):737-744.

13. Deurenberg P, Weststrate JA, Seidell JC. Body mass index as a measure of body fatness: age- and sex-specific prediction formulas. Br J Nutr. Mar 1991;65(2):105114.

14. DuBois D, DuBois EF. A formula to estimate the approximate surface area if height and weight be known. Arch Int Med. 1916;17:863-871.

15. Aardenburg R, Spaanderman ME, Courtar DA, van Eijndhoven HW, de Leeuw PW, Peeters LL. A subnormal plasma volume in formerly preeclamptic women is associated with a low venous capacitance. J Soc Gynecol Investig. Feb 2005;12(2):107-111. 
16. Recommended methods for measurement of red-cell and plasma volume J Nucl Med. Aug 1980;21(8):793-800.

17. van Kreel BK, van Beek E, Spaanderman ME, Peeters LL. A new method for plasma volume measurements with unlabeled dextran-70 instead of $125 \mathrm{I}$-labeled albumin as an indicator. Clinica chimica acta; international journal of clinical chemistry. Jul 6 1998;275(1):71-80.

18. Aardenburg R, Spaanderman ME, Ekhart TH, van Eijndhoven HW, van der Heijden OW, Peeters LL. Low plasma volume following pregnancy complicated by preeclampsia predisposes for hypertensive disease in a next pregnancy. Br J Obstet Gynaecol. Nov 2003;110(11):1001-1006.

19. Aardenburg R, Spaanderman ME, van Eijndhoven HW, de Leeuw PW, Peeters LL. Formerly preeclamptic women with a subnormal plasma volume are unable to maintain a rise in stroke volume during moderate exercise. J Soc Gynecol Investig. Dec 2005;12(8):599-603.

20. Sahn DJ, DeMaria A, Kisslo J, Weyman A. Recommendations regarding quantitation in M-mode echocardiography: results of a survey of echocardiographic measurements. Circulation. Dec 1978;58(6):1072-1083.

21. Devereux RB, Casale PN, Kligfield P, et al. Performance of primary and derived Mmode echocardiographic measurements for detection of left ventricular hypertrophy in necropsied subjects and in patients with systemic hypertension, mitral regurgitation and dilated cardiomyopathy. Am J Cardiol. Jun 1 1986;57(15):1388-1393.

22. de Simone G, Daniels SR, Devereux RB, et al. Left ventricular mass and body size in normotensive children and adults: assessment of allometric relations and impact of overweight. J Am Coll Cardiol. Nov 1 1992;20(5):1251-1260.

23. Allison PD. Missing data techniques for structural equation modeling. J Abnorm Psychol. Nov 2003;112(4):545-557.

24. Palatini P. Heart rate and the cardiometabolic risk. Curr Hypertens Rep. Jun 2013;15(3):253-259.

25. Duvekot JJ, Cheriex EC, Pieters FA, Menheere PP, Peeters LH. Early pregnancy changes in hemodynamics and volume homeostasis are consecutive adjustments triggered by a primary fall in systemic vascular tone. Am J Obstet Gynecol. Dec 1993;169(6):1382-1392.

26. Simmons LA, Gillin AG, Jeremy RW. Structural and functional changes in left ventricle during normotensive and preeclamptic pregnancy. Am J Physiol Heart Circ Physiol. Oct 2002;283(4):H1627-1633.

27. Campos O. Doppler Echocardiography During Pregnancy: Physiological and Abnormal Findings. Echocardiography. Mar 1996;13(2):135-146.

28. Borghi C, Esposti DD, Immordino V, et al. Relationship of systemic hemodynamics, left ventricular structure and function, and plasma natriuretic peptide concentrations during pregnancy complicated by preeclampsia. Am J Obstet Gynecol. Jul 2000;183(1):140-147.

29. Miwa K, Fujita M. Cardiovascular dysfunction with low cardiac output due to a small heart in patients with chronic fatigue syndrome. Internal medicine. 2009;48(21):1849-1854. 
30. Simpson EH. The interpretation of interaction in Contingency Tables. Journal of the Royal Statistical Society. 1951;13(238-241).

31. American College of $\mathrm{O}$, Gynecologists, Task Force on Hypertension in $\mathrm{P}$. Hypertension in pregnancy. Report of the American College of Obstetricians and Gynecologists' Task Force on Hypertension in Pregnancy. Obstet Gynecol. Nov 2013;122(5):1122-1131. 


\section{Chapter 3:}

\section{Age-related alterations in cardiac geometry in}

formerly preeclamptic women and healthy parous controls; an explorative study

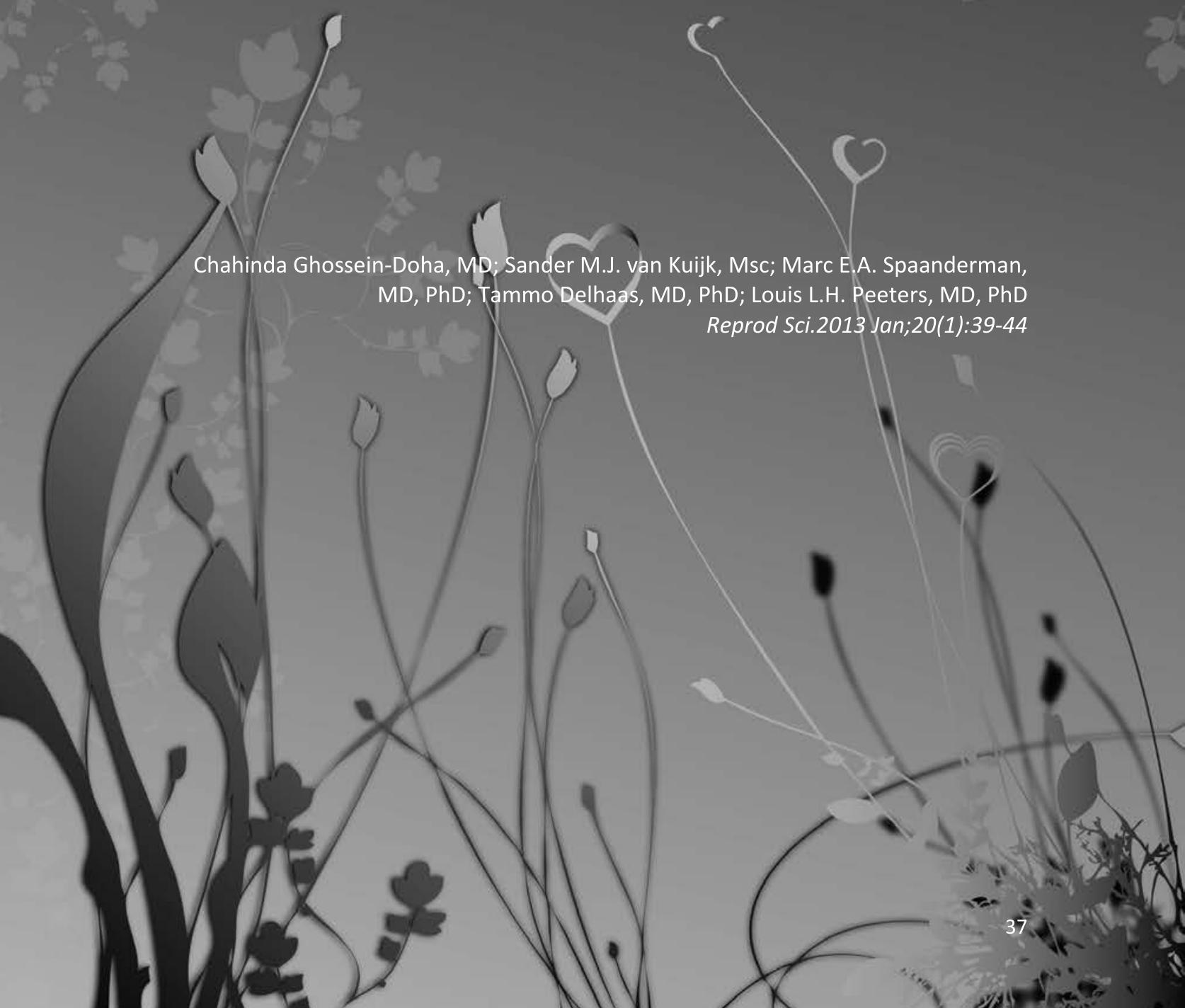




\section{Abstract}

Introduction: Preeclamptic pregnancies induce concentric left-ventricular hypertrophy instead of eccentric left-ventricular hypertrophy as seen in healthy pregnancies. Although these differences persist for at least several months postpartum, the longterm fate of these changes is unknown.

Objective: To explore the age-related changes in cardiovascular structure and function in formerly preeclamptic women relative to parous controls.

Methods: Twenty formerly preeclamptic women and 8 parous controls underwent two echocardiograms at 1 and 14 years postpartum. With the non-parametric MannWhitney $U$ test and the Wilcoxon Signed Ranks test, we analysed the in-betweengroups differences in cardiac structure and function at both time points as well as the time-related changes in these indices.

Results: Left-ventricular geometry and dimensions, and systolic function were comparable in the two study groups at both time points. The age related decline in E/A ratio and increase in intra-ventricular septum thickness were noted within both groups over time, without appreciable differences between groups.

Conclusion: A history of preeclampsia does not affect the age-dependent cardiac remodeling over a period of 14 years. 


\section{Introduction}

Preeclampsia (PE), a hypertensive pregnancy disorder complicating $2-5 \%$ of all pregnancies ${ }^{1}$, is characterized by new-onset hypertension accompanied by new-onset proteinuria. It is not only associated with perinatal complications, but also with longterm maternal cardiovascular morbidity. ${ }^{2}$ Nevertheless, the factors contributing to the higher prevalence of cardiovascular disorders (CVD) in formerly preeclamptic women are only partly identified. Moreover, there is accumulating evidence for hemodynamic and cardiovascular differences in the postpartum period after a preeclamptic and an uneventful pregnancy. ${ }^{3,4}$

In normal pregnancy left-ventricular (LV) mass and dimensions begin to increase in the first trimester. This so-called eccentric cardiac remodeling that resolves in the first weeks postpartum (pp) resembles the one in athletes. ${ }^{3,-7}$ In contrast, preeclamptic women also elicit an increase in LV mass during pregnancy, but with a less pronounced left-ventricular widening. This so called concentric cardiac remodeling seems to develop after the clinical onset of PE and persists for at least several months postpartum. ${ }^{3,8,9}$ Concentric cardiac remodeling is also known to develop in response to chronic hypertension. Since PE is diagnosed on the basis of new-onset hypertension, it is tempting to expect that concentric cardiac hypertrophy develops after its clinical onset. ${ }^{10}$ Concentric cardiac remodeling is known to be positively associated with a higher prevalence of CVD. Several studies have therefore explored the postpartum fate of this concentric left-ventricular remodeling in formerly preeclamptic women. However, the studied follow-up period lasted 12-16 months at most. ${ }^{4,8}$ This implies that none of the studies explored the age-related fate of the cardiac remodeling in formerly preeclamptic women.

This study was designed to explore the postpartum changes in cardiac geometry and dimensions, as well as in cardiac function over a prolonged period in formerly preeclamptic women relative to those in parous controls. We hypothesized that a history of PE is associated with altered cardiac aging relative to that in women with a history of only normal pregnancies.

\section{Methods}

\section{Study population}

The study protocol was approved by the Medical Ethical Committee of Maastricht University Medical Centre (MEC 08-2-130). In this longitudinal explorative study, we assessed cardiac geometry and dimensions as well as cardiac function in women with a history of PE and parous controls approximately at 1 and 14 years pp. PE was diagnosed according to the criteria of the International Society for the Study of Hypertension in Pregnancy. ${ }^{10}$ Early-onset PE is defined as PE diagnosed before 34 weeks pregnancy. We considered newborns to be small-for-gestational-age (SGA) if their birth weight was below the $5^{\text {th }}$ percentile according to the birth weight reference curves of the Perinatal Registry in the Netherlands. ${ }^{11}$ Hypertension was diagnosed on the basis of a mean systolic blood pressure $\geq 140 \mathrm{mmHg}$ and/or a mean diastolic arterial blood pressure $\geq 90 \mathrm{mmHg}$. 
Women who participated in a previous study on renal and cardiovascular functions in the period between 1996 and 1999 were invited to participate in this follow-up study. ${ }^{12}$ At that time ten controls were recruited by advertisement, whereas 39 women with a history of PE were recruited at the outpatient clinic at their postpartum checkup visit. Most of them experienced a severe form of PE, which could be either an earlyonset PE or PE complicated by fetal growth restriction or fetal demise. We invited all 10 controls and selected those 33 out of 39 women who had neither pre-existing renal disease nor (pre-pregnancy) hypertension. Three women had moved and could not be traced, one woman died of pulmonary embolism and one woman was currently undergoing chemotherapy. Furthermore, 8 formerly preeclamptic women and 2 controls declined participation, because of lack of time and/or emotional reasons. Eventually, 20 women with a history of PE and 8 parous controls agreed to participate in this follow-up study and gave informed consent before enrolment.

\section{Measurements}

At 1 and 14 years pp, we performed a similar set of measurements. Both measurement sessions took place in the mid-follicular phase of the menstrual cycle. Participants used a standardized sodium diet $\left(100 \mathrm{mmol}\right.$ sodium $\cdot$ day $\left.^{-1}\right)$ in the week preceding the measurement. Antihypertensive drugs ( $n=7$ in $P E$ group and $n=1$ in Control group) were discontinued 2 weeks earlier. During the 10 hours before the measurements the participants refrained from drinking caffeine- or alcohol-containing beverages, and from smoking and eating.

The measurement session started at 8:00 am in a temperature-controlled room $( \pm 24$ C). Throughout the measurements, participants were lying on a comfortable bed. After an acclimatization period of 30 minutes, we recorded arterial blood pressure for a period of 30 minutes at 3-minute intervals using a semi-automatic oscillometric device (Dinamap Vital Signs Monitor 1846; Critikon, Tampa, Florida, USA) in standardized environmental conditions with external disturbances kept to a minimum. We obtained the BMI by dividing body weight $(\mathrm{kg})$ by the squared height $\left(\mathrm{m}^{2}\right)$. An estimate for total fat mass was obtained by multiplying height $(\mathrm{m})$ with $(1.2 * \mathrm{BMI}+$ $0.23 *$ age -5.4 ). Lean Body Mass (LBM) was obtained by subtracting total fat mass from body weight. Body surface area (BSA) was calculated as follows: BSA $\left(\mathrm{m}^{2}\right)=$ 0.007184 . height $(\mathrm{cm})^{0.725}$. weight $(\mathrm{kg})^{0.425}$. Plasma volume $(\mathrm{PV}, \mathrm{mL} \cdot[\mathrm{kg}$ lean body mass] ${ }^{-1}$ ) was estimated using the indicator dilution technique using $1^{125}$-labelled albumin as indicator as detailed elsewhere. ${ }^{13}$

\section{Echocardiographic Evaluation}

After the PV measurement followed by 5 minutes rest, we assessed cardiac structure and function using cardiac ultrasound while the participant laid on her back in semi-left lateral position. We used a phased-array echocardiographic Doppler system (HewlettPackard Sonos 2000 and 2500; Hewlett-Packard Company, Palo Alto, California, USA) to perform the measurement at one year postpartum, and a Philips IE 33 system (Philips Medical System, Best, The Netherlands) to perform the measurement at 14 years postpartum. All data were stored on disk and the analysis was performed offline using specific software (Excelera, Philips, the Netherlands) by a single observer (CG). 
The echocardiography was performed according to the guidelines of the American Society of Echocardiography to measure relevant cardiac indices. ${ }^{14}$ Using M-mode in the parasternal long-axis view, we measured left-ventricular end-diastolic and endsystolic diameters (LVEDd, LVESd, mm), end-diastolic wall thickness of the interventricular septum (IVST, mm), and posterior-wall thickness (PWT). We estimated leftventricular mass (LVM, g) using the Devereux formula ${ }^{15}$ and indexed it for height in meters $^{2.7}$ as follows: LVMi $=\mathrm{LVM} \cdot \mathrm{m}^{-2.7} .^{16}$ The relative wall thickness (RWT) was calculated by the following formula: RWT = (IVST + PWT)/LVEDd. Fractional shortening (FS) was calculated by the formula: (LVEDd - LVESd) / LVEDd.

\section{Systolic function}

Left-ventricular systolic function was evaluated as follows: heart rate (HR, beats/min) was obtained by taking the reciprocal of the mean of between 3 and 5 consecutive RRintervals (sec) on the electrocardiogram and multiplied by 60 . The mean aortic Velocity Time Integral (VTI), measured by averaging the outer edge tracings of 3-5 consecutive continuous wave (CW) Doppler registrations of the flow across the aortic valve, was multiplied with the cross-sectional area measured at the level of the aortic annulus in the parasternal long-axis view to obtain stroke volume (SV). We obtained cardiac output $\left(\mathrm{CO}, \mathrm{L} \cdot \mathrm{min}^{-1}\right)$ by multiplying SV with HR. Cardiac index $(\mathrm{Cl})$ was calculated by the formula: $\mathrm{Cl}=\mathrm{CO} \cdot \mathrm{BSA}^{-1}$. SV was also indexed for BSA to obtain SV index (SVi).

\section{Diastolic function}

Diastolic function was assessed using pulsed-wave (PW) Doppler echocardiography to obtain transmitral flow determined from the apical 4-chamber view. The PW Doppler sample volume $(5 \mathrm{~mm})$ was carefully positioned at the tip of the mitral valve leaflets. The sweep rate was set at $50 \mathrm{~mm} \cdot \mathrm{s}^{-1}$. E/A ratio was defined as the ratio of peak mitral flow velocity in early diastole $(E)$ and that during atrial contraction $(A)$. Doppler-derived indices were averaged from 3-5 consecutive cardiac cycles.

\section{Total Vascular Resistance}

Total peripheral vascular resistance (TPVR in dynes $\cdot \mathrm{sec} \cdot \mathrm{cm}^{-5}$ ) was obtained by taking the ratio of mean arterial pressure (MAP, $\mathrm{mmHg}$ ) and $\mathrm{CO}$, multiplied by 80 . TPVR was indexed for BSA to obtain TPVR index (TPVRi).

\section{Statistical analyses}

We compared both groups by the non-parametric Mann-Whitney $U$ test. Time-related changes were analysed using the Wilcoxon Signed Ranks test. Categorical data (incidence of SGA, early-onset PE, fetal demise, outcome subsequent pregnancy) were analyzed using the Chi-square test. All analyses were performed using SPSS version 17.0. A p-value of less than 0.05 was considered statistically significant. Data are presented as median with interquartile range unless stated otherwise. 


\section{Results}

Table 1 lists the characteristics and pregnancy outcome in both study groups. The group of formerly preeclamptic patients differed from the controls by a lower gestational age at birth in the index pregnancy along with a higher incidence of SGA infants. Seven former patients and one parous control had developed hypertension in the course of the 14 years follow-up.

Table 1: Demography and obstetrical history of the control group and the former PE group. Data are presented as median with Interquartile range (IQR) unless stated otherwise.

\begin{tabular}{|c|c|c|c|}
\hline & & $\begin{array}{c}\text { Controls } \\
(n=8)\end{array}$ & $\begin{array}{c}\text { Former PE } \\
(n=20)\end{array}$ \\
\hline \multirow[t]{2}{*}{ Age (y) } & 1 year pp & $33(32 ; 34$ & $31(30 ; 32)$ \\
\hline & 14 years $p p$ & $45(44 ; 47)$ & $43(42 ; 45)$ \\
\hline \multirow[t]{2}{*}{ Smoking n(\%) } & 1 year pp & $1(13)$ & $2(10)$ \\
\hline & 14 year $\mathrm{pp}$ & $3(38)$ & $1(5)$ \\
\hline \multirow[t]{2}{*}{ Hypertension n(\%) } & 1 year pp & - & $3(15)$ \\
\hline & 14 year pp & $1(13)$ & $7(35)$ \\
\hline \multirow[t]{2}{*}{ Parity (median, range) } & 1 year pp & $2(1-2)$ & $1(1-2)$ \\
\hline & 14 year pp & $3(2-4)$ & $2(1-4)$ \\
\hline \multirow[t]{2}{*}{$\mathrm{BMI}\left(\mathrm{kg} \cdot \mathrm{m}^{-2}\right)$} & 1 year pp & $21(19 ; 25)$ & $22(20 ; 26)$ \\
\hline & 14 year pp & $23(20 ; 27)$ & $24(23 ; 30)$ \\
\hline \multicolumn{4}{|l|}{ Index pregnancy } \\
\hline \multicolumn{2}{|c|}{ Gestational age at birth (wk) } & $40(38 ; 42)$ & $32(27 ; 36)$ * \\
\hline \multicolumn{2}{|c|}{ Birth weight $(\mathrm{g})$} & $3360(2800 ; 3645)$ & $1600(850 ; 2630)^{\star}$ \\
\hline \multicolumn{2}{|c|}{ Incidence SGA births n(\%) } & - & $5(25 \%)$ \\
\hline \multicolumn{2}{|c|}{ Early preeclampsia n(\%) } & - & $13 / 20(65 \%)$ \\
\hline \multicolumn{2}{|l|}{ Fetal demise $n(\%)$} & - & $5(25 \%)$ \\
\hline \multicolumn{4}{|l|}{ Subsequent pregnancy } \\
\hline \multicolumn{2}{|l|}{ Number of subjects } & $7(88 \%)$ & $17(85 \%)$ \\
\hline \multicolumn{2}{|l|}{ Uncomplicated } & $7(100 \%)$ & $9(45 \%)$ \\
\hline \multicolumn{2}{|l|}{ Gestational hypertension } & - & $4(20 \%)$ \\
\hline \multicolumn{2}{|l|}{ Preeclampsia } & - & $4(20 \%)$ \\
\hline
\end{tabular}

BMI: Body mass index; SGA: small-for-dates (birth weight < p5\%).

* $p<0.05$ compared to Controls

\section{Intergroup comparison at 1 and 14 years postpartum}

Both groups were comparable with respect to systolic- and diastolic functions (table 2: $\mathrm{SV}, \mathrm{SVi}, \mathrm{HR}, \mathrm{CO}, \mathrm{Cl}, \mathrm{E} / \mathrm{A}$ ratio, TPVR and TPVRi) and LV geometry and dimensions (table $3)$, at both 1 year and 14 years pp. 


\section{Intragroup comparison}

In neither subgroup, LVM, LAD, PWT and RWT had changed consistently in the period elapsed between the two measurements (table 3). In both study groups IVST had increased by a similar fraction, whereas LVESd and LVEDd had increased significantly only in the former PE group. We were unable to discern a statistical difference in the change with time in the cardiac indices between both study groups.

Systolic- and diastolic BP and MAP had increased significantly only in the former PE subgroup. In neither subgroup, we found a change in SV, SVi, HR, and CO in the interval between both measurements. Meanwhile, in both subgroups $\mathrm{E} / \mathrm{A}$ ratio, $\mathrm{Cl}$ and FS had decreased and TPVR and TPVRi had increased by a comparable fraction in the study period.

Table 2: Hemodynamic indices at 1 and 14 years post partum (pp) with changeover time.

Data are listed as median with Interquartile range (IQR)

\begin{tabular}{|c|c|c|c|c|c|}
\hline & & \multicolumn{2}{|c|}{1 year pp } & \multicolumn{2}{|c|}{14 years $p p$} \\
\hline & & $\begin{array}{c}\text { Controls } \\
(n=8)\end{array}$ & $\begin{array}{l}\text { Former PE } \\
\quad(n=20)\end{array}$ & $\begin{array}{c}\text { Controls } \\
(n=8)\end{array}$ & $\begin{array}{l}\text { Former PE } \\
\quad(n=20)\end{array}$ \\
\hline Systolic BP & $\Delta$ & $117(108 ; 126)$ & $119(112 ; 127)$ & $\begin{array}{c}128(110 ; 146) \\
11(-1 ; 30)\end{array}$ & $\begin{array}{c}129(113 ; 144)^{\dagger} \\
10(-3 ; 17)\end{array}$ \\
\hline Diastolic BP & $\Delta$ & $75(69 ; 78)$ & $73(67 ; 84)$ & $\begin{array}{c}78(68 ; 146) \\
3(-3 ; 11)\end{array}$ & $\begin{array}{c}78(70 ; 92)^{\dagger} \\
5(-2 ; 11)\end{array}$ \\
\hline MAP & $\Delta$ & $91(79 ; 95)$ & $88(81 ; 84)$ & $\begin{array}{c}97(85-104) \\
10(3 ; 18)\end{array}$ & $\begin{array}{c}98(84 ; 111)^{\dagger} \\
10(1 ; 13)\end{array}$ \\
\hline SV & $\Delta$ & $69(62 ; 74)$ & $75(69 ; 79)$ & $\begin{array}{c}70(65 ; 83) \\
3(-2 ; 9)\end{array}$ & $\begin{array}{l}75(68 ; 85) \\
0(-8 ; 12)\end{array}$ \\
\hline SVi & $\Delta$ & $42(37 ; 45)$ & $42(40 ; 48)$ & $\begin{array}{c}41(38 ; 49) \\
1(-3 ; 5)\end{array}$ & $\begin{array}{l}42(39 ; 47) \\
-4(-6 ; 5)\end{array}$ \\
\hline HR & $\Delta$ & $69(58 ; 72)$ & $67(60 ; 70)$ & $\begin{array}{c}63(53 ; 74) \\
-1(-8 ; 3)\end{array}$ & $\begin{array}{c}64(57 ; 68) \\
-1(-6 ; 2)\end{array}$ \\
\hline $\mathrm{CO}$ & $\Delta$ & $4.7(4.4 ; 5.1)$ & $5.4(4.8 ; 6.9)$ & $\begin{array}{c}4.4(4.0 ; 5.0) \\
-0.2(-0.8 ; 0.2)\end{array}$ & $\begin{array}{c}4.7(4.3 ; 5.2) \\
-0.4(-1.3 ; 0.2)\end{array}$ \\
\hline $\mathrm{Cl}$ & $\Delta$ & $2.9(2.7 ; 3.2)$ & $3.2(2.8 ; 3.4)$ & $\begin{array}{c}2.6(2.3 ; 3.2) \\
-0.2(-0.6 ; 0.0)\end{array}$ & $\begin{array}{l}2.6(2.4 ; 3.0)^{\dagger} \\
-0.4(-0.8 ; 1.0)\end{array}$ \\
\hline E/A ratio & $\Delta$ & $1.7(1.5 ; 2.0)$ & $1.5(1.3 ; 1.8)$ & $\begin{array}{c}1.2(1.1 ; 1.3)^{\dagger} \\
-0.3(-0.6 ;-0.2)\end{array}$ & $\begin{array}{l}1.3(1.0 ; 1.4)^{\dagger} \\
-0.3(-0.7 ; 0.0)\end{array}$ \\
\hline TPVR & $\Delta$ & $1406(1203 ; 1616)$ & $1329(1104 ; 1422)$ & $\begin{array}{c}1733(1483 ; 1973)^{\dagger} \\
355(79 ; 523)\end{array}$ & $\begin{array}{c}1584(1350 ; 1753)^{\dagger} \\
241(-34 ; 623)\end{array}$ \\
\hline TPVRi & $\Delta$ & $843(719 ; 979)$ & $746(627 ; 852)$ & $\begin{array}{c}1031(912 ; 1136)^{\dagger} \\
211(30 ; 258)\end{array}$ & $\begin{array}{c}890(772 ; 967)^{\dagger} \\
71(-38 ; 325)\end{array}$ \\
\hline
\end{tabular}

BP: Blood pressure (mmHg); MAP: Mean Arterial Pressure (mmHg); SV: Stroke Volume $(\mathrm{mL}) ;$ SVi: SV index (mL-m-2); HR: Heart Rate (beats ·min-1); CO: Cardiac Output (L.min-1); Cl: Cardiac Index (L.min-1.m-2); EA ratio: early transmitral diastolic flow velocity/flow velocity during atrial contraction ratio; TPVR: Total peripheral vascular resistance $($ dyn. $\mathrm{S} \cdot \mathrm{cm}-5)$; TPVRi: TPVR index ((dyn.s.cm-5.m-2); $\Delta$ : median change over time

* $p<0.05$ compared to Controls $\dagger p<0.05$ compared to 1 year $p p$ 
Table 3: Left ventricular geometry and dimensions at 1 and 14 years post partum (pp) with mean change over time. Data are listed as median with interquartile range (IQR).

\begin{tabular}{|c|c|c|c|c|c|}
\hline & \multicolumn{2}{|c|}{1 year $p p$} & \multicolumn{2}{|c|}{14 years pp } \\
\hline & & $\begin{array}{c}\text { Controls } \\
(n=8)\end{array}$ & $\begin{array}{l}\text { Former PE } \\
(n=20)\end{array}$ & $\begin{array}{c}\text { Controls } \\
(n=8)\end{array}$ & $\begin{array}{l}\text { Former PE } \\
(n=20)\end{array}$ \\
\hline LAD & $\Delta$ & $34(32 ; 36)$ & $36(32 ; 37)$ & $\begin{array}{c}35(31 ; 41) \\
1(-3 ; 4)\end{array}$ & $\begin{array}{c}36(33 ; 39) \\
0(-2 ; 2)\end{array}$ \\
\hline FS & $\Delta$ & $36(34 ; 38)$ & $34(33 ; 37)$ & $\begin{array}{c}32(30 ; 36)^{\dagger} \\
-3(-5 ;-1)\end{array}$ & $\begin{array}{c}33(31 ; 36)^{\dagger} \\
-2(-4 ;-1)\end{array}$ \\
\hline IVST & $\Delta$ & $7.0(7.0 ; 7.8)$ & $7.0(7.0 ; 8.0)$ & $\begin{array}{l}8.0(7.6 ; 8.8)^{\dagger} \\
1.0(0.6 ; 1.2)\end{array}$ & $\begin{array}{l}7.7(7.2 ; 8.1)^{\dagger} \\
0.4(0.0 ; 1.0)\end{array}$ \\
\hline PWT & $\Delta$ & $8.0(7.0 ; 8.0)$ & $8.1(7.0 ; 8.2)$ & $\begin{array}{c}7.7(7.0 ; 7.9) \\
-0.2(-0.9 ; 0.3)\end{array}$ & $\begin{array}{c}7.9(7.0 ; 8.2) \\
-0.1(-0.8 ; 0.8)\end{array}$ \\
\hline LVM & $\Delta$ & $122(114 ; 144)$ & $134(116 ; 146)$ & $\begin{array}{c}110(105 ; 139) \\
-13(-16 ;-1)\end{array}$ & $\begin{array}{c}126(102 ; 150) \\
-10(-22 ; 11)\end{array}$ \\
\hline LVMi & $\Delta$ & $31(29 ; 38)$ & $32(28 ; 38)$ & $\begin{array}{c}29(26 ; 35) \\
-3(-4 ;-1)\end{array}$ & $\begin{array}{c}29(24 ; 40) \\
-3(-5 ; 4)\end{array}$ \\
\hline RWT & $\Delta$ & $0.32(30 ; 37)$ & $0.33(0.32 ; 0.35)$ & $\begin{array}{c}0.35(0.32 ; 0.36) \\
0.01(-0.04 ; 0.04)\end{array}$ & $\begin{array}{l}0.32(0.30 ; 0.34) \\
0.01(-0.03 ; 0.02)\end{array}$ \\
\hline LVEDd & $\Delta$ & $45(42 ; 47)$ & $46(43 ; 48)$ & $\begin{array}{c}44(42 ; 49) \\
1(-2 ; 3)\end{array}$ & $\begin{array}{c}47(45 ; 50)^{\dagger} \\
2(-1 ; 4)\end{array}$ \\
\hline LVESd & $\Delta$ & $30(27 ; 30)$ & $30(27 ; 32)$ & $\begin{array}{c}30(28 ; 33) \\
2(0 ; 3)\end{array}$ & $\begin{array}{c}32\left(30 ; 33^{\dagger}\right. \\
1.5(0.0 ; 3.8)\end{array}$ \\
\hline
\end{tabular}

LAD: left atrial diameter (cm); FS: fractional shortening (\%); IVST: Inter-Ventricular Septum Thickness (mm); PWT: Left Ventricular Posterior Wall Thickness (mm); LVM: Left Ventricular Mass (g); LVMi: Left ventricular mass index $\left(\mathrm{g} \cdot \mathrm{m}^{-2.7}\right)$; RWT: Relative Wall Thickness ( ); LVEDd: Left Ventricular End Diastolic Diameter (mm); LVESd: Left Ventricular EndSystolic Diameter (mm); $\Delta$ : median change over time.

* $p<0.05$ compared to Controls ${ }^{\dagger} p<0.05$ compared to 1 year $p p$. 


\section{Discussion}

To the best of our knowledge, this is the first longitudinal study evaluating the postpreeclampsia cardiac changes over such a long follow-up period. Despite the small study population, this study provides novel information on the fate of the cardiac remodeling and function in formerly preeclamptic women and parous controls after a follow-up period of 14 years. Most importantly, this study showed that the changes in cardiac geometry, dimensions and function were similar in both groups. Moreover, both groups showed a similar decline in FS and E/A ratio. As all women were still premenopausal, the effect of menopause on the cardiovascular system did not act as a potential confounder.

Large retrospective epidemiological studies have consistently demonstrated an elevated risk for many types of CVD in women with a history of PE. ${ }^{2}$ The mechanisms responsible for a higher risk of CVD in women with a history of PE are only partly understood. Our study provides some indirect evidence that differences in cardiac geometry, dimensions and/or function do not measurably contribute to the elevated risk of CVD in former PE patients.

\section{Differences between both groups at 1 and 14 years $p p$}

At one year postpartum we were unable to discern a measurable difference in LV geometry between former PE patients and controls. Postpartum studies up to 6 months pp, found a thicker PWT and IVST and increased LVMi and RWT in the former PE group., ${ }^{3,17}$ We found no differences in LV geometry at 1 year pp which is opposed to a previous study that found an increased RWT and more hypertrophied ventricles and a lower EA ratio in formerly preeclamptics at one year pp compared to controls. ${ }^{8}$ However, this difference in findings could be due to differences in inclusion criteria or due to our small sample size.

We were also unable to discern a consistent difference in systolic function between both groups at 1 year pp, which is in line with previous findings. ${ }^{4}$ As FS and LVEDd did not differ between both study groups, it is likely that both study groups have preserved systolic cardiac functioning. Women who experienced preterm preeclampsia showed a higher incidence of asymptomatic left ventricular moderate-severe dysfunction/hypertrophy at one year postpartum, known as stage B heart failure compared to matched controls. ${ }^{18}$ This differs from what was observed in women with a history of late-onset or term preeclampsia, who did not show a higher incidence of stage B heart failure at 1 year postpartum compared to matched controls. The difference in the incidence of stage $B$ heart failure between women with early-onset and late-onset preeclampsia suggests a different postpartum remodeling. It has also been reported that normotensive women who experienced a preeclamptic pregnancy 13-18 years earlier have an increased myocardial performance index (MPI) and decreased ejection time. ${ }^{19}$ However, SV and CO as single parameters of systolic function do not differ between formerly preeclamptic women and healthy controls $\mathrm{s}^{4,19}$ which is in line with our findings.

In our study, 7 women in the PE group and 1 in the control group were diagnosed with chronic hypertension and were using antihypertensive medication. We asked these patients to discontinue their medication two weeks prior to the echocardiographic 
measurements. Although timely discontinuation of these drugs prior to measurement excludes the short-term effects of antihypertensive medication on cardiac responses, it cannot be ruled out that the preceding chronic use of this medication may have influenced the structure of the heart. If so, the antihypertensive effect of the medication can be expected to have diminished the hypertension-related effects on the cardiac structure. Not only hypertension and antihypertensive medication, but also metabolic syndrome plays an important role in the postpartum changes in cardiac structure and function. It has been stated that the metabolic syndrome persisting after preeclampsia is likely to raise the incidence of diastolic dysfunction in formerly preeclamptic women. ${ }^{20}$ However, information on the EA ratio without concomitant support of other validated diagnostic algorithms to assess diastolic function, is insufficient to draw firm conclusions on diastolic function.

\section{Age-dependent changes}

Our findings support the concept that in women the increase in IVST with time is larger than that of the posterior wall, irrespective of whether or not they experienced a PE pregnancy. ${ }^{21}$ The parous controls did neither elicit time-dependent changes in LV dimensions and volumes, nor in $\mathrm{CO}, \mathrm{HR}, \mathrm{SV}$ and $\mathrm{Cl}$, an observation in line with previous reports. $^{22,23,24}$ In fact, in healthy adults neither LV systolic and diastolic dimensions, ${ }^{22,}$ ${ }^{23}$ nor CO and SV ${ }^{24}$ change with aging.

Our observation of an age-related decline in E/A ratio confirms previous findings. ${ }^{25} \mathrm{We}$ noticed that the rate of decline in E/A ratio did not differ between formerly PE women and parous controls. Apparently, aging is accompanied by loss in cardiac compliance, but at comparable rate in formerly preeclamptic women and healthy parous controls. Our primary objective in this study was to explore whether a history of PE as a single criterion affects the response of the cardiac geometry and function to aging in formerly PE patients without taking the severity of PE into consideration. Early-onset preeclampsia and recurrent preeclampsia are known to raise the risk for CVD in former PE patients relative to controls. ${ }^{2}$ Of the women in our PE group, $65 \%$ had a history of early-onset PE and 20\% experienced recurrent PE. However, despite the fact that more than half of our population consisted of a high risk population, we did not find significant differences between the PE and control groups. The lack of significant differences between the two groups makes it even less likely to find significant differences in cases of mild (late-onset) PE or in the absence of pre-existing conditions. Whether we would find persistent remodeling if we studied only a high risk subpopulation requires further study.

This longitudinal prospective study with paired measurements covers a period of as long as 14 years. Because of the longitudinal design with a very long follow-up period, we were unable to include a large sample of women. The modest sample size of this study mainly weakens the intergroup analysis. However, the use of a repeated measures design, which makes very efficient use of the data, increases the sensitivity of the intragroup results. Despite the statistically efficient design, our results should be interpreted with caution and require confirmation in a larger study.

In conclusion, this study provides evidence for no appreciable long-term negative effects of PE as a single criterion on cardiac structure and function. To unravel the 
exact mechanism contributing to the higher incidence of hypertension and other CVD after PE, an adequately powered long-term follow-up study is needed preferably in a population of primarily women with the highest risk of CVD, more specifically those who experienced early-onset PE.

\section{Acknowledgements}

We would like to thank I. Schreij and T. Ekhart and J. Spaan, MD, for their contribution in the data collection. 


\section{References}

1. Sibai B, Dekker G, Kupferminc M. Pre-eclampsia. Lancet. Feb 26-Mar 4 2005;365(9461):785-799.

2. Bellamy L, Casas JP, Hingorani AD, Williams DJ. Pre-eclampsia and risk of cardiovascular disease and cancer in later life: systematic review and metaanalysis. Bmj. Nov 10 2007;335(7627):974.

3. Simmons LA, Gillin AG, Jeremy RW. Structural and functional changes in left ventricle during normotensive and preeclamptic pregnancy. Am J Physiol Heart Circ Physiol. Oct 2002;283(4):H1627-1633.

4. Evans CS, Gooch L, Flotta D, et al. Cardiovascular system during the postpartum state in women with a history of preeclampsia. Hypertension. Jul;58(1):57-62.

5. Campos O. Doppler Echocardiography During Pregnancy: Physiological and Abnormal Findings. Echocardiography. Mar 1996;13(2):135-146.

6. Robson SC, Hunter S, Boys RJ, Dunlop W. Serial study of factors influencing changes in cardiac output during human pregnancy. Am J Physiol. Apr 1989;256(4 Pt 2):H1060-1065.

7. Lang RM, Pridjian G, Feldman T, Neumann A, Lindheimer M, Borow KM. Left ventricular mechanics in preeclampsia. Am Heart J. Jun 1991;121(6 Pt 1):17681775.

8. Valensise H, Vasapollo B, Gagliardi G, Novelli GP. Early and late preeclampsia: two different maternal hemodynamic states in the latent phase of the disease. Hypertension. Nov 2008;52(5):873-880.

9. Vasapollo B, Novelli GP, Valensise H. Total vascular resistance and left ventricular morphology as screening tools for complications in pregnancy. Hypertension. Apr 2008;51(4):1020-1026.

10. Perry IJ, Beevers DG. The definition of pre-eclampsia. Br J Obstet Gynaecol. Jul 1994;101(7):587-591.

11. Visser GH, Eilers PH, Elferink-Stinkens PM, Merkus HM, Wit JM. New Dutch reference curves for birthweight by gestational age. Early Hum Dev. Dec 2009;85(12):737-744.

12. Spaanderman ME, Van Beek E, Ekhart TH, et al. Changes in hemodynamic parameters and volume homeostasis with the menstrual cycle among women with a history of preeclampsia. Am J Obstet Gynecol. May 2000;182(5):11271134.

13. Recommended methods for measurement of red-cell and plasma volume: International Committee for Standardization in Haematology. J Nucl Med. Aug 1980;21(8):793-800.

14. Sahn DJ, DeMaria A, Kisslo J, Weyman A. Recommendations regarding quantitation in M-mode echocardiography: results of a survey of echocardiographic measurements. Circulation. Dec 1978;58(6):1072-1083.

15. Devereux RB, Casale PN, Kligfield P, et al. Performance of primary and derived Mmode echocardiographic measurements for detection of left ventricular hypertrophy in necropsied subjects and in patients with systemic hypertension, mitral regurgitation and dilated cardiomyopathy. Am J Cardiol. Jun 1 1986;57(15):1388-1393. 
16. de Simone G, Daniels SR, Devereux RB, et al. Left ventricular mass and body size in normotensive children and adults: assessment of allometric relations and impact of overweight. J Am Coll Cardiol. Nov 1 1992;20(5):1251-1260.

17. Rafik Hamad R, Larsson A, Pernow J, Bremme K, Eriksson MJ. Assessment of left ventricular structure and function in preeclampsia by echocardiography and cardiovascular biomarkers. J Hypertens. Nov 2009;27(11):2257-2264.

18. Melchiorre K, Sutherland GR, Liberati M, Thilaganathan B. Preeclampsia is associated with persistent postpartum cardiovascular impairment. Hypertension. Oct 2011;58(4):709-715.

19. Strobl I, Windbichler $G$, Strasak $A$, et al. Left ventricular function many years after recovery from pre-eclampsia. BJOG. Jan 2011;118(1):76-83.

20. Zandstra $M$, Stekkinger $E$, van der Vlugt MJ, van Dijk AP, Lotgering FK, Spaanderman ME. Cardiac diastolic dysfunction and metabolic syndrome in young women after placental syndrome. Obstet Gynecol. Jan 2010;115(1):101108.

21. Kovacs G, Berghold A, Scheidl S, Olschewski H. Pulmonary arterial pressure during rest and exercise in healthy subjects: a systematic review. Eur Respir J. Oct 2009;34(4):888-894.

22. Gerstenblith G, Frederiksen J, Yin FC, Fortuin NJ, Lakatta EG, Weisfeldt ML. Echocardiographic assessment of a normal adult aging population. Circulation. Aug 1977;56(2):273-278.

23. Hees PS, Fleg JL, Lakatta EG, Shapiro EP. Left ventricular remodeling with age in normal men versus women: novel insights using three-dimensional magnetic resonance imaging. Am J Cardiol. Dec 1 2002;90(11):1231-1236.

24. Fleg JL, Shapiro EP, O'Connor F, Taube J, Goldberg AP, Lakatta EG. Left ventricular diastolic filling performance in older male athletes. Jama. May 3 1995;273(17):1371-1375. 

Part 2

Risk factors for hypertension and heart failure stage-B after preeclampsia 



\section{Chapter 4:}

\section{Hypertension after preeclampsia is preceded by changes in cardiac structure and function}

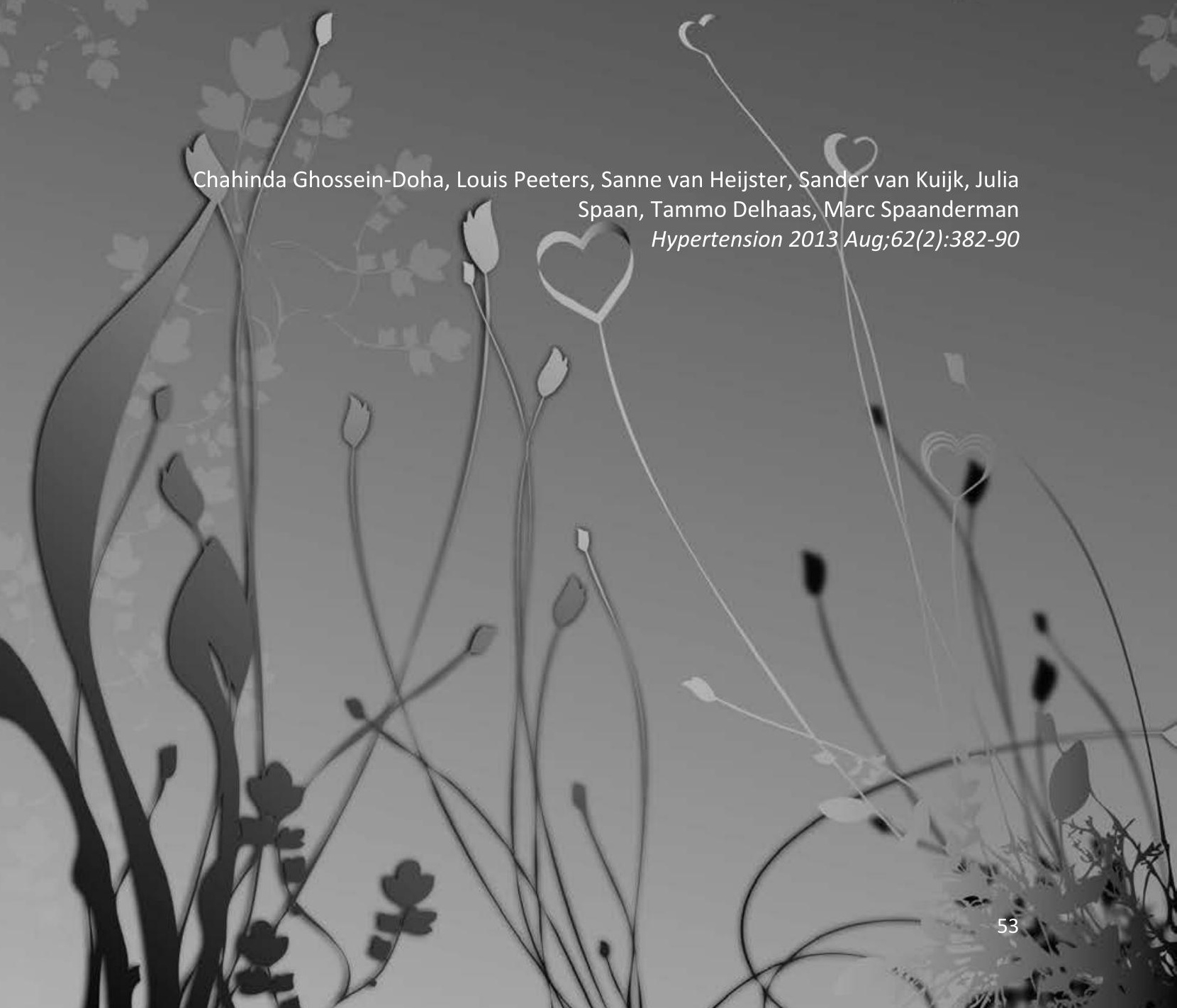




\begin{abstract}
Preeclampsia is associated with a 4-fold higher risk for developing remote chronic hypertension. Preeclampsia is accompanied by left ventricular hypertrophy and decreased diastolic function, which may or may not resolve postpartum. We tested the hypothesis that increased measures of cardiac geometry and decreased cardiac function persisting for at least 6 months postpartum in normotensive women with a history of preeclampsia precede the development of later chronic hypertension. Formerly preeclamptic women $(n=652)$ underwent echocardiography at 9 months (range: 6-19) postpartum. We excluded women with pre-existing hypertension ( $n=42)$, hypertension at the postpartum screening $(n=133)$ and those that did not return any checklist $(n=128)$. Eventually, 349 women were included. Remote health, was evaluated by a biennially checklist. We used Cox regression for analysis. Twenty-seven (8\%) normotensive women had developed chronic hypertension during a medium follow-up period of 6 years. At screening they differed from their counterparts who remained normotensive by Hazard Ratio for left ventricular mass index $(1.11 ; 95 \% \mathrm{Cl}$ $1.03-1.18)$, diastolic blood pressure $(1.13 ; 95 \% \mathrm{Cl} 1.06-1.20)$, systolic blood pressure (1.07; 95\% Cl 1.02-1.11), mean arterial pressure $(1.11 ; 95 \% \mathrm{Cl} 1.05-1.18)$, heart rate (1.05; 95\% Cl 1.01-1.10), and E/A ratio $(0.22 ; 95 \% \mathrm{Cl} 0.06-0.85)$. Backward stepwise analysis showed independent Hazard Ratio for left ventricular mass index and diastolic blood pressure: 1.08 (95\% Cl 1.01-1.16) and 1.13 (95\% Cl 1.06-1.21), respectively. In conclusion, the development of later chronic hypertension in initially normotensive formerly preeclamptic women is preceded by increased left ventricular mass index and diastolic blood pressure at postpartum screening.
\end{abstract}




\section{Introduction}

Preeclampsia (PE), a serious hypertensive pregnancy disorder complicating 2-5\% of all pregnancies, is characterized by new-onset hypertension along with de-novo proteinuria after 20 weeks of gestation ${ }^{1}$ or new onset proteinuria that develops after 20 weeks in women with preexisting hypertension. Women with a history of PE have an increased risk to develop chronic hypertension and related cardiovascular morbidity and mortality. ${ }^{2}$

$\mathrm{PE}$ is accompanied by concentric left ventricular remodeling along with diastolic dysfunction and reduced cardiac contractility. ${ }^{3}$ Accumulating evidence exists that women who experienced PE differ from their counterparts with uneventful pregnancies by persisting abnormal left ventricular geometry and decreased diastolic function. ${ }^{4,5}$ It may be that former patients with underlying susceptibility for CVD react to borderline blood pressure values by excessive cardiac remodeling that does not revert to normal after delivery. In this line of reasoning, the slight differences in left ventricular geometry and cardiac diastolic function may be an indication for an increased risk for chronic hypertension as an intermediate condition prior to later CVD. Therefore we tested the hypothesis that increased measures of cardiac geometry and decreased cardiac function persisting for at least 6 months postpartum in normotensive women with a history of PE precede the development of later chronic hypertension.

\section{Methods}

\section{Study population}

The Maastricht University Medical Centre (MUMC) Medical Ethics Committee approved our study protocol before patient enrolment (MEC 0-4-049). Since 1996 we perform postpartum screening at least 4 months postpartum in women who experienced a pregnancy complicated by a hypertensive disorder. The procedures followed were in accordance with institutional guidelines. Informed consent related to the use of clinically acquired data for scientific analysis is standard given at MUMC. Procedures adhere to the principles of the Declaration of Helsinki and Title 45, U.S. Code of Federal Regulations, Part 46, Protection of Human Subjects, Revised November 13, 2001, effective December 13, 2001. For the present study, we included all Caucasian primiparous women who were screened postpartum until 2008. PE was diagnosed on the basis of the criteria of the International Society for the Study of Hypertension in Pregnancy. ${ }^{7}$ Early-onset PE was defined as PE diagnosed prior to the $34^{\text {th }}$ week of pregnancy. Subjects were scheduled for screening at least 4 weeks after stopping breastfeeding. Patients were asked to discontinue the use of oral contraceptives. After the postpartum screening, all women received biennially questionnaires designed to evaluate their health status and possible subsequent pregnancies. We excluded women with pre-existing hypertension indicated by hypertension already present before 20 weeks pregnancy or hypertension still present at the time of postpartum screening $(n=42)$. Hypertension is diagnosed as systolic blood pressure (SysBP) $\geq 140 \mathrm{mmHg}$ and/or diastolic blood pressure (DiasBP) $\geq 90$ 
$\mathrm{mmHg}$, or when taking antihypertensive drugs. Women who did not complete or return any questionnaire were considered lost to follow-up. We took self-reported use of antihypertensive medication to define hypertension at follow-up.

\section{Measurements}

Ten hours before the measurement session, participants were asked to refrain from eating, drinking caffeine- or alcohol-containing beverages, and smoking. The measurement session started at 8:00 am in a temperature-controlled room ( $\pm 24 \stackrel{\circ}{\circ}$ ) with external disturbances kept to a minimum. Throughout the measurements, participants were lying on their back on a comfortable bed in a half-sitting position with their upperarm at heart height while the whole arm was in a relaxed position. After an acclimatization period of 30 minutes, we recorded arterial blood pressure at 3-minutes intervals for a period of 30 minutes using a semi-automatic oscillometric device (Dinamap Vital Signs Monitor 1846; Critikon, Tampa, Florida, USA) with a cuff size of $13.5 \times 30.7 \mathrm{~cm}$ if arm circumference ranged between $27.5-36.5 \mathrm{~cm}$ or a cuff size of $17 X 38.6 \mathrm{~cm}$ if arm circumference ranged between $35.5-46 \mathrm{~cm}$. BMI was obtained by dividing body weight $(\mathrm{kg})$ by the squared height $\left(\mathrm{m}^{2}\right)$. We obtained an estimate for total fat mass by multiplying height $(\mathrm{m})$ with [(1.2*BMI)+(0.23*age)-5.4]. Lean Body Mass (LBM, kg) was estimated by subtracting total fat mass from body weight. Body surface area (BSA) was calculated as follows:

BSA $\left(\mathrm{m}^{2}\right)=0.007184 \cdot$ height $(\mathrm{cm})^{0.725} \cdot$ weight $(\mathrm{kg})^{0.425}$.

\section{Echocardiographic measurements}

We assessed cardiac structure and function using a phased-array echocardiographic Doppler system (Hewlett-Packard Sonos 2000 and 2500; Hewlett-Packard Company, Palo Alto, Ca), and performed the two-dimensional, M-mode and Doppler echocardiography according to the guidelines of the American Society of Echocardiography. ${ }^{9}$ All data were analyzed offline using specific software (Excelera, Philips, The Netherlands). Using $\mathrm{M}$-mode in the parasternal long-axis view, we measured left ventricular end-diastolic and end-systolic diameters (LVEDd, LVESd, $\mathrm{mm}$ ), as well as end-diastolic thickness of the interventricular septum (IVST, $\mathrm{mm}$ ) and of the posterior wall (PWT, mm). We used the Devereux-formula ${ }^{10}$ to estimate left-ventricular mass (LVM, g) and indexed for height in meters ${ }^{2.7} .{ }^{11}$ Finally, we calculated relative wall thickness (RWT=(IVST+PWT)/LVEDd) and fractional shortening FS=(LVEDdLVESd)/LVEDd.

\section{Systolic function}

Left-ventricular systolic function was evaluated as follows: heart rate (HR, beats $\cdot \mathrm{min}^{-1}$ ) was obtained by taking the reciprocal of the mean of 3 to 5 consecutive RR-intervals on the electrocardiogram (sec) multiplied by 60 . The mean aortic Velocity Time Integral (VTI) was obtained from the average of the outer edge tracings of 3-5 consecutive continuous wave (CW) Doppler registrations of the aortic flow across the aortic valve. Stroke volume (SV, $\mathrm{ml}$ ) was derived by: VTI ' cross-sectional area of the aortic annulus in the parasternal long-axis view. Cardiac output $\left(\mathrm{CO}, \mathrm{L} \cdot \mathrm{min}^{-1}\right)$ was calculated as: SV'HR. 
Cardiac index $(\mathrm{Cl})$ was calculated by: $\mathrm{CO} \cdot \mathrm{BSA}^{-1}$. Left ventricular end-diastolic volume (EDV, $\mathrm{ml}$ ) and end-systolic volumes (ESV, $\mathrm{ml}$ ) were estimated using the Teichholzformula. ${ }^{12}$ Ejection fraction was calculated by $E F(\%)=((E D V-E S V) /(E D V)) \cdot 100 \%$.

\section{Diastolic function}

Diastolic function was assessed from the transmitral flow pattern obtained by pulsedwave (PW) Doppler echocardiography in the apical 4-chamber view. The PW Doppler sample volume $(5 \mathrm{~mm})$ was carefully positioned at the tip of the mitral valve leaflets. The sweep rate was set at $50 \mathrm{~mm} \cdot \mathrm{s}^{-1}$. E/A ratio was defined as the ratio of peak mitral flow velocity in early diastole (E) and that during atrial contraction (A). Doppler-derived indices were averaged over 3-5 consecutive cardiac cycles.

\section{Total Peripheral Vascular Resistance}

Total peripheral vascular resistance (TPVR in dynes $\left.\cdot \mathrm{sec} \cdot \mathrm{cm}^{-5}\right)$ was obtained by $(80$ mean arterial pressure (MAP, $\mathrm{mmHg})$ )/CO. TPVR was indexed for BSA to obtain TPVRi.

\section{Renal function}

Glomerular filtration rate (eGFR; $\mathrm{ml} / \mathrm{min}$ per $1.73 \mathrm{~m}^{2}$ ) was estimated using the Modification of Diet in Renal Disease formula: 32788 serum creatinine $(\mathrm{mol} / \mathrm{l})^{1.154}$. age (years) ${ }^{0.203} \cdot 0.742$. The presence of micro-albuminuria was defined as a urinary albumin excretion rate $\geq 3.5 \mathrm{~g} \mathrm{~mol}^{-1}$ creatinine.

\section{Statistical analysis}

We used Cox regression analysis to explore the associations between cardiac- and obstetric variables and the cumulative incidence of hypertension over a period of 12 years. We defined an event as the occurrence of hypertension during follow-up (yes/no). Time-to-event was defined as the time elapsed between the date of postpartum screening and the date of starting the use of antihypertensive medication or, if not available, by a date halfway the most recent follow-up interval. Patients who had not developed hypertension at the end of the study period were censored at the end of their follow-up time. Since the amount of missing values in the variables considered for analysis never exceeded $5 \%$, we did not use methods to impute these missing observations. Variables that showed significant associations $(p<0.05)$ in the univariate analysis were included in a multivariate Cox regression model. For the multivariate analysis, we used stepwise backward-elimination, based on the Wald test, using a p-value of 0.05 for elimination of variables. To deal with co-linearity we used only one variable if two variables measured the same parameter. Variables that remained in the Cox regression model after backward stepwise-elimination were used to create subgroups for different Kaplan Meier analyses. Subjects were stratified according to the presence of the highest-risk-quartile values versus all other patients. Due to a small percentage $(<5 \%)$ of missing values in our measurements, numbers at 2 , $4,6,8,10$ and 12 years could differ between those with BP and those with cardiac ultrasound measurements. Next, we used the log-rank test to test if the Kaplan Meier 
curves were significantly different. All computations were performed using SPSS version 20.0.

\section{Results}

Between 1996 and 2008, 652 primiparous women with a history of PE were evaluated. Of the 652 formerly preeclamptic women, 42 (6\%) had pre-existing hypertension, whereas at the postpartum screening, 133 (20\%) were hypertensive and 477 (73\%) normotensive (fig. 1). We sent a questionnaire to all normotensive women at least 18 months after the screening and this was repeated every two years. A total of 128 women did not return any completed questionnaire, but 349 women responded to at least one questionnaire. Of those responders, 322 (92\%) remained normotensive, while $27(8 \%)$ had developed chronic hypertension during the follow up. The median follow-up time was 6 (IQR 3-9) years. Also the 133 women who were hypertensive at postpartum screening received a questionnaire, and 74 responded to at least one questionnaire. Out of those 74 women who were hypertensive at postpartum screening, 14 did not report the use of antihypertensive medication at follow-up. The total prevalence of chronic hypertension after PE in our population of formerly preeclamptics that responded to a questionnaire is $87 / 432$ (20\%).

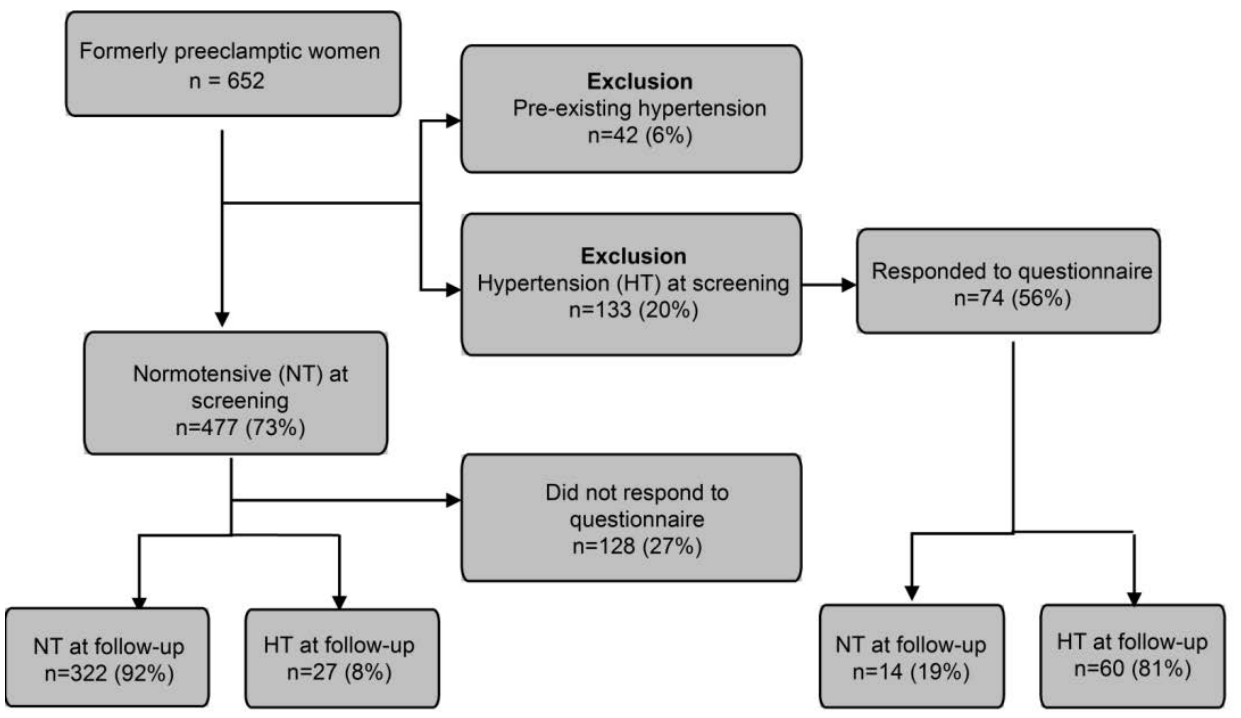

Figure 1: Flowchart of the study population

Table 1 shows the baseline characteristics of the women who developed hypertension at follow up and those who remained normotensive. All women were normotensive at postpartum screening. Women who developed chronic hypertension differed from those who remained normotensive by delivering their child with a lower birth weight at a shorter gestational age. 
Table 1: Mean values $( \pm$ SD) of cardiac indices in women with a history of preeclampsia that were normotensive at the time of postpartum screening and did or did not develop chronic hypertension. Groups were compared with the independent t-test. Binary variables were tested with the $\mathrm{X}^{2}$.

\begin{tabular}{|c|c|c|c|}
\hline Indices & $\begin{array}{l}\text { Normotensive group } \\
\qquad(\mathrm{n}=322)\end{array}$ & $\begin{array}{l}\text { Hypertensive group } \\
\qquad(\mathrm{n}=27)\end{array}$ & $\mathrm{p}$-value \\
\hline Age at PP screening (years) & $31 \pm 4$ & $31 \pm 3$ & 0.45 \\
\hline Months PP (median, IQR) & $10(6-20)$ & $10(8-19)$ & 0.85 \\
\hline Body mass index $\left(\mathrm{kg} / \mathrm{m}^{2}\right)$ & $25 \pm 4$ & $28 \pm 7$ & 0.06 \\
\hline Body surface area $\left(\mathrm{m}^{2}\right)$ & $1.8 \pm 0.2$ & $1.8 \pm 0.2$ & 0.11 \\
\hline Height (cm) & $168 \pm 6$ & $167 \pm 8$ & 0.31 \\
\hline Weight (kg) & $71 \pm 13$ & $77 \pm 19$ & 0.11 \\
\hline Diabetes Mellitus $(n, \%)$ & $1 / 322$ & $0 / 27$ & N.A, \\
\hline GFR (mL/min/1.73m²) & $97 \pm 17$ & $97 \pm 16$ & 0.95 \\
\hline Proteinuria (n) & $2 / 322$ & $0 / 27$ & NA \\
\hline Obesity $(n, \%)$ & $134 / 323(41 \%)$ & $15 / 27(56 \%)$ & 0.16 \\
\hline \multicolumn{4}{|l|}{ Index pregnancy } \\
\hline Early-onset PE (n,\%) & 233/318 (72\%) & $18 / 23(67 \%)$ & 0.60 \\
\hline Preterm PE $(n, \%)$ & 268/322 (83\%) & 24/26 (89) & 0.23 \\
\hline SGA (<p10, n, \%) & $87 / 315(28 \%)$ & $7 / 26(26 \%)$ & 0.94 \\
\hline IUFD (n, \%) & $12 / 322(4 \%)$ & $3 / 27(11 \%)$ & 0.07 \\
\hline Perinatal mortality (n, \%) & $17 / 319(5 \%)$ & $2 / 27(7 \%)$ & 0.64 \\
\hline Gestational age at birth (weeks) & $33 \pm 4$ & $31 \pm 4$ & $<0.01$ \\
\hline Primiparous (n, \%) & $263 / 322(81 \%)$ & $24 / 27$ (89\%) & 0.34 \\
\hline Birth weight (g) & $1730 \pm 836$ & $1310 \pm 690$ & $<0.05$ \\
\hline \multicolumn{4}{|l|}{ Cardiac geometry } \\
\hline LVM (g) & $140 \pm 23$ & $146 \pm 21$ & 0.18 \\
\hline LVM index $\left(\mathrm{g} \cdot \mathrm{m}^{-2.7}\right)$ & $34 \pm 6$ & $37 \pm 5$ & 0.05 \\
\hline IVST (mm) & $7.6 \pm 0.7$ & $7.9 \pm 0.7$ & $<0.05$ \\
\hline PWT (mm) & $7.7 \pm 0.6$ & $7.9 \pm 0.5$ & 0.07 \\
\hline RWT & $0.33 \pm 0.03$ & $0.34 \pm 0.03$ & 0.17 \\
\hline LVEDd (mm) & $46 \pm 3$ & $46 \pm 3$ & 0.84 \\
\hline LVESd (mm) & $30 \pm 3$ & $30 \pm 3$ & 0.70 \\
\hline LAD (cm) & $35 \pm 4$ & $37 \pm 3$ & $<0.02$ \\
\hline
\end{tabular}


Table 1 continued

\section{Systolic- and diastolic function}

SysBP $(\mathrm{mmHg})$

$\begin{array}{ccc}115 \pm 10 & 124 \pm 9 & <0.01 \\ 70 \pm 8 & 77 \pm 5 & <0.01 \\ 87 \pm 8 & 94 \pm 7 & <0.01 \\ 68 \pm 13 & 68 \pm 16 & 0.92 \\ 77 \pm 13 & 77 \pm 14 & 0.82 \\ 5.3 \pm 1.0 & 5.3 \pm 0.9 & 0.77 \\ 2.9 \pm 0.5 & 2.9 \pm 0.5 & 0.70 \\ 65 \pm 4 & 65 \pm 5 & 0.76 \\ 1.5 \pm 0.4 & 1.4 \pm 0.3 & 0.10 \\ 1,365 \pm 262 & 1,452 \pm 242 & 0.10 \\ 769 \pm 177 & 799 \pm 186 & 0.40 \\ 100 \pm 16 & 100 \pm 15 & 0.82 \\ 35 \pm 8 & 36 \pm 8 & 0.70\end{array}$

DiasBP $(\mathrm{mmHg})$

MAP $(\mathrm{mmHg})$

Heart Rate (beats $\bullet \mathrm{min}^{-1}$ )

Stroke Volume (ml)

Cardiac Output (L・min $\left.{ }^{-1}\right)$

Cardiac Index $\left(L \bullet \min ^{\prime} \mathrm{m}^{-2}\right)$

Ejection Fraction (\%)

E/A ratio

TPVR (dyn•s•cm-5)

TPVR index $\left(\right.$ dyn $\left.\cdot s \cdot \mathrm{cm}^{-5} \cdot \mathrm{m}^{-2}\right)$

$\operatorname{EDV}(\mathrm{ml})$

$35 \pm 8$

$36 \pm 8$

0.01

0.01

0.01

0.77

0.70

0.76

0.10

0.40

0.82

ESV (ml)

PP: postpartum; IQR: Inter Quartile Range; GFR: Glomerular filtration rate; PE: Preeclampsia; Wk: Weeks; SGA: Small for Gestational Age; IUFD: intrauterine fetal death;; LVM: Left Ventricular Mass; IVST: InterVentricular Septum Thickness; PWT: Posterior Wall Thickness; RWT: Relative Wall Thickness; LVEDd: Left Ventricular End-Diastolic Diameter; LVESd: Left Ventricular End-Systolic Diameter; LAD: Left Atrial Diameter; SysBP: Systolic Blood Pressure; DiasBP: Diastolic Blood Pressure; MAP: Mean Arterial Pressure; TPVR: Total Peripheral Vascular Resistance; EDV: End-Diastolic-Volume; ESV: End-Systolic-Volume;.

Table 2 lists the Hazard Ratios (HR) of cardiac indices determined at postpartum screening and to what extent these indices were associated with the development of hypertension in the course of the follow-up period. Former patients who had developed hypertension differed from their normotensive counterparts by significantly higher postpartum values for LVMi and IVST, SysBP, DiasBP, MAP, Heart Rate and BMI and lower values for E/A ratio. Table 2 also depicts comparable obstetrical characteristics in the two subgroups. 
Table 2: Hazard Rates of Cardiac indices and obstetrical characteristics to the development of hypertension in women with a history of preeclampsia (PE) that where normotensive at postpartum screening.

\begin{tabular}{|c|c|c|}
\hline \multirow[b]{2}{*}{ Indices } & \multicolumn{2}{|c|}{ Univariate } \\
\hline & $\mathrm{HR}(95 \% \mathrm{Cl})$ & p-value \\
\hline \multicolumn{3}{|l|}{ Cardiac geometry } \\
\hline LVM (g) & $1.02(0.99-1.03)$ & 0.06 \\
\hline LVMindex $\left(g \bullet m^{-2.7}\right)$ & $1.11(1.03-1.18)$ & $<0.01$ \\
\hline IVST (mm) & $1.85(1.07-3.20)$ & $<0.05$ \\
\hline PWT (mm) & $1.36(0.67-2.78)$ & 0.40 \\
\hline RWT & $1.03(0.91-1.16)$ & 0.69 \\
\hline LVEDd (mm) & $1.07(0.95-1.21)$ & 0.24 \\
\hline LVESd (mm) & $1.10(0.95-1.27)$ & 0.20 \\
\hline LAD (cm) & $1.12(0.99-1.26)$ & 0.07 \\
\hline \multicolumn{3}{|l|}{ Systolic- and diastolic function } \\
\hline SysBP (mmHg) & $1.07(1.02-1.11)$ & $<0.01$ \\
\hline DiasBP (mmHg) & $1.13(1.06-1.20)$ & $<0.01$ \\
\hline Pre-hypertension & $3.68(1.55-8.74)$ & $<0.01$ \\
\hline MAP (mmHg) & $1.11(1.05-1.18)$ & $<0.01$ \\
\hline Heart Rate (beats•min-1) & $1.05(1.01-1.10)$ & $<0.05$ \\
\hline Stroke Volume (ml) & $0.99(0.96-1.02)$ & 0.60 \\
\hline Caridiac Output (L•min-1) & $1.05(0.73-1.52)$ & 0.80 \\
\hline Cardiac Index $\left(L \cdot \min ^{\prime} \mathrm{m}^{-2}\right)$ & $0.84(0.39-1.78)$ & 0.65 \\
\hline Ejection Fraction (\%) & $0.96(0.87-1.07)$ & 0.51 \\
\hline E/A ratio & $0.22(0.06-0.85)$ & $<0.05$ \\
\hline TPVR (dyn・s・cm-5) & $1.00(1.00-1.00)$ & 0.14 \\
\hline TPVR index (dyn $\left.\cdot \mathrm{s} \cdot \mathrm{cm}^{-5} \cdot \mathrm{m}^{-2}\right)$ & $1.00(0.99-1.00)$ & 0.53 \\
\hline EDV (ml) & $1.01(0.99-1.04)$ & 0.14 \\
\hline ESV (ml) & $1.04(0.99-1.09)$ & 0.17 \\
\hline
\end{tabular}


Table 2 continued

Obstetrical and demographic factors

Age at PP screening (years)

$0.98(0.87-1.09) \quad 0.67$

Body mass index $((\mathrm{kg} / \mathrm{m} 2)$

$1.11(1.04-1.18) \quad<0.01$

Body surface area $\left(\mathrm{m}^{2}\right)$

$1.21(0.98-1.49) \quad 0.08$

Heights (cm)

$0.96(0.90-1.02) \quad 0.17$

Weight $(\mathrm{kg})$

GFR $\left(\mathrm{mL} / \mathrm{min} / 1.73 \mathrm{~m}^{2}\right)$

$1.03(1.00-1.05) \quad<0.05$

Gestational age at birth (weeks)

$1.00(0.98-1.03) \quad 0.83$

$0.99(0.98-1.00) \quad 0.09$

Parity

$0.75(0.30-1.83) \quad 0.52$

Screening months PP (median, IQR)

$1.01(0.99-1.03) \quad 0.55$

Birth weight $(\mathrm{g})$

$1.00(0.99-1.00) \quad 0.12$

SGA $(<p 10)$

$1.13(0.48-2.71) \quad 0.78$

Intrauterine fetal death

$2.19(0.66-7.32) \quad 0.20$

Perinatal mortality

$0.86(0.20-3.65) \quad 0.83$

$\mathrm{PE}$ in next pregnancy

$2.47(0.93-6.54) \quad 0.07$

Early PE

$1.11(0.41-3.02) \quad 0.84$

Preterm PE

Multivariate Backward Stepwise

$1.50(0.35-6.43) \quad 0.58$

$\begin{array}{llc}\text { SysBP }(\mathrm{mmHg}) & 1.02(0.97-1.07) & 0.44 \\ \text { E/A ratio } & 0.36(0.09-1.51) & 0.16 \\ \text { LVMindex }\left(\mathrm{g} \cdot \mathrm{m}^{-2.7}\right) & 1.08(1.01-1.16) & <0.05 \\ \text { DiasBP }(\mathrm{mmHg}) & 1.13(1.06-1.21) & <0.01\end{array}$

LVM: Left Ventricular Mass; IVST: InterVentricular Septum Thickness; PWT: Posterior Wall Thickness; RWT: Relative Wall Thickness; LVEDd: Left Ventricular End Diastolic Diameter; LVESd: Left Ventricular End-Systolic Diameter; LAD: Left Atrial Diameter; SysBP: Systolic Blood Pressure; DiasBP: Diastolic Blood Pressure; MAP: Mean Arterial Pressure; TPVR: Total Peripheral Vascular Resistance; EDV: End-Diastolic-Volume; ESV: End-Systolic-Volume; GFR: Glomerular filtration rate; IQR: Inter Quartile Range; SGA: Small for Gestational Age;

We selected the four most significant variables in the univariate analysis to include in the multivariate analysis. We excluded IVST and MAP and BMI from the multivariate analysis to rule out possible co-linearity. The remaining variables were LVMi, SysBP, DiasBP and E/A ratio. We observed that higher values of LVMi and DiasBP correlated independently with the later development of hypertension. In the subsequent backward stepwise analysis, only the association of LVMi and DiasBP with the development of hypertension remained significant (HR of 1.08 (95\% Cl 1.01-1.16) $\mathrm{p}<0.05$ for LVMi, and a HR of 1.13 (95\% Cl 1.06-1.21) p<0.01 for DiasBP).

Subsequently, subjects were stratified according to the presence of the highest-riskquartile (Q) values for LVMi $\left(\geq 37 \mathrm{~g} / \mathrm{m}^{2.7}\right)$ and DiasBP $(\geq 75 \mathrm{mmHg})$ ). Kaplan Meier curves (fig.2) revealed a potential threshold effect for DiasBP en LVMi in the upper Q. After a follow-up time of 10 years, former patients with DiasBP in the $4^{\text {th }} \mathrm{Q}$ had a cumulative probability of almost $20 \%$ to develop hypertension, which is significantly higher than the probability of $10 \%$ of the former patients with a DiasBP below the $4^{\text {th }} \mathrm{Q}$ (fig.2a). In analogy, women with LVMi in the $4^{\text {th }}$ quartile showed a cumulative probability of over $20 \%$ to develop hypertension within 10 years, which was also significantly higher than 
that in former patients with an LVMi below the $4^{\text {th }} \mathrm{Q}$, who had a cumulative probability of only $5 \%$ to develop hypertension (fig. $2 \mathrm{~b}$ ). Furthermore, fig. $2 \mathrm{c}$ shows that when either DiasBP or LVMi are in the $4^{\text {th }} \mathrm{Q}$, the cumulative probability of developing hypertension within 10 years is about $20 \%$, clearly higher than the close to zero cumulative probability in former patients with neither of these 2 indices in the $4^{\text {th }} \mathrm{Q}$. Finally, fig.2d shows that when both indices are in the $4^{\text {th }} \mathrm{Q}$, the cumulative risk of developing hypertension within 6 years is over $20 \%$ compared to $5 \%$ when one or none of the indices is in the $4^{\text {th }} \mathrm{Q}$.
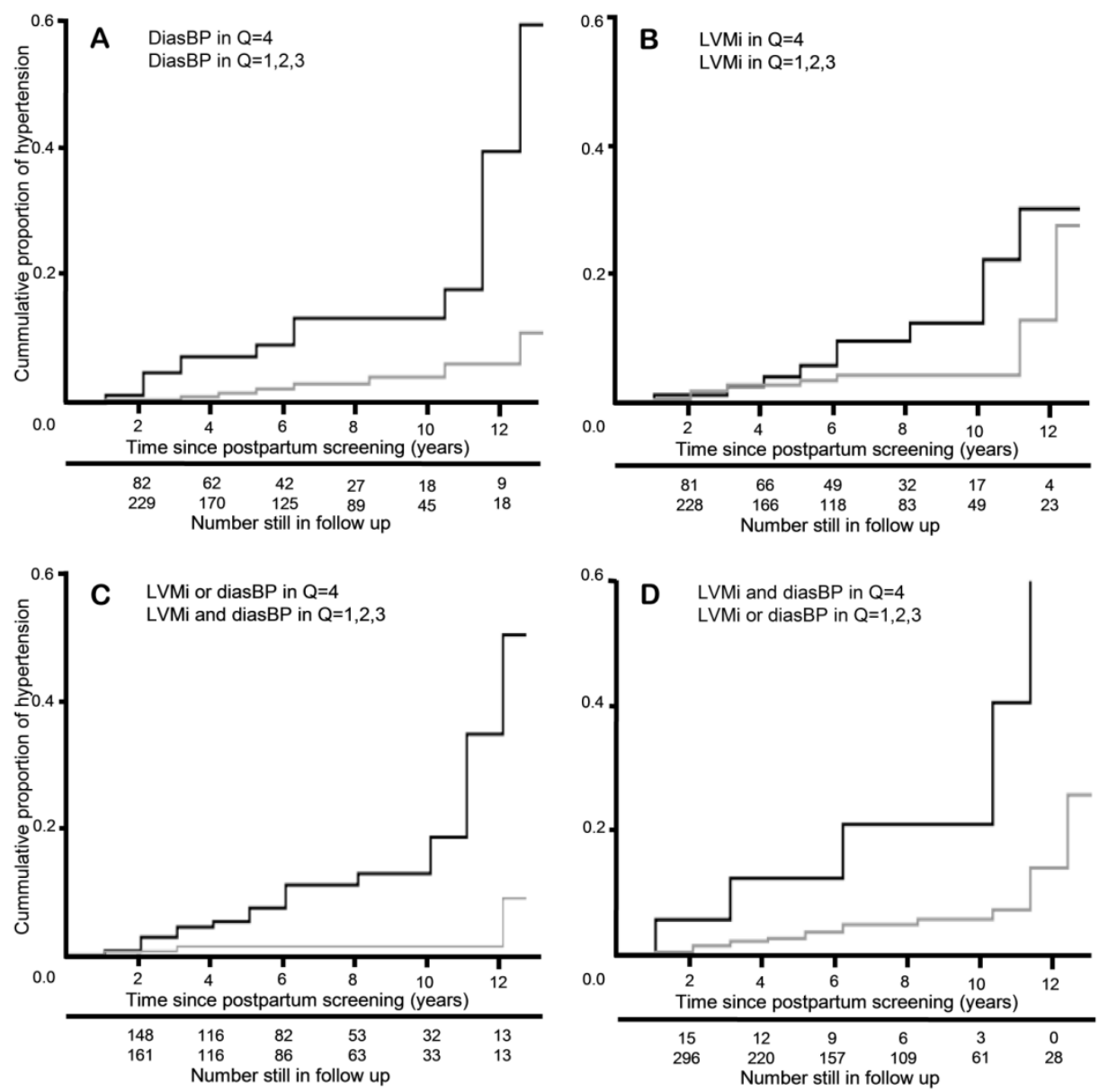

Figure 2: Kaplan Meier curve of the cumulative proportion of normotensive formerly preeclamptic patients that had developed hypertension. Groups are stratified by the upper quartile of A) DiasBP (Diastolic Blood Pressure $\geq 75 \mathrm{mmHg}$ ) B) LVMi (Left ventricular mass index $\geq 37 \mathrm{~g} / \mathrm{m}^{2.7}$ ); C) either one of them D) and both together. The log rank test was used to test if the Kaplan Meier curves were significantly different. The $p$-values were respectively $<0.01,<0.05,<0.01$ and $<0.01$. 
Table 3 shows the demographic and obstetric characteristics of the normotensive former patients who did (responders) and did not respond (non-responders) to at least one completed questionnaire. Both groups were comparable with respect to important prognostic factors. Only the percentage of intrauterine fetal death was borderline significantly higher in the group of non-responders compared to the group of responders ( $9 \%$ versus $4 \%$ respectively, $p=0.06$ ).

Table 3: Demography and obstetrical history of the responder and non-responder subgroup, analyzed with the independent t-test or Chi-square. Data are presented as mean with Standard Deviation (SD), unless stated otherwise.

\begin{tabular}{lccc}
\hline Indices & $\begin{array}{c}\text { Responders } \\
\mathrm{N}=349\end{array}$ & $\begin{array}{c}\text { Non-responders } \\
\mathrm{N}=128\end{array}$ & $\mathrm{p}$-value \\
\hline Age at PP screening (years) & $31 \pm 4$ & $31 \pm 5$ & 0.38 \\
Months PP (median, IQR) & $9(6-19)$ & $9(6-18)$ & 0.54 \\
Body mass index (kg/m2) & $25 \pm 5$ & $26 \pm 6$ & 0.37 \\
Index pregnancy & & & \\
Early PE (n,\%) & $251 / 342(73 \%)$ & $84 / 122(67 \%)$ & 0.31 \\
Late PE (n,\%) & $91 / 342(27 \%)$ & $38 / 122(30 \%)$ & 0.31 \\
SGA (<p10, n, \%) & $94 / 342(28 \%)$ & $33 / 121(26 \%)$ & 0.95 \\
IUFD (n, \%) & $15 / 349(4 \%)$ & $11 / 124(9 \%)$ & 0.06 \\
Perinatal mortality (n, \%) & $19(5 \%)$ & $9(7 \%)$ & 0.48 \\
Gestational age at birth (weeks) & $33 \pm 4$ & $33 \pm 4$ & 0.12 \\
Primiparous (n, \%) & $288(82 \%)$ & $105(83 \%)$ & 0.88 \\
Birth weight (g) & $1,698 \pm 833$ & $1,839 \pm 920$ & 0.15 \\
\hline
\end{tabular}

IQR: interquartile-range; PE: Preeclampsia; PP: Postpartum; SGA: Small for Gestational Age; IUFD: Intrauterine Fetal Death.

\section{Discussion}

This large and comprehensive long-term study of the postnatal cardiac and hypertensive consequences of preeclampsia highlights the previously underestimated cardiac burden of this pregnancy disorder.

Initially normotensive formerly preeclamptic women destined to develop hypertension within the next 12 years, differ from their counterparts who remain normotensive throughout this period, by increased LVMi and decreased cardiac diastolic function at the time of postpartum screening.

In a normal pregnancy left-ventricular mass and dimensions increase. ${ }^{4}$ This eccentric hypertrophy is a healthy physiological response that reverses within few weeks postpartum. ${ }^{4}$ In contrast, during PE, women show more pronounced increase in LVM, but with less pronounced LV widening. ${ }^{4} 13$ This concentric hypertrophy develops secondary to the increased LV workload that accompanies the elevated blood pressure. ${ }^{14}$ For myocyte growth to support an increased biomechanical load, it must be accompanied by coordinated increases in the surrounding architecture of connective tissue and ground substance that primary consists of collagen. This leads to increased 
cardiac stiffness, a form of remodeling that reduces the mechanical efficiency of the heart. Hence, it is associated with higher cardiac oxygen demands. Concentric cardiac remodeling is also accompanied by a decline in diastolic function, reflected in larger values for left atrial diameter and circulating atrial natriuretic peptide levels. ${ }^{15}$

There is evidence for persisting cardiovascular impairment in the immediate postpartum period. ${ }^{4,16}$ LV hypertrophy in acute PE may have been an adaptive response to minimize wall stress with the sudden development of hypertension in PE. ${ }^{4,17}$ Residual adverse LV altered remodeling might sustain or even progress if high BP persists. ${ }^{18}$ Despite the profound effects of PE on the ultrasonic cardiac indices, only few studies explored cardiac function and geometry for an extended postpartum period. It has been reported that normotensive formerly PE women show impaired systolic function 13-18 years postpartum even though SV and CO are preserved. ${ }^{5,19}$ Available postpartum data are not completely consistent, most likely as a consequence of the heterogeneous pathogenesis and clinical presentation of PE. ${ }^{20}$

Recent guidelines have highlighted the importance of asymptomatic LV dysfunction and hypertrophy in the risk stratification for cardiac disease and heart failure. ${ }^{21}$ One year postpartum, mild asymptomatic heart failure is mainly present in women who experienced early-onset PE. ${ }^{6}$ These former patients are at increased risk of atrial arrhythmias. ${ }^{22}$ The progression from asymptomatic to symptomatic heart failure is associated with a 5-fold increase in the risk of related death, which can be counterbalanced by timely therapeutic intervention. ${ }^{18}$ Moreover, even a mildly raised BP is associated with increased LVM. ${ }^{23}$ Even in a hypertensive patient, LVM appears to be reversible when BP is lowered at an early stage. ${ }^{23}$ Timely identification of women with persisting postpartum structural cardiac abnormalities may contribute to the secondary prevention of more progressed CVD.

On the one hand, we observed a high percentage of women who remained or became hypertensive at time of screening (28\%). On the other hand, an additional $8 \%$ of initially normotensive women at postpartum screening developed hypertension in later years. The total prevalence of hypertension in our cohort is $36 \%$, a figure which is comparable with reported data ${ }^{6}$ and markedly higher than the 3 to $6 \%$ prevalence of hypertension in Dutch women between 20-39 years. ${ }^{24}$ The high prevalence of chronic hypertension in our cohort could be related to the high rate of early-onset PE and preterm PE (73\% and $84 \%$ respectively), known to be associated with high risk of developing chronic hypertension and CVD. ${ }^{2,6}$ However we did not find a significant association of early or preterm PE with the development of later hypertension in initially normotensive women. Perhaps early/preterm PE plays a bigger role in direct postpartum hypertension than in developing later hypertension. Hypertension in the early postpartum period may still normalize over the years, as 14 out of 74 initially hypertensive women did not use any antihypertensive medication during follow-up.

The incidence of Diabetes mellitus, obtained by self-report, is lower than one would expect in this population. Because we excluded all women that were hypertensive at baseline, we might also have excluded those women that are glucose intolerant since hypertension and glucose intolerance often co-exist.

Our large and comprehensive long-term study of the postnatal cardiac and hypertensive consequence of $\mathrm{PE}$, highlighting the significant cardiac burden of this pregnancy disorder, has several limitations. First, we estimated the incidence of 
hypertension by self-report. On the one hand, this might lead to underestimation of the prevalence of hypertension in subjects who do not regularly check their BP. On the other hand, healthy women are less likely to respond to the questionnaire, which could lead to overestimation. Though these kinds of bias often accompanies such retrospective analyses, the effect might have been minimal since in our study population, medical, demographic, and obstetric characteristics were comparable in responders and non-responders.

Chronic hypertension may originate from altered circulatory function or adverse metabolic factors jeopardizing endothelial function. On the one hand, $74 \%$ of the normotensive women, who went on to develop chronic hypertension, fulfilled the criteria of pre-hypertension. Pre-hypertension is defined as a SysBP ranging from 120 to $139 \mathrm{mmHg}$ and/or DiasBP ranging from 80 to $89 \mathrm{mmHg} .{ }^{25}$ Pre-hypertension is associated with increased CO, TPVRi and LVMi. ${ }^{26}$ Persistent pre-hypertension accelerates the development of hypertrophy and diastolic dysfunction of the heart. ${ }^{27}$ Moreover, it is associated with an increased risk of myocardial infarction and coronary arterial disease (relative risk of $3.5(95 \% \mathrm{Cl}, 1.6-7.5)$ and $1.7(95 \% \mathrm{Cl}, 1.2-2.4)$, respectively). ${ }^{28}$ On the other hand, seemingly healthy women may demonstrate unfavorable metabolic and vascular changes. ${ }^{29}$ Thus, subclinical risk factors can make women more susceptible to slightly increased BP within the normotensive range, which may manifest as subclinical increase in measures of left ventricular geometry and decreased diastolic function. As such, the Hypertension Writing Group suggests that, although measuring BP remains one of the most important cornerstones of the clinical assessments of patients at risk of hypertension-related CVD, hypertension cannot be classified solely on the basis of discrete blood pressure thresholds and that the levels of BP must be evaluated in the context of the total cardiovascular risk assessment. ${ }^{30}, 25$

Our results are in line with our expectations and could also be true in other nonpreeclamptic populations. However, cardiovascular findings related to PE should not be generalized to other subpopulations or the other way around for the following reasons.

First, one in three women with a history of PE will develop chronic hypertension within 12 years after delivery. Thus the incidence of chronic hypertension is much higher than in the general population ( $36 \%$ Vs $6 \%$ ). Second, formerly PE patients are seemingly healthy and belong to a fertile and much younger subpopulation (30-40 years) then major other populations with an increased CV risk.

Third, it is debated whether preeclampsia itself is a risk factor for CVD or whether there are additional, more classic risk factors that add on to the risk. There is increasing evidence that PE and CVD do share the same risk factors. Part of these factors can be modified if identified at an early stage. However, it is still not clear to what extend the classic risk factors really add on to the risk of CVD and to what extend modification of these factors decreases the risk. 


\section{Conclusion}

The development of remote chronic hypertension in initially normotensive formerly preeclamptic women is preceded by increased left ventricular mass index and diastolic blood pressure at postpartum screening.

\section{Perspectives}

Though formerly PE women are at significantly increased risk for premature CVD and despite the fact that they develop CVD at a young age, it's often only recognized at a progressed stage of their disease. Therefore it is needed to increase the awareness of obstetricians and cardiovascular risk assessors to the early recognisable risk factors that, when identified, can be addressed and perhaps modified.

\section{Novelty and Significance}

What Is New?

- Increased diastolic blood pressure and LVMi in formerly preeclamptic women might indicate those at increased hypertensive risk.

- $\quad 76 \%$ of the normotensive formerly preeclamptic women, who went on to develop chronic hypertension, fulfilled the criteria of pre-hypertension at postpartum screening.

What Is Relevant?

- $\quad$ Preeclampsia is associated with a 4 fold increased risk of chronic hypertension in women.

- Despite the increased risk, it is still not possible to identify at an early stage those at risk.

- As timely antihypertensive therapy may reduce CVD and mortality, these women may benefit from early detection.

\section{Summary:}

- $\quad$ These findings indicate that the development of remote chronic hypertension in initially normotensive formerly preeclamptic women is preceded by increased left ventricular mass index and diastolic blood pressure at postpartum screening.

\section{Acknowledgements}

We would like to thank Inez Schreij, Timo Ekhart and Nienke Heemskerk for their contribution in the data collection. 


\section{References}

1. Sibai B, Dekker G, Kupferminc M. Pre-eclampsia. Lancet. 2005;365:785-799.

2. Bellamy L, Casas JP, Hingorani AD, Williams DJ. Pre-eclampsia and risk of cardiovascular disease and cancer in later life: systematic review and metaanalysis. Bmj. 2007;335:974.

3. Melchiorre K, Thilaganathan B. Maternal cardiac function in preeclampsia. Curr Opin Obstet Gynecol. 2011;23:440-447.

4. Simmons LA, Gillin AG, Jeremy RW. Structural and functional changes in left ventricle during normotensive and preeclamptic pregnancy. Am J Physiol Heart Circ Physiol. 2002;283:H1627-1633.

5. Evans CS, Gooch L, Flotta D, Lykins D, Powers RW, Landsittel D, Roberts JM, Shroff SG. Cardiovascular system during the postpartum state in women with a history of preeclampsia. Hypertension.58:57-62.

6. Melchiorre K, Sutherland GR, Liberati M, Thilaganathan B. Preeclampsia is associated with persistent postpartum cardiovascular impairment. Hypertension. 2011;58:709-715.

7. Report of the National High Blood Pressure Education Program Working Group on High Blood Pressure in Pregnancy. Am J Obstet Gynecol. 2000;183:S1-S22.

8. DuBois D, DuBois EF. A formula to estimate the approximate surface area if height and weight be known. Arch. Int. Med. 1916;17:863-871.

9. Sahn DJ, DeMaria A, Kisslo J, Weyman A. Recommendations regarding quantitation in M-mode echocardiography: results of a survey of echocardiographic measurements. Circulation. 1978;58:1072-1083.

10. Devereux RB, Casale PN, Kligfield P, Eisenberg RR, Miller D, Campo E, Alonso DR. Performance of primary and derived M-mode echocardiographic measurements for detection of left ventricular hypertrophy in necropsied subjects and in patients with systemic hypertension, mitral regurgitation and dilated cardiomyopathy. Am J Cardiol. 1986;57:1388-1393.

11. de Simone G, Daniels SR, Devereux RB, Meyer RA, Roman MJ, de Divitiis O, Alderman $\mathrm{MH}$. Left ventricular mass and body size in normotensive children and adults: assessment of allometric relations and impact of overweight. J Am Coll Cardiol. 1992;20:1251-1260.

12. Teichholz LE, Kreulen T, Herman MV, Gorlin R. Problems in echocardiographic volume determinations: echocardiographic-angiographic correlations in the presence of absence of asynergy. Am J Cardiol. 1976;37:7-11.

13. Valensise H, Vasapollo B, Gagliardi G, Novelli GP. Early and late preeclampsia: two different maternal hemodynamic states in the latent phase of the disease. Hypertension. 2008;52:873-880.

14. Vasapollo B, Novelli GP, Valensise $H$. Total vascular resistance and left ventricular morphology as screening tools for complications in pregnancy. Hypertension. 2008;51:1020-1026.

15. Tihtonen KM, Koobi T, Vuolteenaho O, Huhtala HS, Uotila JT. Natriuretic peptides and hemodynamics in preeclampsia. Am J Obstet Gynecol. 2007;196:328 e321327. 
16. Rafik Hamad R, Larsson A, Pernow J, Bremme K, Eriksson MJ. Assessment of left ventricular structure and function in preeclampsia by echocardiography and cardiovascular biomarkers. J Hypertens. 2009;27:2257-2264.

17. Melchiorre K, Sutherland GR, Baltabaeva A, Liberati M, Thilaganathan B. Maternal cardiac dysfunction and remodeling in women with preeclampsia at term. Hypertension. 2011;57:85-93.

18. Ammar KA, Jacobsen SJ, Mahoney DW, Kors JA, Redfield MM, Burnett JC, Jr., Rodeheffer RJ. Prevalence and prognostic significance of heart failure stages: application of the American College of Cardiology/American Heart Association heart failure staging criteria in the community. Circulation. 2007;115:1563-1570.

19. Strobl I, Windbichler G, Strasak A, Weiskopf-Schwendinger V, Schweigmann U, Ramoni $A$, Scheier $M$. Left ventricular function many years after recovery from pre-eclampsia. BJOG. 2011;118:76-83.

20. Vatten LJ, Skjaerven R. Is pre-eclampsia more than one disease? Bjog. 2004;111:298-302.

21. Jessup M, Abraham WT, Casey DE, Feldman AM, Francis GS, Ganiats TG, Konstam MA, Mancini DM, Rahko PS, Silver MA, Stevenson LW, Yancy CW. 2009 focused update: ACCF/AHA Guidelines for the Diagnosis and Management of Heart Failure in Adults: a report of the American College of Cardiology Foundation/American Heart Association Task Force on Practice Guidelines: developed in collaboration with the International Society for Heart and Lung Transplantation. Circulation. 2009;119:1977-2016.

22. Ray JG, Schull MJ, Kingdom JC, Vermeulen MJ. Heart failure and dysrhythmias after maternal placental syndromes: HAD MPS Study. Heart. 2012;98:1136-1141.

23. Lauer MS, Anderson KM, Levy D. Influence of contemporary versus 30-year blood pressure levels on left ventricular mass and geometry: the Framingham Heart Study. J Am Coll Cardiol. 1991;18:1287-1294.

24. Blokstra A SH, Bueno de Mesquita HB, Seidell JC, Verschuren WMM. Monitoring van Risicofactoren en Gezondheid in Nederland (MORGENproject) 19931997. Leefstijl en risicofactoren: prevalenties en trends. Bilthoven: RIVM, 2005. RIVM rapport 263200008.

25. Giles TD, Materson BJ, Cohn JN, Kostis JB. Definition and classification of hypertension: an update. J Clin Hypertens (Greenwich). 2009;11:611-614.

26. Drukteinis JS, Roman MJ, Fabsitz RR, Lee ET, Best LG, Russell M, Devereux RB. Cardiac and systemic hemodynamic characteristics of hypertension and prehypertension in adolescents and young adults: the Strong Heart Study. Circulation. 2007;115:221-227.

27. Markus MR, Stritzke J, Lieb W, Mayer B, Luchner A, Doring A, Keil U, Hense HW, Schunkert $H$. Implications of persistent prehypertension for ageing-related changes in left ventricular geometry and function: the MONICA/KORA Augsburg study. J Hypertens. 2008;26:2040-2049.

28. Qureshi Al, Suri MF, Kirmani JF, Divani AA, Mohammad Y. Is prehypertension a risk factor for cardiovascular diseases? Stroke. 2005;36:1859-1863.

29. Garovic VD, Hayman SR. Hypertension in pregnancy: an emerging risk factor for cardiovascular disease. Nat Clin Pract Nephrol. 2007;3:613-622. 
30. Chobanian AV, Bakris GL, Black HR, Cushman WC, Green LA, Izzo JL, Jr., Jones DW, Materson BJ, Oparil S, Wright JT, Jr., Roccella EJ. Seventh report of the Joint National Committee on Prevention, Detection, Evaluation, and Treatment of High Blood Pressure. Hypertension. 2003;42:1206-1252. 


\section{Chapter 5}

\section{Long-term risk to develop hypertension in}

formerly preeclamptic women:

\section{A longitudinal pilot study}

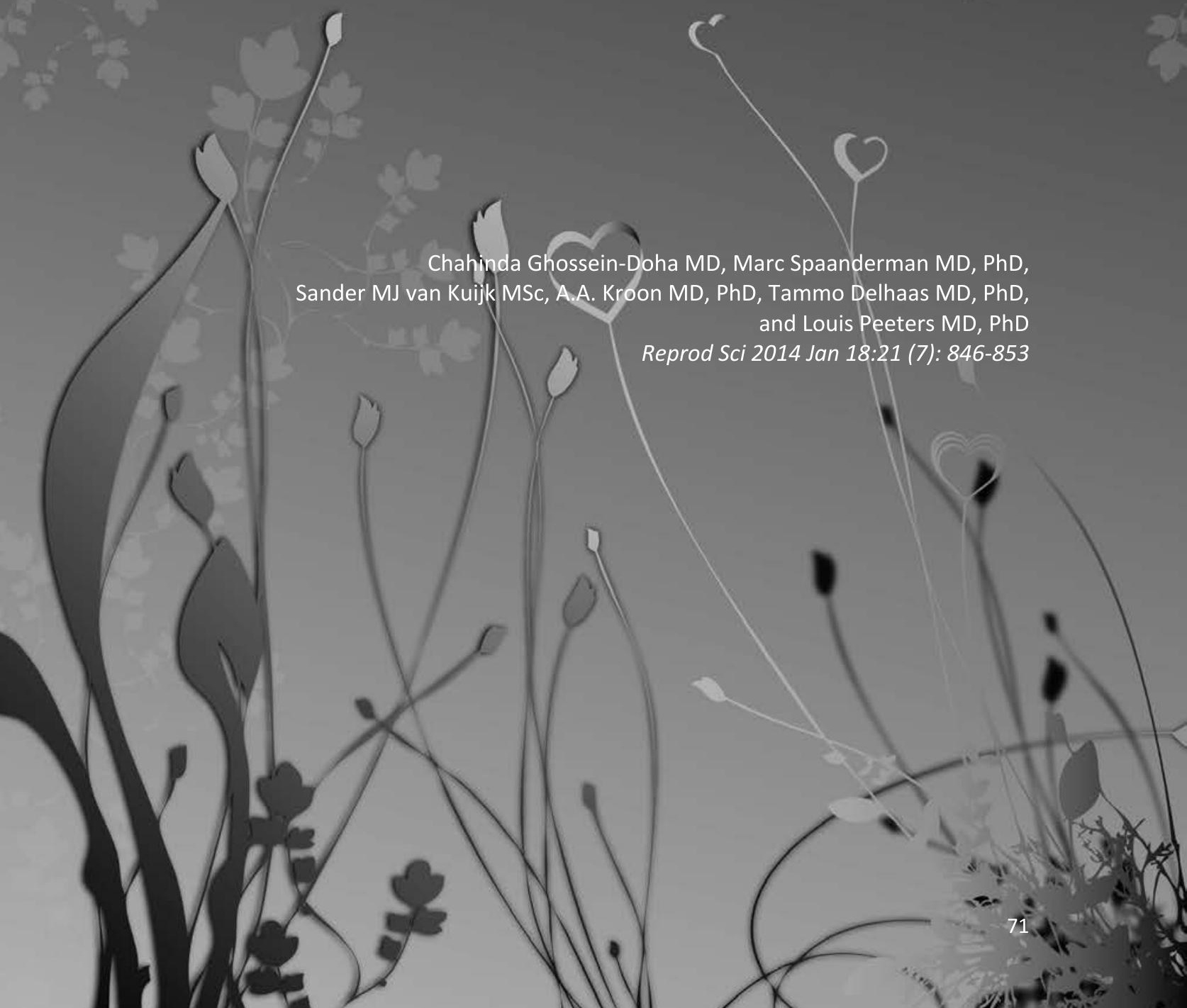




\begin{abstract}
Introduction: Formerly preeclamptic women develop chronic hypertension 4 times more often than healthy parous controls. Women, destined to develop remote chronic hypertension, had increased left-ventricular mass index (LVMI) and diastolic blood pressure (BP) prior to the onset of hypertension as compared to those remaining normotensive. However, longitudinal data on the progress of this increased LVMI in women destined to develop hypertension is lacking.
\end{abstract}

Methods: We included 20 formerly preeclamptic women and 8 parous controls. At both 1 and 14 years postpartum (pp.), we performed cardiac-ultrasound and determined circulating levels of the metabolic-syndrome variables. Fourteen years pp., 7 of these former patients (35\%) had developed chronic hypertension. We compared these 7 former patients with both the 13 former patients who remained normotensive and the 8 parous controls, using the Mann-Whitney-U test and Kruskal-Wallis analysis.

Results: Hypertensive formerly-preeclamptic women differed from their normotensive counterparts by a higher incidence of early-onset preeclampsia in their index pregnancy and a higher rate of recurrence in next pregnancies. At 1-year pp., they also had high/normal BP and higher fasting insulin-levels. At 14 years pp., the relative leftventricular wall thickness was higher and the E/A ratio lower, in the hypertensive group relative to those remaining normotensive.

Conclusion: Formerly preeclamptic women are at increased risk of developing chronic hypertension, when 1) the preeclampsia in the index pregnancy had an early-onset and/or recurred in next pregnancies, and 2) the 1-year pp. BP was high-normal. We also noticed that at 14 years pp., the hypertensive group showed signs of concentric left-ventricular remodeling along with a decreased E/A ratio. 


\section{Introduction}

Preeclampsia (PE), a hypertensive pregnancy disorder complicating 3-5\% of all pregnancies in the Western world, is a major cause of perinatal morbidity and mortality. ${ }^{1}$ Although a link between PE and future chronic hypertension has already been suggested almost 50 years ago ${ }^{2}$, it was only recently, that PE has been generally accepted not only to predispose to chronic hypertension, but also to premature cardiovascular disease (CVD). ${ }^{3}$ PE and CVD share many risk conditions such as diabetes, obesity, and preexistent hypertension. Still, it is unclear whether PE itself, underlying risk conditions, or a combination contributes to future hypertension and cardiovascular disease.

Compared to parous controls, formerly preeclamptic women (exPE) have a fourfold higher risk to develop chronic hypertension. ${ }^{4}$ From the available epidemiological data it is not possible to determine, whether this higher risk applies to all former patients or to a subgroup with specific underlying risk factors or latent disorders. This is relevant as these epidemiologic studies do not stratify for different pathogenetic pathways preceding the onset of PE. ${ }^{5}$ Previously, we found a larger left ventricular mass index and a higher incidence of high-normal diastolic blood pressures in formerly preeclamptic women, normotensive at the 1-year postpartum (pp.) screening, who eventually developed chronic hypertension. ${ }^{6}$ However, the design of that study was cross-sectional with follow-up information being obtained by biennial questionnaires. These do not provide information on cardiac remodeling after the onset of hypertension. The latter is important as only half of the patients with chronic hypertension develop left-ventricular remodeling, which may be either concentric or eccentric depending on whether the hypertension is triggered by pressure or volume overload, resp. ${ }^{7,8}$ In addition, it is not known whether left-ventricular remodeling develops similarly in former PE women who are much younger than hypertensive subjects in the general population. Moreover, in the previous study we did not include information on the metabolic syndrome, which is associated with both PE and chronic hypertension. 9

Therefore, this explorative pilot study was designed to investigate whether seeminglyhealthy, formerly-preeclamptic women destined to develop hypertension in a 14years' follow-up period since the PE pregnancy 1) show accelerated cardiac remodeling in this time interval and 2) show increased metabolic syndrome risk factors, already present at 1-year pp. To this end, we performed cardiac ultrasound and measured circulating levels of the variables contributing to the metabolic syndrome at 1-year and 14 -years pp. in 20 formerly-preeclamptic women and in 8 healthy parous controls. The observations at 14-years pp. were used to subdivide the 20 former patients into 2 subgroups depending on whether (HT-exPE) or not (NT-exPE) chronic hypertension had developed. 


\section{Patients and Methods}

\section{Study population}

Before the start of the study, the hospital's Medical-Ethical Committee approved the study protocol (MEC 08-2-130). We defined PE according to the criteria of the International Society for the Study of Hypertension in Pregnancy. ${ }^{11}$ PE was considered "early-onset", when the disorder was diagnosed before 34 weeks pregnancy. We considered newborns to be small-for-gestational-age (SGA) if their birthweight was below the $5^{\text {th }}$ centile according to the birthweight reference curves of the Perinatal Registry in the Netherlands. ${ }^{12}$ We sent an invitation to the women, who had participated in a previous study between 1996 and 1999, ${ }^{13}$ to take part in this followup study. The study population in that previous report only consisted of Caucasian women, with 10 women being healthy normotensive controls and 39 being primiparous after a preeclamptic pregnancy. At that time, women with a history of preeclampsia were recruited at the outpatient clinic at postpartum follow-up. Most of them experienced a severe form of preeclampsia, either early-onset preeclampsia or preeclampsia complicated by fetal growth restriction or fetal demise. Parous controls were recruited by advertisement. We invited the 10 parous controls and 33 former patients for the 14 year pp. follow-up measurement who had neither pre-existing renal disease nor persistent postpartum hypertension. Three of these former patients were lost to follow-up, one woman had died of pulmonary embolism and one was undergoing chemotherapy. Furthermore, 8 former patients and 2 controls declined participation because of lack of time and/or emotional reasons. Eventually, 20 former patients and 8 parous controls (CONTR) agreed to participate in this study.

We divided the former patients into 2 subgroups based on whether or not they had developed chronic hypertension at the 14 years pp. measurement session. One subgroup consisted of 13 former patients who were still normotensive at the 14 years pp. measurement (NT-exPE), whereas the other subgroup consisted of 7 former patients who had developed chronic hypertension by then (HT-exPE), requiring antihypertensive medication. We diagnosed chronic hypertension on the basis of a blood pressure $\geq 140 / 90 \mathrm{mmHg}$ measured by standard criteria (see below). Prehypertension was defined by systolic and diastolic blood pressures ranging from 120 to 139, and/or from 80 to $89 \mathrm{mmHg}$, respectively. Metabolic syndrome (MetS) was diagnosed according to the National Cholesterol Education Program-Adult Treatment Panel III criteria (NCEP-ATP III). ${ }^{14}$

\section{Measurements}

The participants underwent the same set of measurements at 1 and 14 year pp. We performed both measurements sessions in the mid-follicular phase of the menstrual cycle (day $5 \pm 2$ ). Participants used a standard sodium diet $\left(100 \mathrm{mmol}\right.$ sodium $\cdot$ day $^{-1}$ ) starting 1 week prior to measurement. Antihypertensive drugs were discontinued 2 weeks earlier. From 10 hours prior to measurements, participants refrained from smoking, eating and drinking caffeine- or alcohol-containing beverages. 
The measurement session started at 8:00 am in a temperature-controlled room $( \pm 24$ oC), with as little as possible external disturbances. Participants were lying on their back on a comfortable bed throughout the measurement session. We measured blood pressure on two consecutive occasions using an oscillometric device ((Dinamap Vital Signs Monitor 1846; Critikon Company LLC, Tampa, Fla). First we recorded blood pressure for 30 minutes at 3-min. intervals after $30 \mathrm{~min}$. acclimatization in these standardized environmental conditions. In all women enrolled in the "hypertensive" subgroup, we confirmed the diagnosis. That is to say, they all had a median systolic and/or diastolic blood pressures in excess of 140 and/or $90 \mathrm{mmHg}$, resp. After the blood pressure recording, we sampled fasting blood for the later measurement of glucose $\left(\mathrm{mmol} \cdot \mathrm{L}^{-1}\right)$, insulin $\left(\mathrm{mU} \cdot \mathrm{L}^{-1}\right)$, Low Density Lipoprotein $\left(\mathrm{LDL}, \mathrm{mmol} \cdot \mathrm{L}^{-1}\right)$, High Density Lipoprotein $\left(\mathrm{HDL}, \mathrm{mmol} \cdot \mathrm{L}^{-1}\right)$, triglycerides $\left(\mathrm{mmol} \cdot \mathrm{L}^{-1}\right)$, and total cholesterol $\left(\mathrm{mmol} \cdot \mathrm{L}^{-1}\right.$ ) using standard laboratory techniques at the laboratory of the University Medical Center Maastricht. We estimated the degree of insulin resistance using the Homeostatic Model Assessment (HOMA) by the following formula: (glucose ( $\mathrm{mmol}^{-1} \mathrm{~L}^{-1}$ ) $\mathrm{x}$ insulin $\left.\left(\mathrm{mU} \cdot \mathrm{I}^{-1}\right)\right) / 22.5{ }^{15}$ Body mass index $\left(\mathrm{BMI}, \mathrm{kg} \cdot \mathrm{m}^{-2}\right.$ ) was calculated by dividing body weight in $\mathrm{kg}$ by squared length in meters. Overweight was defined as BMI between 25 and $30 \mathrm{~kg} \cdot \mathrm{m}^{-2}$. Body surface area (BSA) was calculated as follows: BSA $\left(\mathrm{m}^{2}\right)$ $=0.007184 \cdot$ height $(\mathrm{cm})^{0.725} \cdot$ weight $(\mathrm{kg})^{0.425} \cdot 16$

We assessed cardiac function with the participant in dorsal recumbence using a phased-array echocardiographic Doppler system (Hewlett-Packard Sonos 2000 and 2500; Hewlett-Packard Company, Palo Alto, USA) as detailed previously. ${ }^{13}$ All data were analyzed offline using specific software (Excelera, Philips, The Netherlands).

By M-mode in the parasternal long-axis view, we measured left-ventricular enddiastolic and end-systolic diameters (LVEDd, LVESd, $\mathrm{mm}$ ), and the end-diastolic thickness of both the inter-ventricular septum (IVST, $\mathrm{mm}$ ) and the posterior wall (PWT, $\mathrm{mm}$ ). We used the Devereux-formula ${ }^{17}$ to estimate left-ventricular mass (LVM) both as an absolute figure $(\mathrm{g})$ and indexed for height in meters ${ }^{2.7} .{ }^{18}$ We calculated relative wall thickness (RWT) as follows: RWT = [IVST+PWT] / LVEDd. The heart rate (HR, beats. $\mathrm{min}^{-}$ $\left.{ }^{1}\right)$ was obtained by taking the reciprocal of the mean of 5 consecutive RR-intervals on the electrocardiogram (ECG) multiplied by 60 . We estimated the mean aortic Velocity Time Integral (VTI) by averaging the outer edge tracings of 5 consecutive Continuous Wave (CW) Doppler registrations of the aortic flow at the level of the aortic valve. By taking the product of VTI and the cross-sectional area at the level of the aortic annulus in the parasternal long axis view, we obtained stroke volume (SV, $\mathrm{mL}$ ). Finally, cardiac output $\left(\mathrm{CO}, \mathrm{L} \cdot \mathrm{min}^{-1}\right)$ was obtained by multiplying SV with HR. During cardiac ultrasound, we repeated the blood pressure (BP) measurement (in triplicate) using the same semi-automatic oscillometric device as used before. The (median) values of this 2nd set of measurements differed little and inconsistently from the ones obtained earlier, but nevertheless were reported in the tables and used for statistical analysis. By measuring the transmitral flow pattern by pulsed-wave (PW) Doppler echocardiography in the apical 4-chamber view, we derived the E/A ratio, which provides a crude estimate for diastolic function and corresponds with the ratio of peak mitral flow velocity during early diastole (E) and that during atrial contraction (A). Doppler-derived indices were averaged over 5 consecutive cardiac cycles. 
The PW Doppler sample volume $(5 \mathrm{~mm})$ was carefully positioned at the tip of the mitral valve leaflets. The sweep rate was set at $50 \mathrm{~mm} \cdot \mathrm{s}^{-1}$.

\section{Statistical analysis}

We compared the three groups by Kruskal-Wallis analysis, and the two former-patients subgroups using the Mann-Whitney- $U$ test. Categorical data were analyzed by the $X^{2}$ test if at least 5 cases were present in each of the 3 groups and by the Fisher exact test if one of the groups contained less than 5 cases. Of the glucose, insulin and cholesterol data determined at 1-year pp., 4\%, 30\% and 48\%, respectively, were missing. At 14 year pp., none of the measurements was missing. We used regression to impute missing values as limiting the analysis to complete datasets only would have led to loss of precision or biased results. ${ }^{19}$ The imputation step and all analyses were performed using SPSS version 17.0. Data are presented as median with inter-quartile range (IQR) unless stated otherwise. We considered a p-value below 0.05 (after correction for multiple testing) to be statistically significant.

\section{Results}

Seven (35\%) former patients had developed chronic hypertension at 14 years pp. compared to only one in the control group (12.5\%). Table 1 lists the demography and pregnancy outcome in our study population. HT-exPE differed from NT-exPE by having suffered twice as often early-onset PE in their index pregnancy (100\% vs. 46\%, $p<0.05)$, giving birth at an earlier gestational age to a child with a lower birthweight. Moreover, HT-exPE suffered more often of recurrent hypertensive pregnancy disorders than NTexPE ( $86 \%$ vs. $22 \%)$.

Table 2 lists blood pressures and various metabolic variables in both subgroups of former patients and the controls at 1 and 14 years $\mathrm{pp}$. Already at 1 year pp., HT-exPE differed from NT-exPE by a higher systolic, diastolic, and mean arterial BP, and from the control group by higher diastolic and mean arterial BP. We observed neither at 1 year nor at 14 years pp. consistent differences in glucose and insulin between the NTexPE group and the CONTR group. However, insulin in the HT-exPE group was significantly higher than in the NT-exPE group at both 1 and 14 years pp. The concomitantly measured circulating levels of total- and LDL-cholesterol did not differ appreciably between the 2 former patient subgroups. (table 2). However, only in the HT-exPE subgroup, we noticed an increase in circulating LDL levels during the intermeasurement interval with a trend towards significance. In the HT-exPE subgroup we also observed a trend to lower HDL-cholesterol at 14 years pp. $(p=0.06)$, and significantly higher circulating levels of fasting triglycerides at the 14-years pp. measurement. The change in most other variables between 1 and 14 years pp. in the 3 subgroups did not differ appreciably. The rise in systolic BP in the inter-measurement interval was largest in the HT-exPE subgroup and was accompanied by an increase in pulse pressure. 
Table 1: Demography and obstetrical history of the two subgroups of former patients and the parous controls. Data are presented as median with IQR, unless stated otherwise.

\begin{tabular}{|c|c|c|c|}
\hline & $\begin{array}{c}\text { Controls } \\
(n=8)\end{array}$ & $\begin{array}{c}\text { NT-exPE } \\
(n=13)\end{array}$ & $\begin{array}{c}\text { HT-exPE } \\
(n=7)\end{array}$ \\
\hline Age at 1 y pp (years) & $33(32 ; 34)$ & $31(30 ; 33)$ & $30(29 ; 32)$ \\
\hline Age at the time of follow-up (years) & $45(44 ; 47)$ & $43(42 ; 46)$ & $43(41 ; 44)$ \\
\hline Incidence overweight 1 y pp. (n, \%) & $2(25)$ & $3(23)$ & $3(43)$ \\
\hline Incidence overweight 14 y pp. (n, \%) & $2(25)$ & $4(31)$ & $5(71)$ \\
\hline Incidence Pre hypertension (n,\%) & $2(25)$ & $3(23)$ & $4(57)$ \\
\hline Parity at 14 y pp(median, range) & $3(2-4)$ & $2(1-3)$ & $2(2-4)$ \\
\hline Smoking 1 y pp $(n, \%)$ & $1(13)$ & $0(0)$ & $2(29)$ \\
\hline Smoking 14 y pp $(n, \%)$ & $4(50)$ & 0 & 0 \\
\hline \multicolumn{4}{|l|}{ Index pregnancy } \\
\hline Gestational age at birth (wk) & $39.6(38.0 ; 41.7)$ & $34.9(29.6 ; 37.0)^{*}$ & $28.7(27.0 ; 31.1)^{\star \dagger}$ \\
\hline Birthweight (g) & $3360(2800 ; 3645)$ & $2220(1007 ; 2796)^{\star}$ & $920(670 ; 1630)^{\star \dagger}$ \\
\hline $\begin{array}{r}p<5(\text { SGA) } \\
p 5-90 \\
p>90\end{array}$ & $\begin{array}{c}0 / 8 \\
8 / 8(100) \\
0 / 0(0)\end{array}$ & $\begin{array}{c}2 / 13(15) \\
10 / 13(77) \\
1 / 13(8)\end{array}$ & $\begin{array}{c}3 / 7(43) \\
4 / 7(57) \\
0 / 7(0)\end{array}$ \\
\hline Early-onset preeclampsia (n, \%) & - & $6(46 \%)$ & $7(100 \%)^{\dagger}$ \\
\hline \multicolumn{4}{|l|}{ Subsequent pregnancy } \\
\hline Number of subjects (n, \%) & $7(88)$ & $10(77)$ & $7(100)$ \\
\hline Uneventful course/outcome ( $\mathrm{n}, \%)$ & $7(100)$ & $8(80)$ & $1(14)^{\dagger}$ \\
\hline Hypertensive Complication (n, \%) & - & $2(20)$ & $6(86)^{\dagger}$ \\
\hline
\end{tabular}

Overweight: BMl: > $25 \mathrm{~kg} \cdot \mathrm{m}^{2}$; PE: preeclampsia; NT: normotensive; HT: hypertensive; SGA: small for gestational age. ${ }^{*} p<0.05$ compared with controls, $t p<0.05$ compared with NT-exPE. 


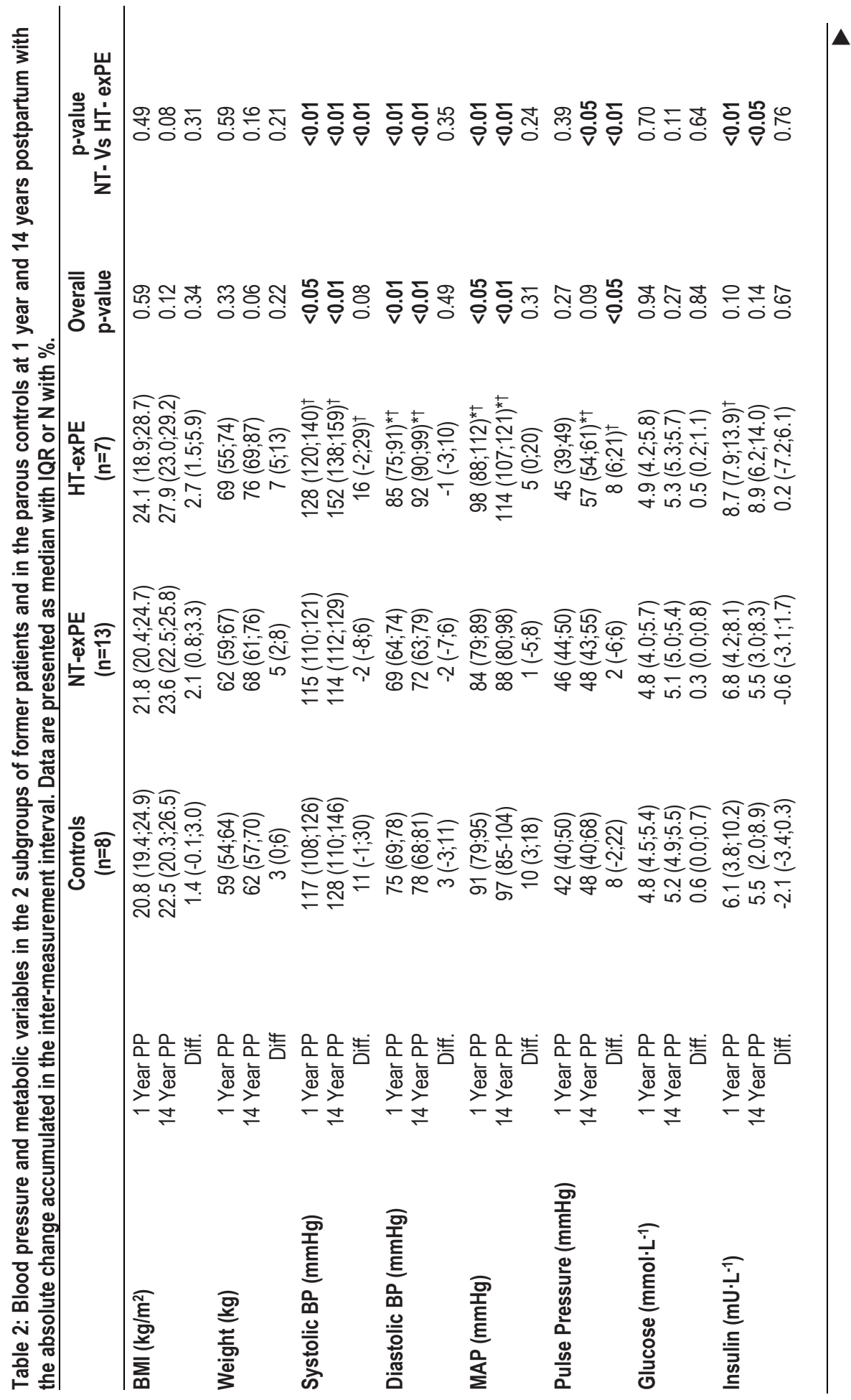




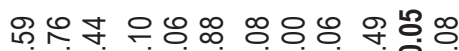

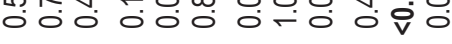

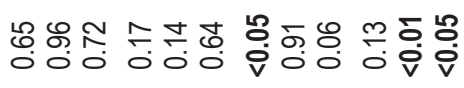

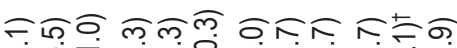

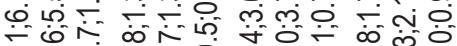

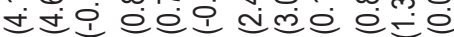
舟

๙

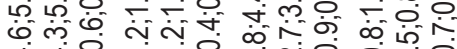

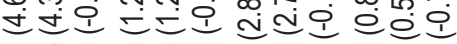

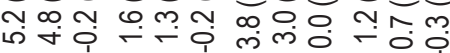

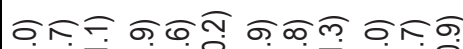

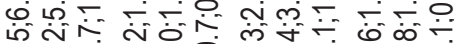

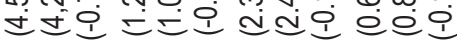
话㧒

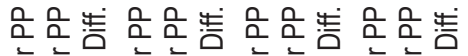

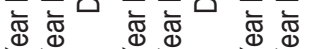

$\searrow \searrow$

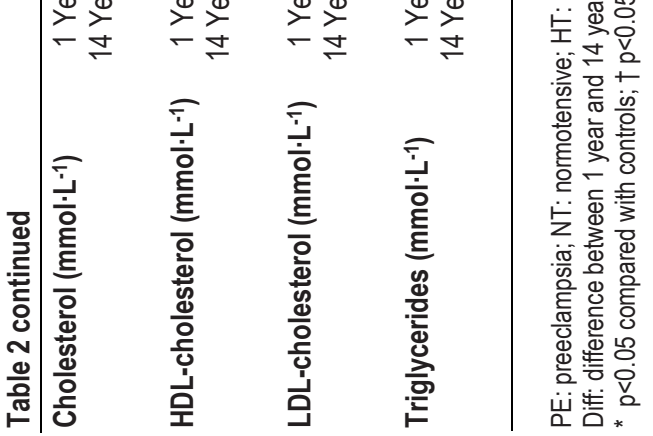




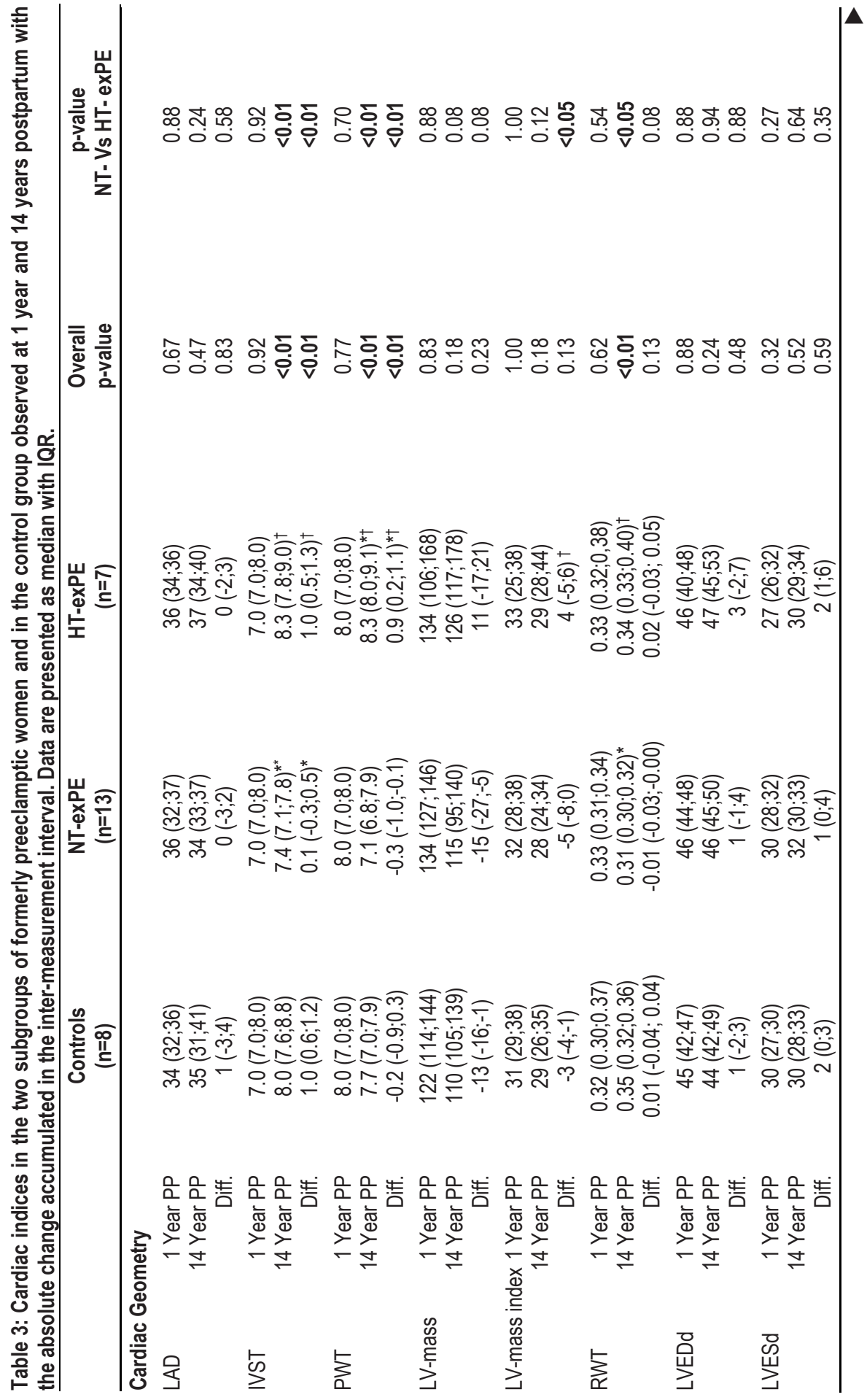




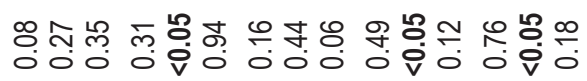

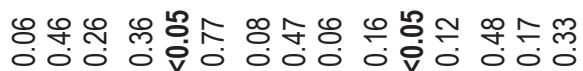

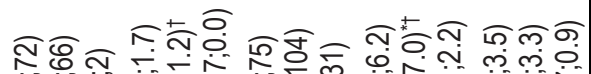
iิ के के के

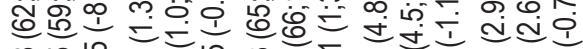
0 每

गु -

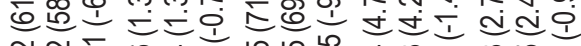

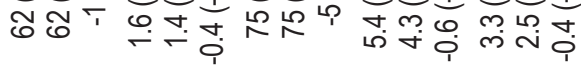

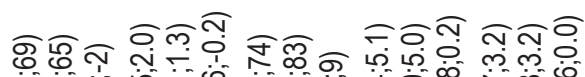

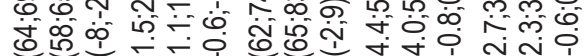
बढ

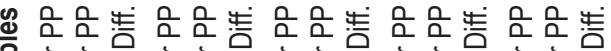

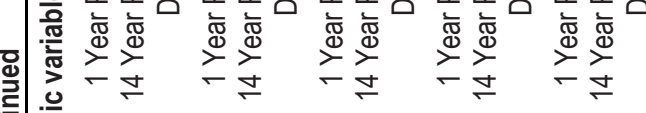
・꼬 닌

$$
\text { 은 }
$$

$$
\text { क }
$$

$$
8
$$$$
\bar{\sigma}
$$ 
Although the cardiac indices in the 3 groups did not differ at 1 year pp. (table 3), HTexPE had deviated from NT-exPE by 14 -years pp. (table 3 ) indicated by a larger IVST, PWT, RWT and a higher CO, and a trend towards a larger LVM relative to NT-exPE. The relative changes in these variables are shown in figure 1. Also the E/A ratio had decreased in the HT-exPE group relative to NT-exPE, without concomitant change in LVEDD and LVESD, suggesting concentric remodeling accompanied by a decline in cardiac diastolic function.

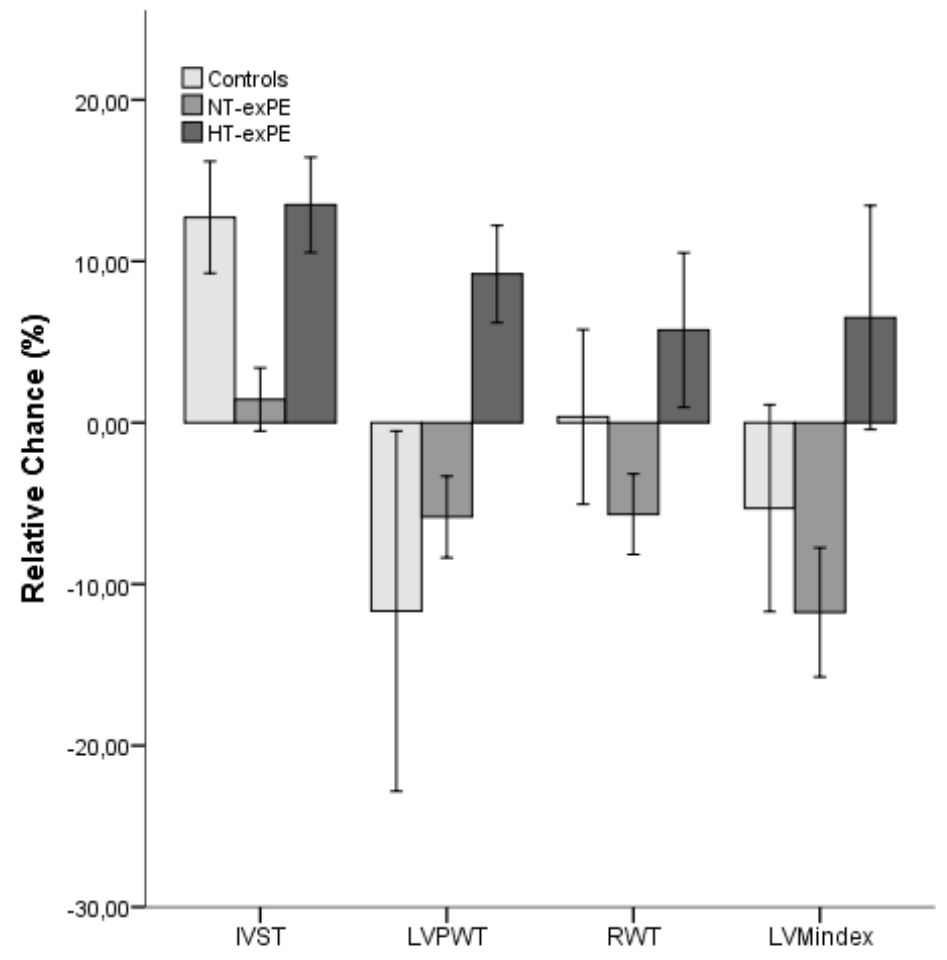

Figure 1: Relative change ( $\pm S E M)$ between 1 year and 14 years postpartum in the control group, normotensive formerly preeclamptics (NT-exPE) and hypertensive formerly preeclamptics (HT-exPE). IVST: Inter-ventricular Septum Thickness; PWT: Posterior Wall Thickness; LVMindex: Left Ventricular Mass index; RWT: Relative Wall Thickness.

${ }^{*} p<0.05$ compared with controls; $\uparrow p<0.05$ compared with NT-exPE.

\section{Discussion}

This longitudinal pilot study in formerly preeclamptic women confirms our hypothesis that former patients destined to develop chronic hypertension, show accelerated left ventricular concentric remodeling relative to their counterparts that remain normotensive. Moreover, this study supports the observation in our earlier crosssectional study ${ }^{6}$ that prehypertension is more prevalent in women destined to develop chronic hypertension. We also noticed in the current study that a history of early-onset 
preeclampsia and recurrence of a hypertensive complication in next pregnancies relates to the development of chronic hypertension. This is in line with previous findings, ${ }^{4}$ but in contrast with a previous study reporting no significant difference in either gestational age of onset or recurrence of hypertensive pregnancy disorder between normotensive and hypertensive former PE women. ${ }^{6}$ A possible explanation for this difference may be differences in demography and study design. In the current study, most women had experienced early-onset PE or PE with concomitant SGA. Early-onset PE appears to be a proxy for underlying cardiovascular dysfunction, thus indicating a higher risk developing chronic hypertension. ${ }^{20}$ Second, in the previous study, we used self-report to determine hypertension in women. This method could have led to underestimation and concomitant bias of the prevalence of hypertension in subjects who do not regularly check their blood pressure. In the current study we used two well-defined moments in which we clinically measured the blood pressure in women and diagnosed chronic hypertension based on standard criteria. Finally, this current study provided indirect evidence for lifestyle-related factors, such as overweight, prehypertension, and raised fasting insulin levels, to add to the risk of developing chronic hypertension. Conversely, in this cohort of former patients, left ventricular mass and RWT at 1-year pp. were not significantly larger in women destined to develop hypertension compared to their counterparts remaining normotensive. These findings are in line with some, ${ }^{21}$ but in contrast to other reports. ${ }^{6,20}$ It is conceivable that the discrepancy with our previously reported crosssectional findings ${ }^{6}$ are related to a difference in the composition of the study populations, whereas also the modest group sizes in this study may have contributed. Prehypertension, defined as systolic and diastolic blood pressures ranging from 120 to 139, and from 80 to $89 \mathrm{mmHg}$, respectively, is an important risk factor for later hypertension and is associated cardiovascular morbidity. ${ }^{22}$ In our study, $57 \%$ of the HTexPE group fulfilled the criteria of pre-hypertension at the 1-year pp. check-up as opposed to only $23 \%$ in NT-exPE. However, this difference did not reach statistical significance, probably due to the modest group sizes. Interestingly, 5 out of 7 former PE patients with prehypertension had developed a recurrent hypertensive disorder in their next pregnancy, suggesting prehypertension to predispose to recurrent $P E$, an finding requiring confirmation in a larger study population.

The higher incidence of overweight in HT-exPE at both measurements sessions, the higher starving insulin levels at 1-year pp., the trend towards a lower HDL-cholesterol and the raised circulating levels of triglycerides at 14 years, may have expedited prehypertension to evolve to chronic hypertension in the HT-exPE subgroup. The prehypertension observed at 1-year pp., may already have been present during the index pregnancy contributing to its adverse outcome and to recurrent PE in next pregnancies.

The mechanism that eventually leads to chronic hypertension in normotensive formerly PE patients is probably multifactorial. A proposed etiology by our group ${ }^{23}$ and others, at least in part supported by the results of this study, refers to the role of the metabolic syndrome (MetS). ${ }^{9,10,24}$ Women with a history of PE frequently exhibit features of MetS, which is in line with our findings. PE and CVD share several common risk factors (such as obesity, insulin resistance and hypertension). It is often suggested that both PE and premature CVD may be manifestations of the MetS. ${ }^{2324,25}$ Therefore, 
it is important to screen former PE patients for underlying additional risk factors to enable the timely institution of preventive measures, thus at least delaying the development of premature CVD.

This study provides unique data on the effect of aging and the superimposed effect of hypertension on cardiac geometry in a relatively young group of women. The observation that $35 \%$ of this young, seemingly healthy population develops chronic hypertension even before the onset of the post-menopause, along with a concentrically remodeling left ventricle is to be considered a serious health problem. This observation may in fact be one of the pathways that relate this former PE population to premature CVD.

The subpopulation of formerly preeclamptic women offers an opportunity to identify women at risk of developing chronic hypertension and premature CVD. This study provides evidence for former patients with prehypertension, a history of early-onset and/or recurrent $\mathrm{PE}$, and/or signs of the metabolic syndrome to be at extra risk to develop chronic hypertension and its unfavorable impact on cardiac structure and function. The obstetrician counseling a former patient after her complicated pregnancy plays a key role in identifying the risk patient, motivating her to adopt relevant lifestyle changes, and referring her for follow-up visits and fine-tuning of preventive measures to a general practitioner, cardiologist or vascular medicine specialist. ${ }^{10}$

Obviously the size of our study population and with it, the statistical power of this study was modest. However, studying a population during a 14-years follow-up period is unique and enabled us to evaluate a wide range of risk factors potentially relevant in the identification of women at risk for later hypertension. This pilot study provides evidence for prehypertension probably being an important "background" risk factor for both PE and chronic hypertension. It is not surprising that external factors such as lifestyle, eating habits, weight gain and their unfavorable metabolic effects magnify the adverse impact of prehypertension, both on pregnancy outcome and later cardiovascular health. These pilot data support the need and the potential of the cardiovascular risk assessment at 1-year $\mathrm{pp}$. in normotensive formerly preeclamptic women, to identify those at risk for later chronic hypertension. 


\section{References}

1. Hernandez-Diaz S, Toh S, Cnattingius S. Risk of pre-eclampsia in first and subsequent pregnancies: prospective cohort study. BMJ 2009;338:b2255.

2. Adams EM, Macgillivray I. Long-term effect of preeclampsia on blood-pressure. Lancet 1961;2:1373-5.

3. Carty DM, Delles C, Dominiczak AF. Preeclampsia and future maternal health. J Hypertens;28:1349-55.

4. Bellamy L, Casas JP, Hingorani AD, Williams DJ. Pre-eclampsia and risk of cardiovascular disease and cancer in later life: systematic review and metaanalysis. BMJ 2007;335:974.

5. Myatt L, Webster RP. Vascular biology of preeclampsia. J Thromb Haemost 2009;7:375-84.

6. Ghossein-Doha C, Peeters L, van Heijster S, et al. Hypertension After Preeclampsia Is Preceded by Changes in Cardiac Structure and Function. Hypertension 2013.

7. Davila DF, Donis JH, Odreman R, Gonzalez M, Landaeta A. Patterns of left ventricular hypertrophy in essential hypertension: should echocardiography guide the pharmacological treatment? International journal of cardiology 2008;124:134-8.

8. Ganau A, Devereux RB, Roman MJ, et al. Patterns of left ventricular hypertrophy and geometric remodeling in essential hypertension. J Am Coll Cardiol 1992;19:1550-8.

9. Barden AE, Beilin LJ, Ritchie J, Walters BN, Michael C. Does a predisposition to the metabolic syndrome sensitize women to develop pre-eclampsia? J Hypertens 1999;17:1307-15.

10. Harskamp RE, Zeeman GG. Preeclampsia: at risk for remote cardiovascular disease. Am J Med Sci 2007;334:291-5.

11. Perry IJ, Beevers DG. The definition of pre-eclampsia. Br J Obstet Gynaecol 1994;101:587-91.

12. Visser GH, Eilers PH, Elferink-Stinkens PM, Merkus HM, Wit JM. New Dutch reference curves for birthweight by gestational age. Early Hum Dev 2009;85:73744.

13. Spaanderman ME, Van Beek E, Ekhart TH, et al. Changes in hemodynamic parameters and volume homeostasis with the menstrual cycle among women with a history of preeclampsia. Am J Obstet Gynecol 2000;182:1127-34.

14. Expert Panel on Detection E, Treatment of High Blood Cholesterol in A. Executive Summary of The Third Report of The National Cholesterol Education Program (NCEP) Expert Panel on Detection, Evaluation, And Treatment of High Blood Cholesterol In Adults (Adult Treatment Panel III). JAMA 2001;285:2486-97.

15. Bonora E, Targher G, Alberiche M, et al. Homeostasis model assessment closely mirrors the glucose clamp technique in the assessment of insulin sensitivity: studies in subjects with various degrees of glucose tolerance and insulin sensitivity. Diabetes Care 2000;23:57-63.

16. DuBois D, DuBois EF. A formula to estimate the approximate surface area if height and weight be known. Arch Int Med 1916;17:863-71. 
17. Devereux RB, Casale PN, Kligfield P, et al. Performance of primary and derived Mmode echocardiographic measurements for detection of left ventricular hypertrophy in necropsied subjects and in patients with systemic hypertension, mitral regurgitation and dilated cardiomyopathy. Am J Cardiol 1986;57:1388-93.

18. de Simone G, Daniels SR, Devereux RB, et al. Left ventricular mass and body size in normotensive children and adults: assessment of allometric relations and impact of overweight. J Am Coll Cardiol 1992;20:1251-60.

19. Allison PD. Missing data techniques for structural equation modeling. J Abnorm Psychol 2003;112:545-57.

20. Melchiorre K, Sutherland GR, Liberati M, Thilaganathan B. Preeclampsia is associated with persistent postpartum cardiovascular impairment. Hypertension 2011;58:709-15.

21. Evans CS, Gooch L, Flotta D, et al. Cardiovascular system during the postpartum state in women with a history of preeclampsia. Hypertension;58:57-62.

22. Chobanian AV, Bakris GL, Black HR, et al. The Seventh Report of the Joint National Committee on Prevention, Detection, Evaluation, and Treatment of High Blood Pressure: the JNC 7 report. Jama 2003;289:2560-72.

23. Spaan JJ, Sep SJ, van Balen VL, Spaanderman ME, Peeters LL. Metabolic syndrome as a risk factor for hypertension after preeclampsia. Obstet Gynecol 2012;120:311-7.

24. Solomon CG, Seely EW. Brief review: hypertension in pregnancy : a manifestation of the insulin resistance syndrome? Hypertension 2001;37:232-9. 


\section{Chapter 6}

\section{Preeclampsia, an independent risk factor for asymptomatic Heart Failure in young women}
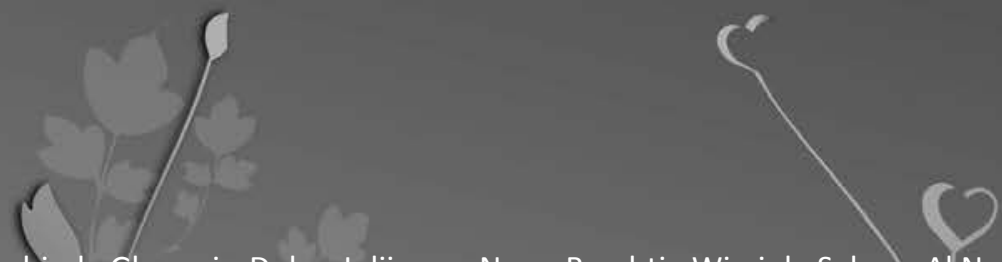

Chahinda Ghossein-Doha; Jolijn van Neer; Brechtje Wissink; Salwan Al Nasiry; Leon J de Windt; Arie PJ van Dijk; Maureen J van der Vlugt, Mirian CH Janssen; Wieteke M

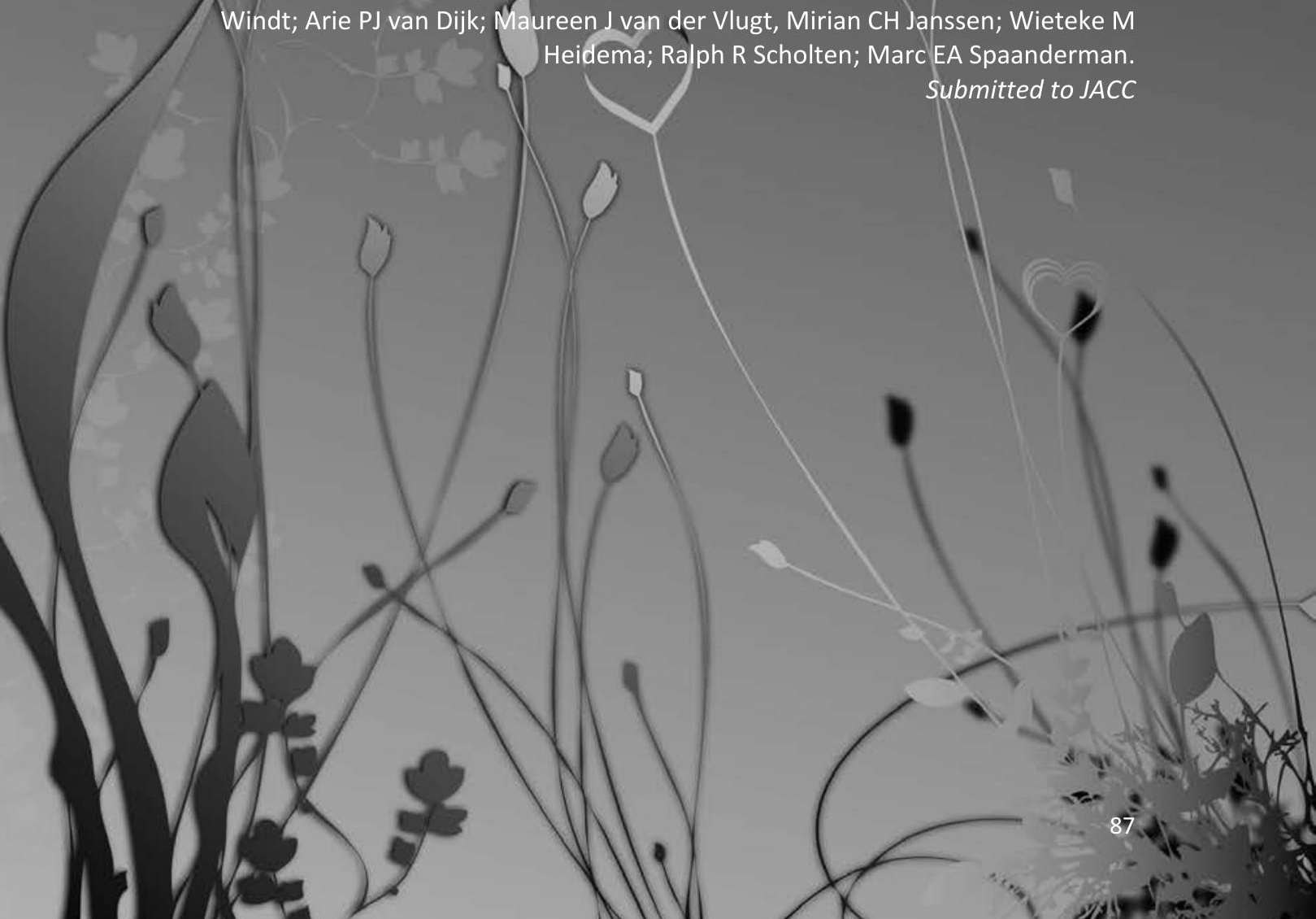




\begin{abstract}
Background: More women than men die of cardiovascular disease in western countries. A history of preeclampsia (PE) is considered a gender-specific risk indicator of cardiovascular disease (CVD) and is associated with a 2-7 fold increased risk for CVD. Asymptomatic heart failure (HF) stage $B$ is thought to precede the progression to mortality related symptomatic stages $C$ and $D$. Whether PE is an independent risk factor for this asymptomatic heart disease is yet unknown.
\end{abstract}

Objective: To test the hypothesis that PE is an independent risk factor for having $\mathrm{HF}$ stage-B at 5-10 years post pregnancy and to explore the contribution of specific risk factors after PE to the development of this asymptomatic stage.

Method: Formerly preeclamptic women $(n=117)$ and women with uneventful pregnancies (controls) $(n=51)$ were invited for cardiovascular risk-management analysis 5-10 years postpartum. Measurements were performed after an overnight fast and included cardiac ultrasound, oscillometric blood pressure (BP) measurement and metabolic syndrome determinants. HF stage-B was diagnosed according to the ASE guidelines. Univariate and multivariate regression analysis were performed to calculate (adjusted) odd's ratios (OR) for variables associated with HF stage-B.

Results: The prevalence of HF stage-B was $\sim 4$ fold higher in the formerly preeclamptic group compared to controls ( $21 \%$ vs. $6 \%, \mathrm{p}<0.01)$. Most women $(76 \%)$ with HF stage-B had preserved ejection fraction (EF). When adjusted for cardiovascular risk factors that where significant in the univariate analysis (BMI, fasting insulin and BP) and postpartum interval, $\mathrm{PE}$ remained independently significantly associated with HF stageB (aOR: 11.9 (95\% Cl 2.1 - 66.7).

Conclusions: PE increases the risk eleven fold for having HF stage-B at five to ten years postpartum and most of these women have HF with preserved EF. Moreover, unfavorable, but modifiable metabolic syndrome constituents relate to this condition. 


\section{Introduction}

From a worldwide perspective, cardiovascular disease (CVD) is the leading cause of disability and death. ${ }^{1-3}$ In western countries, more women than men die of CVD ${ }^{4,5}$, making CVD in women an important public health issue. In addition to the genderindependent classical risk factors seen in both men and women, the woman specific risk factor preeclampsia (PE) increases the risk for CVD 2 to 7 fold. ${ }^{6-10}$

$\mathrm{PE}$ is considered a maternal vascular disorder and is defined as new onset hypertension after 20 weeks of gestation along with proteinuria. ${ }^{11}$ It complicates 5$10 \%$ of first pregnancies. ${ }^{8,12}$ In contrast to the physiological eccentric remodeling during healthy pregnancies, a preeclamptic pregnancy is characterized by the development of concentric left ventricular remodeling. ${ }^{13}$ The additional increase in left ventricular mass (LVM) in women with PE compared to healthy pregnancies ${ }^{14,15}$ may not always resolve postpartum. ${ }^{16} \mathrm{PE}$ related residual increased left ventricular mass may relate to an increased prevalence of Heart Failure (HF) stage-B up to one year postpartum in this relatively young female population. ${ }^{16}$

HF stage-A (presence of risk factors) and stage-B (functional alterations) are considered to be a continuum in asymptomatic preclinical stages that precede the development towards the clinical HF stages-C and -D (structural heart disease in combination with symptomatic disease). ${ }^{17}$ The progression from the preclinical stage $B$ to the clinical stage $\mathrm{C}$ is associated with a 5-fold increase in cardiovascular related mortality ${ }^{18,19}$, decreased quality adjusted life years and with it, higher healthcare costs. ${ }^{19}$ Early detection and tailored intervention of women with HF stage-B decreases progression to stage $\mathrm{C}$, and might therefore improve clinical outcome and cardiovascular related mortality. ${ }^{18,19}$

It is not known whether the reported high prevalence of HF stage-B one year postpartum in formerly preeclamptic women still is a slowly resolving process that may diminish in a forthcoming period, or whether this high prevalence persists or even progresses in the subsequent years. If the latter is true, this young female population at risk should be offered cardiovascular risk management to prevent early onset heart failure. In this study, we tested the hypothesis that the prevalence of HF stage-B is elevated in formerly PE women compared to healthy controls 5-10 years postpartum and that PE is an independent risk factor for this asymptomatic stage. Besides, we explored the additional role of more traditional risk factors associated with HF stage-B. To this end, we conducted a case-control study in which we quantified the presence of cardiovascular risk factors and assessed cardiac functioning.

\section{Methods}

\section{Study design}

In this case control study, formerly preeclamptic women and women with uneventful pregnancies (healthy parous controls) were invited for cardiovascular risk management analysis 5-10 years postpartum. Formerly preeclamptic women that participated in a previous study $(n=121)$ were invited to participate in this study by mail. Of these women that were invited, four women did not have any cardiovascular measurement 
performed. And where excluded from analysis resulting. Consequently, 117 former PE patients were eligible for analysis. Healthy parous controls were recruited by advertisement. Fifty-seven women contacted us initially for participation. Of these women, six women did not show up and where excluded. Finally, 51 women where included in the control group for analysis. As the system biology towards preeclampsia is considered heterogeneous, we included at least two formerly PE women for each healthy parous control.

The Nijmegen Medical Centre Medical Ethics Committee approved our study protocol before patient enrolment (NL32718.091.10). The procedures followed were in accordance to institutional guidelines. Informed consent was given before patient enrolment and procedures adhered to the principles of the Declaration of Helsinki and Title 45, U.S. Code of Federal Regulations, Part 46, Protection of Human Subjects, Revised November 13, 2001, effective December 13, 2001.

\section{Study population}

Non-pregnant women were eligible for inclusion if they had experienced PE during their pregnancy. All women included were Caucasian. Medical history was taken, including family history regarding cardiovascular disease (< age 60 year) in first line relatives. Besides, obstetric data regarding offspring was taken including intra uterine fetal demise (IUFD), birth weight and birth weight centile (according to the national birth weight charts).(20) Infants born were considered small for gestational age (SGA) when birth weight corrected for gestational age $<5^{\text {th }}$ centile. PE was diagnosed using the criteria as defined by the International Society for the Study of Hypertension in Pregnancy. ${ }^{21}$ PE was defined as new-onset hypertension (systolic blood pressure (SBP) $\geq 140 \mathrm{mmHg}$ or diastolic blood pressure (DBP) $\geq 90 \mathrm{mmHg}$ ) after 20 weeks of gestation and concomitant proteinuria exceeding $0.3 \mathrm{~g} /$ day. Early onset PE was defined as PE developing before 34 gestational weeks and leading to birth $<34$ weeks, preterm PE was defined as birth occurring before 37 weeks of gestation. Women were eligible to be included in the control group if they had a history of uncomplicated pregnancy, their pregnancy charts were checked to ensure an uneventful pregnancy. In both groups, we excluded women who had, prior to their first pregnancy, chronic hypertension, diabetes or autoimmune disease.

\section{Measurements}

Cardiovascular risk factor screening and echocardiographic measurements were performed after an overnight fast. Screening for cardiovascular risk consisted of automated blood pressure measurement, body weight $(\mathrm{kg})$ and length $(\mathrm{m})$, and laboratory measurements to detect metabolic syndrome (MetS). All measurements started at 8:00 am in a temperature-controlled room $\left(20^{\circ} \mathrm{C}\right)$. After 30 minutes rest in sitting position, blood pressure $(\mathrm{BP}, \mathrm{mmHg})$ and heart rate $(\mathrm{HR}, \mathrm{bpm})$ were measured oscillometrically (Dinamap Vital Signs Monitor 1846; Critikon, Tampsa, Florida USA) with the cuff size recommended for the arm circumference, at a 3-minute intervals for 30 minutes at the right upper arm. We recorded systolic (SBP) and diastolic (DBP) blood pressures, mean arterial pressure (MAP) and heart rate (HR) from which we used the median values for analysis. Hypertension was diagnosed as SBP $\geq 140 \mathrm{mmHg}$ 
and/or DBP $\geq 90 \mathrm{mmHg}$ or the use of antihypertensive medication. Prehypertension was diagnosed as SBP $\geq 120$ - $139 \mathrm{mmHg}$ and/or DBP $\geq 80-89 \mathrm{mmHg}$.

Height and body mass (Seca 888 scale, Hamburg, Germany) were measured. Body mass index (BMI) was calculated by dividing body weight $(\mathrm{kg})$ by the squared height $\left(m^{2}\right)$.

All participants collected urine in the 24 hours preceding the measurements. The 24 hours urine sample was assayed for albumin and creatinine to define the (micro) albuminuria corrected for creatinine output $(\mathrm{g} / \mathrm{mol}$ creatinine) (Aeroset, Abbot Laboratories, Illinois USA). Venous blood samples were taken from the antecubital vein and analyzed for metabolic parameters: glucose, insulin, high density lipoprotein and triglycerides (Aeroset, Abbot Laboratories, Illinois USA). The product of fasting glucose and insulin (homeostasis model assessment index for insulin resistance, $\mathrm{HOMA}_{\mathbb{R}}$ ) was calculated (insulin $[\mathrm{mU} / \mathrm{L}] \times$ glucose $[\mathrm{mmol} / \mathrm{L}] / 22.5$ ) to estimate insulin resistance.

The diagnosis of MetS and the cut-off values of its constituents were based on the criteria set by the World Health Organization (WHO). We defined MetS by the concomitant presence of insulin resistance (fasting insulin $\geq 9.2 \mathrm{mU} / \mathrm{L}$ or fasting glucose $\geq 6.1 \mathrm{mmol} / \mathrm{L}$ or $\mathrm{HOMA}_{\mathrm{R}} \geq 2.2$ ) and two or more of the following factors: hypertension (systolic blood pressure $\geq 140 \mathrm{mmHg}$ or diastolic blood pressure $\geq 85 \mathrm{mmHg}$ or the use of anti-hypertensive medication), obesity (BMI $\geq 30 \mathrm{~kg} / \mathrm{m}^{2}$ ), dyslipidemia (triglycerides $\geq 1.69 \mathrm{mmol} / \mathrm{l}$ or $\mathrm{HDL} \leq 0.9 \mathrm{mmol} / \mathrm{l}$ ) and/or microalbuminuria (urine albumin to creatinine ratio $\geq 2.5 \mathrm{~g} / \mathrm{mol}$ creatinine).

\section{Cardiographic measurements}

Echocardiographic measurements were obtained using a phased-array echocardiographic Doppler system (Vivid 7, General Electric, Horten, Norway). We performed the two-dimensional, M-mode and Doppler echocardiography according to the guidelines of the American Society of Echocardiography (ASE). ${ }^{22}$ Using M-mode in the parasternal long-axis view, we measured left ventricular end-diastolic and endsystolic diameters (LVEDd, LVESd, $\mathrm{mm}$ ), as well as end-diastolic thickness of the interventricular septum (IVST, $\mathrm{mm}$ ) and of the posterior wall (PWT, mm). Left ventricular mass (LVM, g) was calculated as recommended by the ASE using the formula $0.8 \times\left(1.04\left((\text { LVIDd+PWTd }+ \text { SWTd })^{3}-(\text { LVIDd })^{3}\right)\right)+0.6$ and indexed for BSA. ${ }^{23}$ The relative wall thickness (RWT) was calculated using the formula

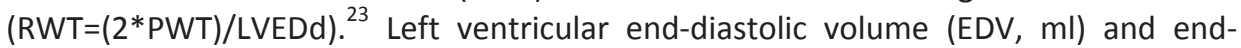
systolic volumes (ESV, ml) were estimated using the Teichholz-formula. ${ }^{24}$ Ejection fraction was calculated by $E F(\%)=((E D V-E S V) /(E D V)) \cdot 100$.

The heart rate (HR, beats/min) was obtained by taking the reciprocal of the mean of 5 consecutive RR-intervals on the electrocardiogram (ECG) multiplied by 60 . We estimated the mean aortic Velocity Time Integral (VTI) by averaging the outer edge tracings of 5 consecutive Continuous Wave (CW) Doppler registrations of the left ventricular outflow tract velocity. By taking the product of VTI and the mid-systolic cross-sectional area at the level of the left ventricular outflow tract in the parasternal long axis view, we obtained stroke volume (SV, $\mathrm{mL}$ ). Cardiac output (CO, L/min) was obtained by multiplying SV with HR. The assessments were performed off-line using EchoPAC PC SW, by Vingmed Ultrasound, Version 6.1.2. 


\section{Outcome measures}

The subclinical stages of HF were diagnosed according to the American Heart Association AHA.(17) Stage A was defined as the presence of at least one risk factor for heart failure, including hypertension, atherosclerotic disease, diabetes, obesity, MetS, use of cardio toxins or a family history of cardiomyopathy. ${ }^{17} \mathrm{HF}$ stage-B was defined as the presence of previous myocardial infarction, left ventricular concentric remodeling or hypertrophy, loss of systolic function, or asymptomatic valvular disease. ${ }^{23}$ Left ventricular remodeling was defined as a left ventricular mass index (LVMi) $>95 \mathrm{~g} / \mathrm{m}^{2}$, concentric remodeling (RWT $>0.42$ and $L V M i<95 \mathrm{~g} / \mathrm{m} 2$ ) or ejection fraction $<55 \%$, according to the ASE guidelines. ${ }^{23}$ We defined asymptomatic valve disease as mild aorta valve insufficiency, mild thickening of mitral valve or central aorta valve insufficiency. To define low or preserved EF in this subclinical stage, we used the cutoff value of mildly abnormal based on the ASE guidelines. ${ }^{23} \mathrm{HF}$ stage-B with preserved ejection fraction (HFpEF) was defined as HF stage-B with $E F \geq 55 \%$.

Patients where defined as not classified when they did not have a complete cardiac ultrasound, set and none of the known criteria was positive. When at least one of the criteria for HF stage-B was present in the incomplete dataset, they were allocated to the group of HF stage-B.

\section{Data analysis (statistical test and handling data)}

Data were analysed using SPSS version 21. If data required for the heart failure classification were missing, women were categorized as 'not classified'. To analyze differences between women with a history of PE and controls, we used chi-square for categorical data if $\geq 5$ cases were present and the Fisher exact test if 1-5 cases were present. We used the independent T-test for normally distributed continuous variables en detailed data as mean with SD. A two sided p-value of $<0.05$ was considered statistically significant.

To study the association of several variables with HF stage-B in the whole population, we used univariate regression analysis to calculate the odds ratios (OR). An event was defined as HF stage-B vs no HF at all. Factors that were significantly associated in the univariate analysis $(p<0.05)$ were used in the multivariate logistic regression analysis to adjust the odds ratio for PE to HF stage-B. Next, within the group of former PE patients, variables associated with HF stage- $B$ were analysed with the univariate regression analysis. An event was defined as HF stage-B vs no HF stage-B.

We compared the unclassified women in each group with the classified women to test for possible bias related to baseline characteristics.

A sample size analysis was performed based on previous findings by Melchiorre et al. on the prevalence of HF stage-B at one year postpartum in former PE patients (43\%) compared to controls (10\%).(16) The required sample size was calculated with a desired power of 0.90 and a two sided $\alpha$ of 0.05 . To this end a sample size of at least 36 people for each group was necessary to detect a significant difference. 


\section{Results}

In total, 168 women were included in our study, of which 117 women with a history of PE and 51 controls (figure 1). Of the former PE patients, 11 (9\%) women did not have a complete measurements set and could not be classified. In the control group, $10(20 \%)$ women could not be classified due to an incomplete measurement set. Baseline characteristics of the PE and control group are detailed in Table 1. The control group was slightly older and had their postpartum measurement on average 3 years later than the formerly PE group. Furthermore, BMI, fasting insulin and SBP and DBP were higher and HDL lower in formerly preeclamptic women compared to controls. Moreover, MetS and hypertension were more often present in the PE group compared to the control group.

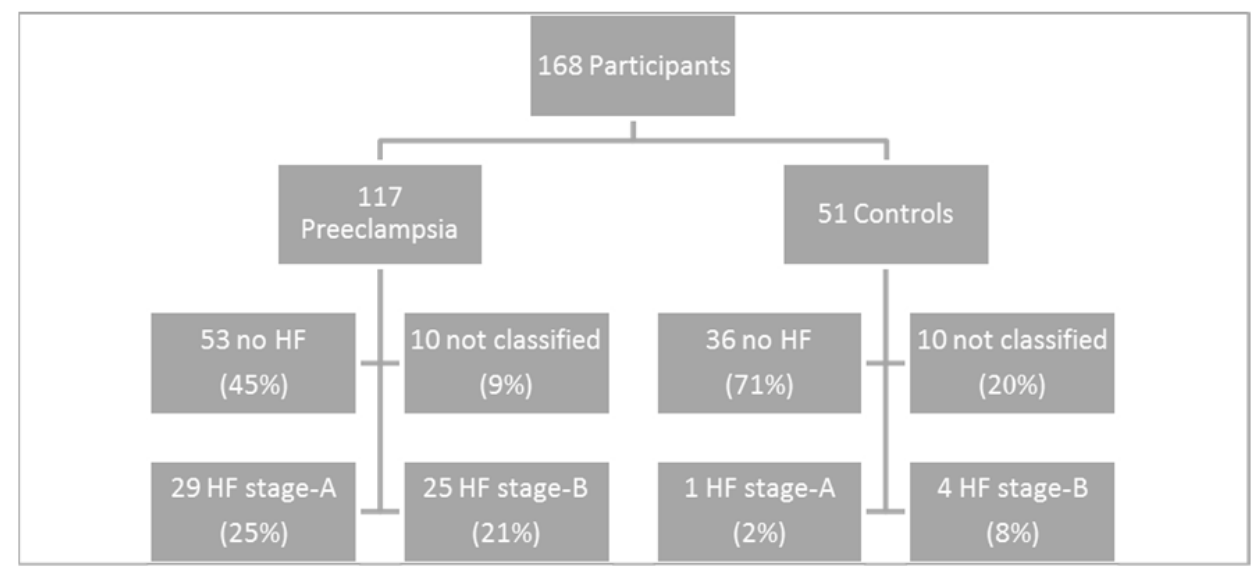

Figure 1: Flowchart of participants and heart failure classification ; HF: Heart Failure

As shown in Table 2 and Fig. 1, the former PE group had a 6 fold (46\% versus 8\%, $\mathrm{p}<0.05)$ higher prevalence of HF. HF stage-A and stage-B were both more prevalent in the PE group compared to the control group: $24 \%$ versus $2 \%(p<0.01)$ and $21 \%$ versus $6 \%(p<0.01)$, respectively. $19 / 25$ (76\%) formerly preeclamptic women with HF stage-B had HFpEF. Concentric remodeling was also more often present in the former PE group $16 / 25(64 \%)$ than in the control group 1/33 (33\%). Three women from the former PE group had valve disease compared to non from the control group while none of both groups had a previous myocardial infarction. However, RWT, LVMi, IVST, PWT and LVEDD and LVEDV, SV, CO and EF were all not different between both groups. 
Table 1: Baseline characteristics 5 to 10 years postpartum and obstetric history of formerly preeclamptic women and controls. Data is presented as mean with SD or median with IQR unlesS otherwise specified.

\begin{tabular}{|c|c|c|c|}
\hline & $\begin{array}{c}\begin{array}{c}\text { Formerly PE } \\
\mathrm{N}=117\end{array}\end{array}$ & $\begin{array}{c}\text { Controls } \\
\mathrm{N}=51\end{array}$ & $p$-value \\
\hline \multicolumn{4}{|l|}{ Patient characteristics } \\
\hline Age, years & $36 \pm 4$ & $39 \pm 4$ & $<0.001$ \\
\hline Weight, kg & $74 \pm 18$ & $69 \pm 12$ & $<0.05$ \\
\hline $\mathrm{BMI}, \mathrm{kg} / \mathrm{m}^{2}$ & $26 \pm 6$ & $23 \pm 3$ & $<0.001$ \\
\hline Smoking, n (\%) & $9(8 \%)$ & $6(12 \%)$ & 0.40 \\
\hline Family history of CVD, $\mathrm{n}(\%)$ & $50(43 \%)$ & $22(43 \%)$ & 0.92 \\
\hline \multicolumn{4}{|l|}{ Obstetric variables } \\
\hline Years postpartum & $5 \pm 3$ & $8 \pm 3$ & $<0.001$ \\
\hline GA at birth, weeks & $33 \pm 4$ & $40 \pm 2$ & $<0.001$ \\
\hline Birth weight, g & $1783 \pm 942$ & $3367 \pm 574$ & $<0.001$ \\
\hline SGA (<P5), n (\%) & $29(25)$ & $3(6)$ & $<0.05$ \\
\hline IUFD, n (\%) & $9(8)$ & $0(0)$ & N.A. \\
\hline \multicolumn{4}{|l|}{ Metabolic syndrome } \\
\hline Metabolic syndrome, n (\%) & $12(10 \%)$ & $0(0 \%)$ & N.A. \\
\hline Fasting insulin (mU/L) & $8.0(5.6-10.3)$ & $5.8(4.2-7.7)$ & $<0.001$ \\
\hline Fasting glucose (mmol/l) & $4.8(4.5-5.0)$ & $4.6(4.4-5.0)$ & 0.36 \\
\hline $\mathrm{HOMA}_{\mathbb{I R}}$ & $1.7(1.2-2.4)$ & $1.2(0.8-1.7)$ & $<0.001$ \\
\hline $\mathrm{HDL}$ (mmol/L) & $1.3 \pm 0.3$ & $1.6 \pm 0.3$ & $<0.001$ \\
\hline Triglycerides (mmol/L) & $1.0 \pm 0.5$ & $0.9 \pm 0.4$ & 0.12 \\
\hline Albumin Creatinine ratio ( $\mathrm{g} / \mathrm{mol})$ & $0.7(0.3-1.4)$ & $0.5(0.4-0.8)$ & 0.60 \\
\hline \multicolumn{4}{|l|}{ Blood pressure } \\
\hline $\mathrm{SBP}, \mathrm{mmHg}$ & $117 \pm 13$ & $110 \pm 10$ & $<0.001$ \\
\hline $\mathrm{DBP}, \mathrm{mmHg}$ & $74 \pm 10$ & $71 \pm 8$ & $<0.05$ \\
\hline $\mathrm{HR}(\mathrm{bpm})$ & $67 \pm 11$ & $61 \pm 10$ & $<0.001$ \\
\hline Prehypertension, n (\%) & $23(23 \%)$ & $10(20 \%)$ & 0.46 \\
\hline Hypertension, n (\% & $27(23 \%)$ & $1(2 \%)$ & $<0.001$ \\
\hline
\end{tabular}

PE: preeclampsia; GA, gestational age; SGA: small for gestational age; IUFD: intrauterine fetal death; HOMAIR: homeostatic model assessment BMI: body mass index; CVD: cardiovascular disease; HDL: high-density lipoprotein; SBP: systolic blood pressure; DPB: diastolic blood pressure; HR: heart rate; 
Table 2: Prevalence of HF stage-A and -B and cardiac function in formerly preeclamptic women and controls 5 to 10 years postpartum.

\begin{tabular}{|c|c|c|c|}
\hline & $\begin{array}{c}\text { Formerly PE } \\
\mathrm{N}=117\end{array}$ & $\begin{array}{c}\begin{array}{c}\text { Controls } \\
\mathrm{N}=51\end{array}\end{array}$ & $\mathrm{p}$-value \\
\hline Subclinical Heart failure & $54(46 \%)$ & $4(8 \%)$ & $<0.05$ \\
\hline $\begin{array}{l}\text { HF Stage-A, n (\%) } \\
\text { HF Stage-B, n (\%) }\end{array}$ & $\begin{array}{l}29(24 \%) \\
25(21 \%)\end{array}$ & $\begin{array}{l}1(2 \%) \\
3(6 \%)\end{array}$ & $\begin{array}{l}<0.01 \\
<0.01\end{array}$ \\
\hline Impaired EF, n (\%) & $6(5 \%)$ & $2(4 \%)$ & 1.00 \\
\hline Concentric remodeling, $\mathrm{n}(\%)$ & $16(14 \%)$ & $1(2 \%)$ & $<0.05$ \\
\hline LVMi > 95 g/m², n (\%) & $1(1 \%)$ & $0(0 \%)$ & NA \\
\hline Previous MI, n (\%) & $0(0 \%)$ & $0(0 \%)$ & NA \\
\hline Valve disease, $\mathrm{n}(\%)$ & $3(3 \% \mid)$ & $0(0 \%)$ & NA \\
\hline HFpEF, n (\%) & $19(16 \%)$ & $1(2 \%)$ & $<0.05$ \\
\hline \multicolumn{4}{|l|}{ Cardiac geometry and function } \\
\hline LVMi, $g / \mathrm{m}^{2}$ & $60 \pm 13$ & $62 \pm 12$ & 0.23 \\
\hline IVST (cm) & $0.76 \pm 0.16$ & $0.74 \pm 0.09$ & 0.70 \\
\hline PWT (cm) & $0.77 \pm 0.14$ & $0.76 \pm 0.11$ & 0.57 \\
\hline LVEDD (cm) & $4.5 \pm 0.4$ & $4.6 \pm 0.4$ & 0.13 \\
\hline RWT & $0.35 \pm 0.07$ & $0.33 \pm 0.05$ & 0.19 \\
\hline LVEDV (mL) & $92 \pm 22$ & $89 \pm 22$ & 0.34 \\
\hline $\mathrm{CO}(1 / \mathrm{min})$ & $5.2 \pm 1.1$ & $4.9 \pm 1.2$ & 0.18 \\
\hline $\mathrm{SV}(\mathrm{mL})$ & $80 \pm 18$ & $75 \pm 17$ & 0.09 \\
\hline $\mathrm{EF}(\%)$ & $63 \pm 6$ & $63 \pm 6$ & 0.61 \\
\hline
\end{tabular}

HF: heart failure; PE: preeclampsia; HFpEF: heart failure with preserved ejection fraction; HFrEF: heart failure with reduced ejection fraction; MI: myocard infarct; LVMi: left ventricular mass index; IVST: interventricular septum thickness; PWT: posterior wall thickness; LVEDD: left ventricular end-diastolic diameter; RWT: relative wall thickness; SV: stroke volume; CO: cardiac output; EF: ejection fraction;

Table 3 presents the obstetric, cardiometabolic and cardiovascular risk factors in relation to HF stage-B. An event was defined as HF stage-B vs no HF at all in the studied population of formerly preeclamptic women en the control group. On the one hand, $\mathrm{PE}$ in general was associated with the HF stage-B with an OR of 5.8 (1.6-20.7) as did also early onset and preterm PE. Moreover, increased BMI, elevated fasting insulin levels, increased systolic and diastolic blood pressure were also associated with HF stage-B. On the other hand, glucose, lipid spectrum and heart rate were not associated with HF stage-B.

After adjusting for the factors that were significant in the univariate analysis (BMI, insulin, SBP, DBP and postpartum interval) PE remained independently, significantly associated with HF stage-B (adjusted OR 11.9; 95\% Cl 2.1-66.7).

Table 4 presents the variables that are, within the PE group, associated with HF stageB. An event for this analysis was defined as HF stage-B vs no HF stage-B. Age, smoking and a family history of CVD were not associated with HF stage-B within the group of formerly PE women. Moreover, al the constituents of MetS were not significantly associated with HF stage-B within the former PE group. However, prehypertension was more often present in the group of former PE women with HF stage-B than in the 
subgroup without HF stage-B (36\% versus $16 \% ; p<0.01)$ with an odd's ratio of 3.9 $(95 \% \mathrm{Cl} 1.3-11.9 ; \mathrm{p}<0.01)$. A history of early $\mathrm{PE}$, preterm PE or recurrent $\mathrm{PE}$ were not significantly different between both subgroups.

Comparison of the unclassified with the classified women in the control group revealed that there were no differences in baseline characteristics beside a slightly older classified group compared to the unclassified group ( $40 \pm 4$ vs $37 \pm 3$ years, $p<0.05$ ). In the former PE group both subgroups did not differ from each other in demography and obstetric history.

Table 3: Odds Ratios of obstetric history, cardiovascular and cardiometabolic factors for HF stage-B vs no HF at all in the whole study population

\begin{tabular}{|c|c|c|}
\hline & OR HF Stage-B $(95 \% \mathrm{Cl})$ & $p$-value \\
\hline \multicolumn{3}{|l|}{ Obstetric variables } \\
\hline Preeclampsia & $5.8(1.6-20.7)$ & $<0.01$ \\
\hline Early onset preeclampsia & $7.2(1.9-27.1 .0)$ & $<0.01$ \\
\hline Preterm preeclampsia & $6.0(1.6-22.0)$ & $<0.01$ \\
\hline Postpartum interval & $1.1(0.9-1.2)$ & 0.36 \\
\hline \multicolumn{3}{|l|}{ Patient characteristics } \\
\hline Age & $0.9(0.9-1.0)$ & 0.25 \\
\hline $\mathrm{BMI}$ & $1.3(1.1-1.4)$ & $<0.001$ \\
\hline Smoking & $1.4(0.3-5.9)$ & 0.63 \\
\hline Family history of CVD & $0.7(0.3-1.7)$ & 0.39 \\
\hline \multicolumn{3}{|l|}{ Metabolic syndrome } \\
\hline Glucose (mmol/l) & $2.3(0.8-6.5)$ & 0.13 \\
\hline Fasting insulin, (mU/l) & $1.2(1.1-1.4)$ & $<0.01$ \\
\hline HOMAIR & $2.3(1.3-4.0)$ & $<0.01$ \\
\hline $\mathrm{HDL}(\mathrm{mmol} / \mathrm{l})$ & $0.2(0.0-1.1)$ & 0.06 \\
\hline Triglycerides (mmol/l) & $2.1(0.7-5.9)$ & 0.18 \\
\hline Albumin Creatinine ratio & $1.0(0.8-1.3)$ & 0.87 \\
\hline \multicolumn{3}{|l|}{ Blood Pressure } \\
\hline $\mathrm{SBP}(\mathrm{mmHg})$ & $1.1(1.0-1.2)$ & $<0.001$ \\
\hline $\mathrm{DBP}(\mathrm{mmHg})$ & $1.1(1.0-1.2)$ & $<0.01$ \\
\hline HR (beats.. min- ${ }^{1}$ ) & $1.0(1.0-1.1)$ & 0.08 \\
\hline Prehypertension & $7.0(2.4-19.9)$ & $<0.001$ \\
\hline Multivariate analyses & $\mathrm{aOR}(95 \% \mathrm{Cl})$ & $\mathrm{p}$-value \\
\hline Preeclampsia & $11.9(2.1-66.7)$ & $<0.01$ \\
\hline
\end{tabular}

PE: preeclampsia; GA: gestational age; SGA: small for gestational age; IUFD: intrauterine fetal death; BMl: body mass index; HDL: high-density lipoprotein; SBP: systolic blood pressure; DPB: diastolic blood pressure. Multivariate analysis is adjusted for BMI, Insulin, SBP and DBP and postpartum interval in years. 
Table 4: Univariate analysis of obstetric history, metabolic syndrome constituents in formerly PE patients who had HF stage-B vs no HF stage-B.

\begin{tabular}{|c|c|c|c|}
\hline & $\begin{array}{c}\text { HF Stage-B } \\
\quad \mathrm{N}=25\end{array}$ & $\begin{array}{c}\text { No HF stage-B } \\
N=82\end{array}$ & OR (CI 95\%) \\
\hline \multicolumn{4}{|l|}{ Patient characteristics } \\
\hline Age (y) & $35 \pm 5$ & $36 \pm 4$ & $1.0(0.9-1.1)$ \\
\hline Smoking, n (\%) & $2(8 \%)$ & $5(6 \%)$ & $1.3(0.2-7.4)$ \\
\hline Family history CVD, n (\%) & $8(32 \%)$ & $38(46 \%)$ & $0.6(0.2-1.5)$ \\
\hline \multicolumn{4}{|l|}{ Obstetric variables } \\
\hline Early onset PE, n (\%) & $17(68 \%)$ & $54(55 \%)$ & $1.1(0.4-2.9)$ \\
\hline Recurrent PE, n (\%) & $8(32 \%)$ & $16(20 \%)$ & $2.1(0.7-6.3)$ \\
\hline Preterm PE, n (\%) & $19(76 \%)$ & $65(79 \%)$ & $0.8(0.3-2.4)$ \\
\hline Metabolic syndrome, $\mathrm{n}(\%)$ & $3(12 \%)$ & $6(7 \%)$ & $1.8(0.4-7.8)$ \\
\hline Insulin resistance, n (\%) & $9(43 \%)$ & $33(40 \%)$ & $0.9(0.3-2.2)$ \\
\hline Obesity, n (\%) & $7(28 \%)$ & $11(13 \%)$ & $2.7(0.9-7.9)$ \\
\hline Dyslipidemia, n (\%) & $0(0 \%)$ & $5(6 \%)$ & NA \\
\hline Microalbuminuria, n (\%) & $4(16 \%)$ & $12(15 \%)$ & $1.2(0.3-4.1)$ \\
\hline \multicolumn{4}{|l|}{ Blood pressure } \\
\hline Hypertension WHO, n (\%) & $5(20 \%)$ & $21(26 \%)$ & $0.8(0.3-2.3)$ \\
\hline Prehypertension, n (\%) & $9(36 \%)$ & $13(16 \%)^{\star}$ & $3.9(1.3-11.9)^{\star}$ \\
\hline
\end{tabular}

PE: preeclampsia; CVD: Cardiovascular Disease; WHO: World Health Organization.

${ }^{*} \mathrm{P}<0.05$

\section{Discussion}

In this Case Control study, we found that the prevalence of the pre-clinical HF stage-B (mainly HFpEF) is $\sim 4$ times higher in women with a history of preeclampsia (21\%) compared to controls (6\%). Even though heart failure is related to MetS, correction for these factors suggested that PE increases independently more than 10 times the risk for HF stage-B. Our findings are in line with previous findings related to the prevalence of HF stage-B up to one year postpartum in formerly preeclamptic women.(16) Moreover, with our study we demonstrate that the high prevalence of HF stage-B even persists in remote postpartum years.

Several components of the MetS related to HF stage-B. In our study an increase in fasting insulin levels and $\mathrm{BMI}$ and a decrease in $\mathrm{HDL}$ increased the risk for having $\mathrm{HF}$ stage- $B$, which is in line with previous findings suggesting an important contribution for components of the MetS to HF. ${ }^{25,26}$ However, although hypertension is known to be a major cause of developing heart failure, ${ }^{27,28}$ the prevalence of $\mathrm{HT}$ in our former PE population with $\mathrm{HF}$ stage-B was comparable to the prevalence of hypertension in the non HF group. This can be explained by the inhibitory effect of certain antihypertensive medication (mainly ACE inhibitors and $\beta$-blockers) on cardiac remodeling. ${ }^{17}$ It is therefore conceivable that the comparable prevalence of hypertension in the non $\mathrm{HF}$ group is related to the use of antihypertensive medication. Interestingly, the prevalence of prehypertension amongst this group was high (36\%). Prehypertension is known to predispose to later clinical hypertension ${ }^{29}$ and is also associated 
cardiovascular morbidity. Formerly preeclamptic women with residual postpartum increase in LVM might be more sensitive to a slightly increased blood pressure as persistent prehypertension has been shown to accelerate the development of left ventricular hypertrophy. ${ }^{29,30}$ In our study, prehypertension increases the risk for having HF stage-B 3.9 fold. We therefore suggest considering intervention for prehypertension in formerly preeclamptic women especially with the co-occurrence of increased left ventricular mass and/ or concentric remodeling.

Despite advances in the diagnosis and treatment of HF, the number of women dying from HF increases annually. ${ }^{28}$ Early detection at the preclinical stages of HF and preventing the progression towards clinical stages is of great importance since this progression is associated with an increase in cardiovascular mortality, decreased health-related quality of life, and higher health care costs. ${ }^{18,19}$ Progression to the clinical stages of HF is preventable with relatively low costs interventions such as lifestyle adaptations and easily available medication. ${ }^{18}$ The first step in preventing the progression to the clinical stages of HF is early detection at the preclinical stages. This presents clinicians a challenge. First, HF stage-B is an asymptomatic stage which does not urge patients to seek medical care. Diagnosis could therefore easily be missed. Second, the overall prevalence of HF stage-B in middle aged women in the general population is very low, making screening programs for the whole female population of that age probably not cost-effective urging the need for identifying high risk subpopulations. Therefore, the clinical impact of our results is substantial as we now confirm the presence of a well-defined former preeclamptic population that is at a 10 times increased risk for having HF stage-B compared to the general female population of the same age. Our findings present clinicians the opportunity to early detect and intervene as to prevent further progression into the clinical stages of heart failure.

Phenotypic presentation of HF is currently split up between HF with reduced ejection fraction (HFrEF) and HF with preserved ejection fraction (HFpEF). Nowadays, HFpEF is considered a different phenotype within the HF spectrum, theoretically allowing a first stratification potential for clinical diagnosis and care of the HF syndrome. It is known from several studies that women are more likely to present with HFpEF than men. ${ }^{31-33}$ In our population, $76 \%$ of the formerly PE women with HF stage- B presented with preserved EF. As women tend to present CVD with atypical or nonspecific symptoms ${ }^{34}$, women with HF might already demonstrate cardiovascular symptoms without them being acknowledged as such. As a result, this could mean that formerly preeclamptic women might already have progressed to stage $\mathrm{C}$ without appropriate diagnosis or clinical management. Considering our data, it is therefore of upmost importance that $\mathrm{PE}$ is seen as a risk factor for HF and that a history of PE should be taken into account when assessing a woman's risk for CVD and HF. Additionally, clinicians need to be aware of gender differences in symptom presentation of cardiovascular diseases to early recognize CVD and HF in women.

Our study has several limitations that should be taken into account. Unfortunately, we were unable to classify $\mathrm{HF}$ in a few women due to missing values of the echocardiography in some cases. However, the unclassified subgroup did seem to differ from the classified group with respect to demography and baseline characteristics. Therefore, it is unlikely that excluding the unclassified group from analysis may have caused bias. Moreover, even with the missing values, the sample 
size was large enough to detect differences in the prevalence of HF stage-B. Another limitation is the fact that there were some differences in the baseline characteristics between the preeclamptic and control group, which could have led to confounding. There was a significant difference in the average age between the PE and the control group, with the control group being 4 years older. However, on the one hand the vascular ageing effect mainly confines to the postmenopausal period (35) and since all women were still premenopausal, it is unlikely that age differences influenced our results. On the other hand, despite the PE group being younger, we found a higher prevalence of HF in the PE group. Furthermore, in the multivariate analysis, we corrected for the variables that were significantly different between both groups keeping the possible confounding effect to a minimum.

In conclusion, we demonstrate an alarming higher incidence of asymptomatic stages of $\mathrm{HF}$ in formerly preeclamptic women compared to healthy parous controls. Since detecting the asymptomatic stages of HF enables clinicians to intervene in the progression towards more severe stages, we argue for more intensive postpartum screening in formerly preeclamptic women and recommend that after the postpartum cardiovascular screening, all formerly preeclamptic women are invited $<10$ years postpartum for a repeat cardiovascular risk evaluation.

\section{Acknowledgement}

We would like to thank Nicolette (Nicki) Breetveld and Veronica Lopes van Balen for their contribution in data collection,

\section{Perspectives}

Competency in medical knowledge: Preeclampsia has long term maternal cardiovascular implication.

Competency in patient care: Patients and clinicians should be aware of the importance of regular postpartum cardiovascular follow up after preeclampsia. When cardiovascular disease is diagnosed in an early stage, the patient may still benefit from secondary prevention.

Translational Outlook 1: Whether preeclampsia induces HF or whether preeclampsia and $\mathrm{HF}$ are a common result of the same susceptibility is yet to be unraveled.

Translational Outlook 2: The exact pathophysiological mechanism that contribute to the development of heart failure after preeclampsia is yet unknown. Exploring this mechanism may provide future perspectives, not only for understanding the link between both diseases but also to apply more tailored screening and prevention programs. 


\section{References}

1. WHO. Global status report on noncommunicable disaeses 2010.

2. Yusuf S, Reddy S, Ôunpuu S, Anand S. Global burden of cardiovascular diseases part I: general considerations, the epidemiologic transition, risk factors, and impact of urbanization. Circulation 2001;104:2746-2753.

3. Roger VL, Go AS, Lloyd-Jones DM et al. Executive Summary: Heart Disease and Stroke Statistics - 2011 Update A Report From the American Heart Association. Circulation 2011;123:459-463.

4. Perk J, De Backer G, Gohlke $\mathrm{H}$ et al. European Guidelines on cardiovascular disease prevention in clinical practice (version 2012). The Fifth Joint Task Force of the European Society of Cardiology and Other Societies on Cardiovascular Disease Prevention in Clinical Practice (constituted by representatives of nine societies and by invited experts). Eur Heart J 2012;33:1635-701.

5. Mosca L, Benjamin EJ, Berra $K$ et al. Effectiveness-based guidelines for the prevention of cardiovascular disease in women--2011 update: a guideline from the american heart association. Circulation 2011;123:1243-62.

6. Bellamy L, Casas JP, Hingorani AD, Williams DJ. Pre-eclampsia and risk of cardiovascular disease and cancer in later life: systematic review and metaanalysis. BMJ 2007;335:974.

7. Brown MC, Best KE, Pearce MS, Waugh J, Robson SC, Bell R. Cardiovascular disease risk in women with pre-eclampsia: systematic review and meta-analysis. Eur J Epidemiol 2013;28:1-19.

8. McDonald SD, Malinowski A, Zhou Q, Yusuf S, Devereaux PJ. Cardiovascular sequelae of preeclampsia/eclampsia: a systematic review and meta-analyses. Am Heart J 2008;156:918-30.

9. Fraser A, Nelson SM, Macdonald-Wallis C et al. Associations of pregnancy complications with calculated cardiovascular disease risk and cardiovascular risk factors in middle age: the Avon Longitudinal Study of Parents and Children. Circulation 2012;125:1367-80.

10. Brown MC, Best KE, Pearce MS, Waugh J, Robson SC, Bell R. Cardiovascular disease risk in women with pre-eclampsia: systematic review and meta-analysis. European journal of epidemiology 2013:1-19.

11. Brown MA, Lindheimer $M D$, de Swiet $M$, Van Assche $A$, Moutquin JM. The classification and diagnosis of the hypertensive disorders of pregnancy: statement from the International Society for the Study of Hypertension in Pregnancy (ISSHP). Hypertens Pregnancy 2001;20:IX-XIV.

12. Roberts JM, Cooper DW. Pathogenesis and genetics of pre-eclampsia. Lancet 2001;357:53-6.

13. Melchiorre K, Thilaganathan B. Maternal cardiac function in preeclampsia. Curr Opin Obstet Gynecol 2011;23:440-7.

14. Simmons LA, Gillin AG, Jeremy RW. Structural and functional changes in left ventricle during normotensive and preeclamptic pregnancy. Am J Physiol Heart Circ Physiol 2002;283:H1627-33. 
15. Melchiorre K, Sutherland GR, Baltabaeva A, Liberati M, Thilaganathan B. Maternal cardiac dysfunction and remodeling in women with preeclampsia at term. Hypertension 2011;57:85-93.

16. Melchiorre K, Sutherland GR, Liberati $M$, Thilaganathan B. Preeclampsia is associated with persistent postpartum cardiovascular impairment. Hypertension 2011;58:709-15.

17. Jessup M, Abraham WT, Casey DE et al. 2009 focused update: ACCF/AHA Guidelines for the Diagnosis and Management of Heart Failure in Adults: a report of the American College of Cardiology Foundation/American Heart Association Task Force on Practice Guidelines: developed in collaboration with the International Society for Heart and Lung Transplantation. Circulation 2009;119:1977-2016.

18. Ammar KA, Jacobsen SJ, Mahoney DW et al. Prevalence and prognostic significance of heart failure stages: application of the American College of Cardiology/American Heart Association heart failure staging criteria in the community. Circulation 2007;115:1563-70.

19. Desvigne-Nickens P. Heart Failure Prevention Is the Best Option To Stem High Costs and Disease Burden Research for More Effective Heart Failure Treatment Is Needed. Circulation: Cardiovascular Quality and Outcomes 2011;4:143-145.

20. Visser GH, Eilers PH, Elferink-Stinkens PM, Merkus HM, Wit JM. New Dutch reference curves for birthweight by gestational age. Early Hum Dev 2009;85:73744.

21. Report of the National High Blood Pressure Education Program Working Group on High Blood Pressure in Pregnancy. Am J Obstet Gynecol 2000;183:S1-S22.

22. Sahn DJ, DeMaria A, Kisslo J, A W. Recommendations regarding quantitation in m-mode echocardiography: Results of a survey of echocardiographic measurements. Circulation 1978:12.

23. Lang RM, Bierig $\mathrm{M}$, Devereux $\mathrm{RB}$ et al. Recommendations for chamber quantification: a report from the American Society of Echocardiography's Guidelines and Standards Committee and the Chamber Quantification Writing Group, developed in conjunction with the European Association of Echocardiography, a branch of the European Society of Cardiology. J Am Soc Echocardiogr 2005;18:1440-63.

24. Teichholz LE, Kreulen T, Herman MV, Gorlin R. Problems in echocardiographic volume determinations: echocardiographic-angiographic correlations in the presence of absence of asynergy. Am J Cardiol 1976;37:7-11.

25. Loehr LR, Rosamond WD, Poole C et al. Association of Multiple Anthropometrics of Overweight and Obesity With Incident Heart Failure The Atherosclerosis Risk in Communities Study. Circulation: Heart Failure 2009;2:18-24.

26. Ebong IA, Goff DC, Rodriguez CJ et al. The relationship between measures of obesity and incident heart failure: The multi-ethnic study of atherosclerosis. Obesity 2013.

27. Lloyd-Jones DM, Larson MG, Leip EP et al. Lifetime Risk for Developing Congestive Heart Failure: The Framingham Heart Study. Circulation 2002;106:3068-3072. 
28. Gordon T, Castelli WP, Hjortland MC, Kannel WB, Dawber TR. High density lipoprotein as a protective factor against coronary heart disease: the Framingham Study. The American journal of medicine 1977;62:707-714.

29. Ghossein-Doha C, Peeters L, van Heijster S et al. Hypertension After Preeclampsia Is Preceded by Changes in Cardiac Structure and Function. Hypertension 2013.

30. Markus MR, Stritzke J, Lieb $\mathbf{W}$ et al. Implications of persistent prehypertension for ageing-related changes in left ventricular geometry and function: the MONICA/KORA Augsburg study. J Hypertens 2008;26:2040-9.

31. Owan TE, Hodge DO, Herges RM, Jacobsen SJ, Roger VL, Redfield MM. Trends in prevalence and outcome of heart failure with preserved ejection fraction. New England Journal of Medicine 2006;355:251-259.

32. Bhatia RS, Tu JV, Lee DS et al. Outcome of heart failure with preserved ejection fraction in a population-based study. New England Journal of Medicine 2006;355:260-269.

33. O'Meara E, Clayton T, McEntegart MB et al. Sex differences in clinical characteristics and prognosis in a broad spectrum of patients with heart failure results of the Candesartan in Heart Failure: Assessment of Reduction in Mortality and Morbidity (CHARM) Program. Circulation 2007;115:3111-3120.

34. Dey S, Flather MD, Devlin $G$ et al. Sex-related differences in the presentation, treatment and outcomes among patients with acute coronary syndromes: the Global Registry of Acute Coronary Events. Heart 2009;95:20-26.

35. Schillaci G, Verdecchia P, Borgioni C, Ciucci A, Porcellati C. Early cardiac changes after menopause. Hypertension 1998;32:764-9. 



\section{General discussion and summary}

The objectives of the studies clustered in this thesis were a.) to explore in formerly early-onset PE patients, whether different preconceptional echocardiographic indices and/or abnormal cardiac adaptation to pregnancy precede recurrent PE in the next pregnancy (chapter 2); b.) to explore in these former patients, whether the agedependent changes in their cardiac geometry and function differ from those in healthy parous controls between 1 and 14 years postpartum (chapter 3); c.) to determine in normotensive, seemingly healthy, formerly PE women at one-year postpartum, whether the PE-induced increase in LVMi and other known risk factors relate to the premature development of chronic hypertension (chapters 4 \& 5), and finally, d.) to determine, whether at 5 to 7 years postpartum asymptomatic HF is more prevalent in women with a history of PE than in matched parous controls (chapter 6).

\section{Residual left ventricular remodeling (LVR) and recurrent PE}

Postpartum many former PE patients can be identified with structural cardiac abnormalities. The most relevant abnormality is residual concentric LVR. The latter is most prevalent after early-onset PE and may persist for an extended period of time. ${ }^{1}$ Pregnancy represents a physiologic stress model. Therefore, course and outcome of a next pregnancy in former PE patients can be expected to provide valuable information on the functional impact of this residual LVR on cardiovascular function (chapter 2).

In order to explore the functional impact of the observed abnormal geometric indices, we compared the echocardiographic indices of prepregnancy residual LVR between former PE patients, who did and did not develop recurrent PE in their next pregnancy (chapter 3 ). The results indicate that these two former patient subgroups elicited a similar pattern of cardiac adaptation to the next pregnancy. An unexpected finding was that PE recurrence clustered in former patients characterized by a relatively low pre-pregnancy LVMi and stroke volume, together with a relatively high heart rate and low E/A ratio. This observation provides indirect support for the view that apparently, a relatively small heart relative to body height represents a yet unknown risk factor for developing recurrent PE in a next pregnancy. Nevertheless, the effect of residual LVR in specific subgroups by universal "one-size-fits-all" criteria should be interpreted with caution, as these criteria have not been validated for our study population of former early-onset PE patients with a pre-existent relatively small heart. It is conceivable that these universal criteria are unsuitable to diagnose residual LVR in women with a relatively small heart. By including the diastolic function, it may be possible to overcome this potential diagnostic problem. At presence, we conclude that the functional relevance of a relatively small heart in relation to PE risk requires more study in a larger subgroup. 


\section{Potential risk factors for chronic HT after PE}

The findings in chapter 2 did not exclude the possibility that residual LVR relates to chronic HT later in life. Although PE had no appreciably effect on the (short-term) agedependent changes in cardiac geometry, this does not exclude the possibility of midand long-term functional cardiovascular sequels with respect to the risk of CVD and chronic HT.

In this context, we want to stress that untreated chronic HT by itself is a well-known cause of structural cardiovascular alterations and premature cardiovascular morbidity. ${ }^{2}$ Chronic HT is to be considered an important intermediate risk condition, which is not only easily identifiable but also modifiable enabling the timely institution of measures to prevent the premature development of more debilitating CVD in women. The pathogenesis of chronic HT in former PE patients is multifactorial. Our 6 to 12 months postpartum data (chapter $4 \& 5$ ) on the independent association of a raised LVMi with the development of chronic HT later in life in normotensive former patients was mixed: Although the results of our large cross-sectional study (chapter 4) suggested raised LVMi to be an independent risk marker for the development of remote chronic HT, our longitudinal pilot study over a 14-years postpartum period did not confirm this finding (chapter 5). This discrepancy may be related to the modest sample size which increases the type II error, along with the different composition of the study population in the latter study which - in contrast to the study population in chapter 4 consisted almost exclusively of women with a history of early-onset PE. In addition, as already mentioned before, identifying residual LVR is likely to be more difficult in former patients with a relatively small heart.

Another important finding in these two studies was the high prevalence of prehypertension at the one-year postpartum screening. In both studies these prehypertensive former patients tended to cluster in the subgroup of those normotensive former patients who developed chronic HT afterwards (chapters 4 and 5). As a consequence, we observed a prevalence of prehypertension of $66 \%$ in both chronic-HT subgroups, which is not only more than twice as high as that reported for the general population in the Netherlands, ${ }^{3}$ but also twice as high as that observed in the 2 subgroups of former patients remaining normotensive. Six years later, the subgroup of "normotensive" former patients with prehypertension, had developed chronic HT almost 4 times more often than their counterparts with a BP below that threshold. Together, these inferences support the concept that prehypertension relates to the later development of chronic HT with the observed residual LVR at oneyear postpartum.

In the studies clustered in this thesis the following potential contributors to the development of chronic HT later in life were identified one-year postpartum in normotensive seemingly-healthy, formerly-preeclamptic women.

1. Pre-hypertension, defined as systolic BP between 120 and $139 \mathrm{mmHg}$ and/or a diastolic BP between 80 and $89 \mathrm{mmHg}$, has been identified as a potent predictor of the development of chronic HT. ${ }^{4}$ Data from the Framingham Heart Study indicated that, compared to a blood pressure below $120 / 80 \mathrm{mmHg}$, prehypertension was associated with an increased risk of myocardial infarction (RR 3.5) and coronary 
artery disease (RR 1.7)..$^{5}$ Unfortunately, prehypertension is not yet generally accepted as a serious health problem, even though it tends to coincide with traditional cardiovascular risk factors., 7 In our studies, we confirmed prehypertension to be an important "background" risk factor for developing chronic $\mathrm{HT}$ after PE.

2. PE-induced increase in LVMi. In our large cross-sectional study (chapter 4), we noticed that normotensive former patients who developed chronic HT, had a raised LVMi. The Framingham study provided evidence for a correlation between increased LVMi and the subsequent development of chronic HT in previously normotensive subjects, but the data in that study were unsuitable to prove causality. ${ }^{8}$ It is conceivable that some genetic susceptibility in the presence of relevant environmental risk factors (sedentary lifestyle, chronic exposure to intoxications and specific deficiencies, and unhealthy eating habits) may contribute to both the pathogenesis of LVMi and that of chronic HT. ${ }^{9,10,11}$

3. Components of the MetS. The metabolic syndrome (MetS) is another well-known contributor to the development of chronic $\mathrm{HT}^{12-14}$ Our longitudinal pilot study in initially normotensive formerly early-onset PE patients (chapter 5) provides only modest evidence for raised fasting insulin levels one-year postpartum to contribute to a higher risk of developing chronic HT later in life, most likely due to the modest power of that study. Nevertheless, the reported evidence for MetS being a contributor to the development of both PE and subsequently chronic HT is overwhelming, $^{12,13,15}$ with MetS probably contributing independently to the increased risk of developing chronic HT after PE. ${ }^{16-18}$

On the basis of these observations, three interpretations regarding the development of chronic HT later in life in former patients are plausible: 1.) Former patients with prehypertension are at increased risk to develop chronic HT with LVH already developing during prehypertension. 2.) LVH and chronic HT have overlapping pathogeneses with typical risk factors such as the cardio-metabolic constitutes of MetS, expediting the onset of clinical symptoms. 3.) Primary LVH may develop in the presence of a susceptible genetic make-up and predisposes to chronic HT.19 In this context it is relevant to emphasize that PE in all three scenarios is a superimposed pregnancy complication. Our research data are unsuitable to determine whether PE contributes independently to the development of chronic HT later in life.

\section{Cardiac aging and asymptomatic Heart Failure after $P E$}

Many women develop echocardiographic abnormalities during the clinical phase of PE. In almost half of the cases, these structural abnormalities persist for more than one year postpartum, particularly after early-onset PE. ${ }^{1}$ When using the algorithm proposed by the AHA to classify asymptomatic HF in our case-control study (Chapter 6 ), we found that the prevalence of the pre-clinical stage-B HF is about 4 times higher in women with a history of PE (21\%) than in controls (6\%). Moreover, PE seems to be an independent risk factor for stage-B HF with an adjusted odds ratio of 11 . It seems that the reported high prevalence of stage-B HF at one-year postpartum indicates a protracted resolving process, with often even persistent residual LVR in many former 
patients. From the former PE women who had stage-B HF, 76\% had a preserved ejection fraction, which is recently acknowledged as a gender-specific phenotype of $\mathrm{HF}^{20,21}$ Most women with stage-B HF in this group had mainly concentric LVR (64\%). However, although $\mathrm{HT}$ is known to be a major precursor of $\mathrm{HF}^{22,23}$ the prevalence of $\mathrm{HT}$ in our former PE population with stage-B HF was comparable to that in the non-HF group. The prevalence of HT may be influenced by the use of certain antihypertensive drugs (mainly ACE inhibitors and $\beta$-blockers) which are claimed to redress cardiac remodeling. ${ }^{24}$ Another interesting but worrisome finding was that the prevalence of HT in the non-HF subgroup of former patients was relatively high (36\%). This could indicate that a large proportion of seemingly healthy women, probably with mostly borderline $\mathrm{HT}$, remain untreated. In our study, women with prehypertension have an almost 4 times higher risk of having developed stage-B HF. This supports our own observation that prehypertension is an independent risk factor for stage-B HF in former PE patients.

\section{Cardiovascular risk management}

Although women with a history of PE are at increased risk of premature CVD later in life, there is no consensus - and thus no guideline - about how to screen or manage these women. ${ }^{25}$ Although we know that certain preexisting risk factors predispose to, or are associated with the development of PE in the first pregnancy, until now preconceptional care has not been incorporated in our preventive programs. Meanwhile, the timing of a post-PE screening one-year postpartum, appears to be appropriate as we noticed in our studies and in reports by others, ${ }^{1,25,26}$ that at oneyear postpartum, the majority of functionally relevant cardiovascular changes induced by the preceding PE will have subsided in most (but not all) former patients. Conversely, postponing post-PE-screening to a later date to determine with more certainty that the detected cardiovascular changes are persistent, increases the risk for loss to follow-up and may depreciate the preconceptional counseling for a pending next pregnancy.

Unfortunately, until now, there is no experimental evidence supporting the concept that antihypertensive drugs reduce the risk of prehypertension to evolve to chronic HT in otherwise healthy women. ${ }^{27}$ Nevertheless, in patients without diabetes, chronic kidney disease, or any other clinically relevant end-organ damage caused by cardiovascular disease, lifestyle adjustments consisting of weight reduction, sodium restriction, increased physical activity, avoidance of excess alcohol, ${ }^{28}$ and stress management ${ }^{29}$ can be expected to ameliorate cardiovascular health.

Our findings stress the need for a postpartum cardiovascular risk assessment for all women with a history of PE as recently proposed ${ }^{31}$, with primary focus on screening former early-onset PE patients and special emphasis on the presence of prehypertension. The results of these exams should be used to counsel these women, providing them with a clear, but also feasible long-term risk management advice. These relatively young women are at high risk of developing chronic HT triggering concentric LVR in the subsequent decade. 
The findings presented in this thesis do not differentiate between PE being an independent risk factor for chronic HT later in life, or merely a "wake-up call" alerting the affected woman for reduced cardiovascular reserves that predispose her to premature CVD. ${ }^{30}$ Therefore, the observations in this thesis, together with the available evidence reported in the literature indicate that in most women, PE is a serious clinical event, which does not always resolve in a period of weeks to months postpartum leaving behind persistent residual structural and functional damage of the maternal cardiovascular system. That is to say, the increased risk of chronic HT and premature CVD after PE appears to be primarily related to subclinical cardiovascular risk factors (mostly prehypertension, LVR and features of the metabolic syndrome). Nevertheless, it is still unsettled whether 1.) PE in the absence of other risk factors also predisposes independently to chronic HT and CVD, and 2.) PE magnifies the negative effects of other subclinical risk factors on cardiovascular health, thus expediting the development of premature CVD.

In conclusion: Although our findings point toward a consistent post-PE cardiovascular response pattern, some of our data should be interpreted with caution in conjunction with the modest sample sizes and thus limited statistical power. Nevertheless, the studies in this thesis provide experimental evidence for a high prevalence of prehypertension, persistent CLVH and features of the metabolic syndrome in former PE patients at one year postpartum. In the studies included in this thesis, we demonstrate and/or confirm that these conditions are associated with recurrent $\mathrm{PE}$, chronic HT and stage-B HF later in life. PE should alert the clinician providing care to such a patient for the possibility to offer her a cardiovascular risk assessment one-year postpartum with at least regular BP checkups, enabling early secondary prevention.

Scientific perspective. The scientific perspectives of this thesis lead us to the rationale behind the Queen of Hearts research program. The Queen of Hearts study aims to improve diagnosis of cardiovascular disease in women in general, and in formerly preeclamptic women in particular. This new research program extends CV phenotyping of high-risk women to allow early identification in a preclinical stage of CVD. These preclinical stages are known to be at least partlyreversible. Slowing progression may improve their cardiovascular health at later age and with it, survival rate. Besides CV phenotyping, biomarkers will be explored not only intended to improve early diagnosis, but also to improve understanding of the pathogenesis of -PE-related CVD. 


\section{References}

1. Melchiorre K, Sutherland GR, Liberati M, Thilaganathan B. Preeclampsia is associated with persistent postpartum cardiovascular impairment. Hypertension. 2011;58:709-715

2. Davila DF, Donis JH, Odreman R, Gonzalez M, Landaeta A. Patterns of left ventricular hypertrophy in essential hypertension: Should echocardiography guide the pharmacological treatment? International journal of cardiology. 2008;124:134-138

3. Agyemang C, van Valkengoed I, van den Born BJ, Stronks K. Prevalence and determinants of prehypertension among african surinamese, hindustani surinamese, and white dutch in amsterdam, the netherlands: The sunset study. European journal of cardiovascular prevention and rehabilitation : official journal of the European Society of Cardiology, Working Groups on Epidemiology \& Prevention and Cardiac Rehabilitation and Exercise Physiology. 2007;14:775-781

4. Parikh NI, Pencina MJ, Wang TJ, Benjamin EJ, Lanier KJ, Levy D, D'Agostino RB, Sr., Kannel WB, Vasan RS. A risk score for predicting near-term incidence of hypertension: The framingham heart study. Annals of internal medicine. 2008;148:102-110

5. Qureshi Al, Suri MF, Kirmani JF, Divani AA, Mohammad Y. Is prehypertension a risk factor for cardiovascular diseases? Stroke. 2005;36:1859-1863

6. Pletcher MJ, Bibbins-Domingo K, Lewis CE, Wei GS, Sidney S, Carr JJ, Vittinghoff E, McCulloch CE, Hulley SB. Prehypertension during young adulthood and coronary calcium later in life. Annals of internal medicine. 2008;149:91-99

7. Greenlund KJ, Croft JB, Mensah GA. Prevalence of heart disease and stroke risk factors in persons with prehypertension in the united states, 1999-2000. Arch Intern Med. 2004;164:2113-2118

8. Lauer MS, Anderson KM, Levy D. Influence of contemporary versus 30-year blood pressure levels on left ventricular mass and geometry: The framingham heart study. J Am Coll Cardiol. 1991;18:1287-1294

9. Wang $X$, Prins BP, Sober S, Laan M, Snieder H. Beyond genome-wide association studies: New strategies for identifying genetic determinants of hypertension. Curr Hypertens Rep. 2011;13:442-451

10. Greenwood JP, Scott EM, Stoker JB, Mary DA. Hypertensive left ventricular hypertrophy: Relation to peripheral sympathetic drive. J Am Coll Cardiol. 2001;38:1711-1717

11. Schlaich MP, Kaye DM, Lambert E, Sommerville M, Socratous F, Esler MD. Relation between cardiac sympathetic activity and hypertensive left ventricular hypertrophy. Circulation. 2003;108:560-565

12. Barden AE, Beilin LJ, Ritchie J, Walters BN, Michael C. Does a predisposition to the metabolic syndrome sensitize women to develop pre-eclampsia? J Hypertens. 1999;17:1307-1315

13. Solomon CG, Seely EW. Brief review: Hypertension in pregnancy : A manifestation of the insulin resistance syndrome? Hypertension. 2001;37:232239 
14. Harskamp RE, Zeeman GG. Preeclampsia: At risk for remote cardiovascular disease. Am J Med Sci. 2007;334:291-295

15. Strobl I, Windbichler G, Strasak A, Weiskopf-Schwendinger V, Schweigmann U, Ramoni $A$, Scheier $M$. Left ventricular function many years after recovery from pre-eclampsia. BJOG. 2011;118:76-83

16. Zandstra $M$, Stekkinger $E$, van der Vlugt $M J$, van Dijk AP, Lotgering FK, Spaanderman ME. Cardiac diastolic dysfunction and metabolic syndrome in young women after placental syndrome. Obstet Gynecol. 2010;115:101-108

17. Spaan JJ, Sep SJ, van Balen VL, Spaanderman ME, Peeters LL. Metabolic syndrome as a risk factor for hypertension after preeclampsia. Obstet Gynecol. 2012;120:311-317

18. Scioscia M, Gumaa K, Rademacher TW. The link between insulin resistance and preeclampsia: New perspectives. J Reprod Immunol. 2009;82:100-105

19. Post WS, Larson MG, Levy D. Impact of left ventricular structure on the incidence of hypertension. The framingham heart study. Circulation. 1994;90:179-185

20. Owan TE, Hodge DO, Herges RM, Jacobsen SJ, Roger VL, Redfield MM. Trends in prevalence and outcome of heart failure with preserved ejection fraction. N Engl J Med. 2006;355:251-259

21. O'Meara E, Clayton T, McEntegart MB, McMurray JJ, Pina IL, Granger CB, Ostergren J, Michelson EL, Solomon SD, Pocock S, Yusuf S, Swedberg K, Pfeffer $M A$, Investigators $C$. Sex differences in clinical characteristics and prognosis in a broad spectrum of patients with heart failure: Results of the candesartan in heart failure: Assessment of reduction in mortality and morbidity (charm) program. Circulation. 2007;115:3111-3120

22. Lloyd-Jones DM, Larson MG, Leip EP, Beiser A, D'Agostino RB, Kannel WB, Murabito JM, Vasan RS, Benjamin EJ, Levy D. Lifetime risk for developing congestive heart failure: The framingham heart study. Circulation. 2002;106:3068-3072

23. Gordon T, Castelli WP, Hjortland MC, Kannel WB, Dawber TR. High density lipoprotein as a protective factor against coronary heart disease: The framingham study. The American journal of medicine. 1977;62:707-714

24. Jessup M, Abraham WT, Casey DE, Feldman AM, Francis GS, Ganiats TG, Konstam MA, Mancini DM, Rahko PS, Silver MA, Stevenson LW, Yancy CW. 2009 focused update: Accf/aha guidelines for the diagnosis and management of heart failure in adults: A report of the american college of cardiology foundation/american heart association task force on practice guidelines: Developed in collaboration with the international society for heart and lung transplantation. Circulation. 2009;119:1977-2016

25. Spaan J, Peeters L, Spaanderman M, Brown M. Cardiovascular risk management after a hypertensive disorder of pregnancy. Hypertension. 2012;60:1368-1373

26. Firoz T, Melnik T. Postpartum evaluation and long term implications. Best practice \& research. Clinical obstetrics \& gynaecology. 2011;25:549-561

27. Schunkert H. Pharmacotherapy for prehypertension--mission accomplished? $\mathrm{N}$ Engl J Med. 2006;354:1742-1744

28. Chobanian AV, Bakris GL, Black HR, Cushman WC, Green LA, Izzo JL, Jr., Jones DW, Materson BJ, Oparil S, Wright JT, Jr., Roccella EJ. Seventh report of the joint 
national committee on prevention, detection, evaluation, and treatment of high blood pressure. Hypertension. 2003;42:1206-1252

29. von Kanel R. Psychosocial stress and cardiovascular risk : Current opinion. Swiss medical weekly. 2012;142:w13502

30. Sattar N, Greer IA. Pregnancy complications and maternal cardiovascular risk: Opportunities for intervention and screening? Bmj. 2002;325:157-160

31. Richtlijn Cardiovasculair risicomanagement na een reproductieve aandoening; Nederlandse Vereniging voor Obstetrie en Gynaecologie; 2014 


\section{Summary}

This thesis describes a number of studies related to long-term consequences of preeclampsia (PE) on cardiovascular health. Chapter $\mathbf{1}$ presents the problems underlying the research questions explored in this thesis. Pregnancy can be seen as a woman-specific stress test for the cardiovascular system. Hypertensive complications during pregnancy consist as maternal symptoms (e.g. de-novo hypertension with or without abnormal proteinuria) usually in concert with signs of placental insufficiency (e.g. fetal growth restriction). Women experiencing a hypertensive pregnancy disorder have a 2-8 times higher risk of developing cardiovascular disease (CVD) later on in life. PE and CVD share various risk factors such as obesity, hypertension and diabetes mellitus, which suggests common etiological pathways. During PE, the heart remodels abnormally, compared to cardiac remodeling in normotensive pregnancy. During an uneventful pregnancy the heart becomes hypertrophic, however, with balanced increases in left ventricular mass (LVM) and left ventricular diameter. This adaptation is called "eccentric cardiac remodeling". During PE, LVM increases additionally, but without concomitant widening of the left ventricular diameter, so-called "concentric cardiac remodeling". This type of cardiac remodeling occurs also in Heart Failure (HF).

This thesis consists of two parts. In the first part, we explored in former PE patients the cardiac adaptive response to the next pregnancy and secondly, the age-related change in cardiac geometry over a period of 14 years. Chapter $\mathbf{2}$ describes the results of a study group consisting of formerly early-onset PE patients. We studied the research question, whether recurrent PE in the next pregnancy is preceded by more abnormal preconceptional cardiac ultrasound indices and/or by an abnormal initial pattern of cardiac adaptation to pregnancy. Post hoc, women were divided in a subgroup who developed recurrent PE and a subgroup with a second normotensive pregnancy. In this chapter we showed that women who had developed recurrent PE differ in their next pregnancy from their counterparts with a normotensive next pregnancy, by a lower LVM along with a smaller stroke volume and a higher heart rate. This difference persisted until the $20^{\text {th }}$ week of gestation. Chapter 3 presents the age-related changes in cardiac geometry and function in former PE patients relative to parous controls between 1 and 14 years postpartum. In this chapter, we demonstrate that the agerelated cardiac adaptation in former PE patients does not differ appreciably from that in parous controls. However, this study was performed without taking into account that some normotensive women at 1 year postpartum had developed chronic hypertension in the subsequent 13 years preceding the measurement session at 14 years postpartum.

In the second part, we explored in former PE patients, to what extent the presence of a number of cardiovascular risk factors at 1 year postpartum relate to the development of chronic HT and asymptomatic HF (HF stage-B), later in life.

Chapter 4 evaluates in a group of women with a history of $\mathrm{PE}$, whether CV risk factors and cardiac indices are associated with the development of hypertension in initially normotensive women. Former PE women tend to differ from normal parous controls by an increased LVM index, a higher diastolic blood pressure and a higher incidence of 
prehypertension, features which are strongly associated with the development of chronic hypertension within the next decade.

Chapter 5 presents a longitudinal pilot study in women with a history of PE. We performed cardiovascular and metabolic screening at 1 and 14 years postpartum. At 14 years postpartum, we divided women into a subgroup who had developed chronic hypertension and a subgroup who remained normotensive. We demonstrated that women with hypertension at 14 years postpartum had already prehypertension at 1 year postpartum. Moreover, together with the chronic hypertension these women had developed a higher relative left-ventricular wall thickness and a lower diastolic function at 14 years postpartum. Chapter 6 shows the results of a cross-sectional cohort study in which we show that in the first 10 post-PE years one out of four women could be identified with asymptomatic HF stage-B. HF is a major cause of mortality in women. The progression from the preclinical HF-B to the clinical HF-C is accompanied by a 5 -fold rise in mortality. In this chapter we show that a mildly increased blood pressure, a higher BMI and a lower HDL are associated with this asymptomatic stage. In Chapter $\mathbf{7}$ we elaborate on the main findings of this thesis. The studies in this thesis in former PE patients provide experimental evidence for a high prevalence of prehypertension, persistently raised LVM and features of the metabolic syndrome at one year postpartum. In the studies included in this thesis, we demonstrate that these conditions are associated with chronic HT and HF stage-B later in life. PE should alert the clinician providing care to such a patient for the possibility to offer her a cardiovascular risk assessment at one-year postpartum with at least regular BP check-ups, enabling the institution of timely secondary prevention.

Finally, Chapter 8 elaborates on the valorization possibilities of the study results, the explored cohorts and the ideas that emerged in the course of these studies. PE can be seen as a woman-specific risk indicator for CVD that provides the possibility to identify high-risk women at an early stage in life. A multidisciplinary cardiovascular risk assessment along with offering early secondary prevention is expected to be highly beneficial for this high-risk population. Because this group of women has a high prevalence of subclinical HF early in life, this subgroup offers the possibility to also explore for potentially attractive diagnostic biomarkers for HF, which may improve prediction and diagnosis. 




\section{Valorisatie}

Dit hoofdstuk beschrijft de waarde die gecreëerd kan worden uit de resultaten van de studies in dit proefschrift en hoe deze vertaald kunnen worden naar de klinische praktijk, het breder publiek en welke maatschappelijke belangen hiermee behartigt worden.

\section{Inleiding}

De zwangerschap wordt gezien als een vrouw-specifieke cardiovasculaire (CV) stress test. Vasculaire complicaties tijdens de zwangerschap uiten zich vaak via een hypertensief beeld, doorgaans in combinatie met een tekortschietende placentafunctie. Deze laatste brengt dan weer extra gezondheidsrisico's voor het ongeboren kind met zich mee. De kans op hart- en vaatziekten (HVZ) op middelbare leeftijd is bij een vrouw met dit type zwangerschapscomplicaties in de voorgeschiedenis twee tot acht maal verhoogd. Hoewel de bloeddruk postpartum doorgaans binnen twee weken normaliseert, blijven de onderliggende, meestal latente afwijkingen, vaak gewoon bestaan. Deze zijn aanvankelijk niet alleenmaar goed medicamenteus corrigeerbaar, maar een dergelijke correctie verlaagt ook nog eens de kans op blijvende cardiovasculaire restschade. Dit impliceert dat het achterwege laten van deze correctie ertoe leidt dat de initieel latente afwijkingen binnen 10 tot 20 jaar kunnen evolueren tot irreversibele hartvaatschade.

De focus van de studies in dit proefschrift, was met name het in kaart brengen van de vroege en late gevolgen van het doormaken van pre-eclampsie (PE) voor de structuur en functie van het hart van de vrouw. PE kan gezien worden als een vrouw-specifieke risico-indicator die op vroeg-volwassen leeftijd de mogelijkheid biedt om vrouwen met een verhoogd risico op HVZ op te sporen. De resultaten van de studies in dit proefschrift ondersteunen het concept dat de prevalentie van subklinisch hartfalen en van prehypertensie (mild verhoogde bloeddruk) bij deze groep van vrouwen verhoogd is.

\section{Relevantie}

Tussen juni 2012 en mei 2013 werden er in Nederland 172.000 kinderen geboren. Daarbij raakte bij ongeveer 11.000 vrouwen (6,4\%) de zwangerschap gecompliceerd door PE. Ofschoon het voor een klein deel een recidief betreft, kan toch geschat worden dat er jaarlijks zo'n 11.000 (nieuwe) vrouwen met een verhoogd risico op HVZ bijkomen.

De resultaten van de studies in dit proefschrift zijn op een aantal fronten relevant. Zij zorgen enerzijds voor extra aandacht voor een grote groep van jonge, meestal gezonde moeders die een pre-eclamptische zwangerschap hebben doorgemaakt en daarom met veel vragen zitten over hun gezondheid in het algemeen en dat van hun hartvaatbed in het bijzonder. Anderzijds krijgt het exploreren van de mogelijkheden tot preventie van vrouw-specifieke HVZ extra aandacht, een actueel onderwerp gezien de relatief hoge prevalentie van sterfte aan HVZ bij vrouwen die momenteel zelfs 
hoger is dan bij mannen. Door deze twee aspecten ontstaat er steeds meer behoefte om HVZ bij vrouwen beter te begrijpen, deze aandoeningen in een vroege fase te kunnen diagnosticeren en de link met PE beter te begrijpen. Een beter inzicht in vrouwspecifieke oorzaken van het ontwikkelen van HVZ en in hoe PE en HVZ onderling gerelateerd zijn maakt het mogelijk om preventie-op-maat in een vroeg stadium te ontwikkelen en aan te bieden. Ook de Nederlandse Hartstichting (NHS) heeft de relevantie van dit onderwerp opgemerkt en nadat decennialang mannen centraal stonden binnen het onderzoek naar HVZ, is dit belangrijke agendapunt door de enquête van de NHS verkozen tot nummer 2 op de prioriteitenlijst voor wetenschappelijk onderzoek direct voorafgegaan door het eerder herkennen van HVZ dat op nummer 1 staat. Om hieraan tegemoet te komen steunt de NHS onder andere ons expertise centrum voor vrouwen met gestationele hypertensieve aandoeningen door subsidiering van de "Queen of Hearts" studie die zich tot doel stelt om beter inzicht te verkrijgen en potentiele biomarkers te vinden voor de relatie tussen HVZ en PE.

De resultaten in dit proefschrift tonen aan dat bij vrouwen met PE in de anamnese het hart in de daaropvolgende 14 jaar zich op verschillende manieren kan aanpassen. Het ontwikkelen van hypertensie na pre-eclampsie blijkt hierbij de belangrijkste factor te zijn. Het was daarbij met name opvallend dat ook een hoog-normale bloeddruk, zg. "prehypertensie" niet alleen het risico op chronische hypertensie verhoogt, maar ook een ongunstig effect heeft op de aanpassing van het hart na PE. Deze resultaten ondersteunen de relevantie van strikte, regelmatige bloeddrukcontrole bij deze groep vrouwen vanaf de bevalling.

Omdat deze populatie van vrouwen relatief groot is, en de risico's pas nauwkeurig ingeschat kunnen worden als er een risicomodel over meerdere jaren beschikbaar is, is het belangrijk om de risicostratificatie in kwantitatieve risico's aan vrouwen te kunnen communiceren. Dit is tot op heden nog niet mogelijk.

\section{Doelgroep}

De studies in dit proefschrift zijn uitgevoerd bij jonge, ogenschijnlijk gezonde vrouwen die PE doormaakten. Bij deze doelgroep werd onderzoek gedaan naar vroege, intermediaire vormen van HVZ zoals prehypertensie, hypertensie en hartfalen (HF) stadium B. Door de relatief jonge leeftijd van deze populatie, is er met vroegtijdige screening - en preventieprogamma's nog veel winst te behalen op het gebied van leefstijl-aanpassingen en andere niet-medicamenteuze preventieve interventies. Daarnaast is HF-B een subklinisch en veelal reversibele vorm van HF. HF is de primaire oorzaak van CV geralteerde hospitalisatie en sterfte in Westerse landen. Als HF eenmaal is vastgesteld, hebben patiënten doorgaans intensieve behandeling nodig in combinatie met frequent ziekenhuisbezoek. In 2012 werden in Nederland 130.000 patiënten met HF gediagnostiseerd. Daarnaast overlijden dagelijks in Nederland 107 mensen met HF waarvan meer dan de helft vrouw is. Omdat de algemene populatie steeds ouder wordt zal de verwachte prevalentie van HF met $50 \%$ stijgen in de periode 2005-2025. De jaarlijkse kosten voor HF in Nederland bedragen ongeveer $€ 450$ miljoen (2007). 


\section{Onderzoeksresultaten en mogelijke consequenties voor de zorg (Activiteiten en producten)}

\section{Multidisciplinaire cardiovasculair risicomanagement}

De cohorten die in dit proefschrift voor statistische analyse werden gebruikt, kwamen tot stand door het reeds 20 jaar bestaande beleid in het MUMC+ en in het Radboud $\mathrm{UMC}+$ om CV risicomanagement (CVRM) toe te passen bij vrouwen met PE in de voorgeschiedenis.

Tot enige jaren geleden was er in de medische wereld weinig affiniteit en dus ook geen richtlijn met betrekking tot deze problematiek. De huidige zorg aan zwangeren met een hypertensieve aandoening wordt nog bijna overal primair verleend door de obstetricus die die zorg vaak 6 weken na de bevalling afsluit. De cardioloog ziet de vrouw pas bij manifeste cardiale morbiditeit. Daarnaast is het conventionele CVRM door de vasculaire internist nog niet zodanig georganiseerd dat voormalige PE patiënten daar makkelijk aansluiting bij kunnen vinden. Dit leidt ertoe dat de zorg voor de niet zwangere vrouw met meestal niet-klassieke risicofactoren en zonder manifeste CV-ziekte, medisch tussen wal en schip valt. Binnen de obstetrie wordt deze weeffout in de preventieve cardiovasculaire zorg gevoeld. De obstetricus ziet immers al vroeg in het leven van de vrouw, tijdens de zwangerschap, of de vrouw over de reserves beschikt die nodig zijn om de hemodynamische belasting van de zwangerschap te kunnen dragen. Deze reserves worden ook aangesproken naarmate een vrouw ouder wordt. Helaas, verliest menig obstetricus deze vrouw na de bevalling uit het oog, waardoor het ooit opgemerkte verhoogde CV risico uit beeld raakt. Een multidisciplinair zorgtraject waarin gestandaardiseerde CVRM wordt aangeboden met bijbehorende follow-up kan de CV zorg aan vrouwen in het algemeen en vrouwen na een pre-eclamptische zwangerschap in het bijzonder verbeteren. De resultaten uit dit proefschrift met betrekking tot de relatie tussen prehypertensie en een vergroot hart enerzijds en het ontwikkelen van hoge bloeddruk anderzijds zouden direct geïmplementeerd kunnen worden. Ook is het nu al mogelijk preventieve strategiën te ontwikkelen op basis van de gevonden verhoogde prevalentie van HF-B bij vrouwen met obesitas, een verlaagde HDL cholesterol en/of prehypertensie.

\section{Biomarkers}

Hartklachten worden vaak door de vrouw niet als zodanig herkend en worden door artsen vaak bestempeld als aspecifieke klachten. Ook zijn de huidige biomarkers voor diagnostiek van HVZ bij vrouwen beperkt gevoelig. Daarom word de diagnose van een cardiale aandoening, zeker bij relatief jonge vrouwen, vaak pas in een laat stadium gesteld. Een relatief late diagnose impliceert dat de behandeling moeilijker zal zijn, mede ook omdat het (reversibele) latente stadium dan meestal al gepasseerd is.

$\mathrm{HF}$ is doorgaans een geleidelijk progressieve verslechtering van de pompfunctie van het hart en wordt gekenmerkt door aanvankelijk een jarenlange klachtenvrije periode voorafgaande aan de ontwikkeling van een typisch klinisch beeld. Deze lange latente periode wordt door de American Heart Association (AHA) gedefinieerd als HF-B. Uit de resultaten van dit proefschrift, blijkt dat 1 op de 4 vrouwen met een PE in de voorgeschiedenis op relatief jonge leeftijd HF-B ontwikkelt. De afwezigheid van 
klachten maakt het moeilijk om HF-B te diagnosticeren. Toch is het stellen van de diagnose HF-B in dit stadium van groot belang, omdat de progressie naar stadium-C (HF met klachten) gepaard gaat met een 5 keer verhoogde mortaliteit. Behandeling van HF-B met $\beta$-blokkers dan wel ace-remmers verlaagt de mortaliteit met ongeveer $40 \%$ en vertraagt verdere progressie naar HF-C met ongeveer $30 \%$. Daarnaast zorgt het toedienen van de juiste medicatie bij HF-B tot een klinisch-relevante regressie van de structurele cardiale afwijkingen. Dit laatste onderstreept dat detectie en vervolgens tijdige en adequate behandeling van HF-B grote gezondheidswinst oplevert. Helaas schiet de huidige diagnostiek van HF-B vaak tekort (zie boven). Omdat veel vrouwen met HF-B daarom niet tijdig opgespoord worden, is het vaststellen en vervolgens behandelen van HF-B bij vrouwen nog steeds problematisch. Onze cohorten bieden de mogelijkheid om betere (gevoeligere) biomarkers voor HF-B te vinden specifiek voor onze doelgroep van hoog-risico vrouwen. Het vinden van een betrouwbare biomarker kan de opmaat vormen voor de betere identificatie van vrouwen met HF-B binnen deze risicopopulatie en bijdragen tot de ontwikkeling van preventieve strategieën die de progressie van HF-B naar HF-C tenminste vertragen;

Het verder in kaart brengen van de toepassingsmogelijkheden van zwangerschap als kortdurende fysiologische belastingstest voor het moederlijke cardiovasculaire systeem.

De normale en preeclamptische zwangerschap bieden de mogelijkheid om respectievelijk, de fysiologische en pathofysiologische aanpassingen van het hart van de vrouw over een langere periode te bestuderen. Dit biedt de mogelijkheid om een beter inzicht te krijgen, enerzijds in de grootte van haar cardiovasculaire reserves en anderzijds in de wijze waarop zij haar cardiale reserves aanwendt om een fysiologische belasting op te vangen. Deze informatie kan van pas komen ter bepaling van haar risico's op cardiale morbiditeit bij het klimmen der jaren. Uit één van de studies uit dit proefschrift blijkt er grote cardiale heterogeniteit te bestaan bij vrouwen die een PE hebben doorgemaakt. Zo viel op dat een deel van die vrouwen een opvallend klein hart had. Dit is een valkuil voor de definitie van LV hypertrofie bij vrouwen met PE in de voorgeschiedenis. De uitkomsten van dit proefschrift maken het aannemelijk dat er bij de definitie van cardiale remodelering bij vrouwen rekening gehouden moet worden met de preëxistente grootte van het moederlijk hart.

\section{Planning en Realisatie}

Kortgeleden zijn wij met een grootschalige studie gestart (de Queen of Hearts studie) die beoogt de diagnostiek van HVZ bij vrouwen te verbeteren. In deze studie zal een groot cohort van vrouwen met een PE in de voorgeschiedenis al dan niet met herkenbare klachten, gescreend worden op cardiovasculaire fitheid. Het is aannemelijk dat bij een aanzienlijk deel HF-B vastgesteld zal worden. Voor dit project gaan we 2580 vrouwen uitgebreid de cardiovasculaire structuur en functie in kaart brengen. Voor de genotypering werken wij samen met 4 andere universitaire centra om naar biomarkers te zoeken die ons kunnen helpen HF-B beter te diagnosticeren, maar ook om de pathogenese van HF-B op te helderen. 
Deze aanvraag levert, naast nieuwe biomarkers, ook informatie op over hoe zich diastolische dysfunctie bij vrouwen ontwikkelt. Voor dit onderzoek werkt de afdeling verloskunde nauw samen met de afdelingen cardiologie en vasculaire geneeskunde. Ons inziens leent het MUMC zich bij uitstek voor het screenen van deze populatie vanwege:

1. De sinds 1996 opgebouwde expertise ten aanzien van de zorg voor vrouwen met een hypertensieve zwangerschapscomplicatie (al dan niet in de anamnese) en waaruit de verschillende cohorten ontleend zijn die de basis vormden voor de studies in dit proefschrift.

2. Door de obstetrie geïnitieerde en al meer dan 20 jaar bestaande samenwerking met de afdelingen cardiologie en vasculaire geneeskunde.

3. De integratie van zorg, wetenschap en onderwijs op het innovatieve "transmuraal vrouwen dagcentrum".

Daarbij beschikt het MUMC+ sinds 1 oktober 2012 als eerste ziekenhuis ter wereld over een Hart-en Vaat centrum, waar zes medische specialismen onder één dak zijn samengebracht. Ook wordt er momenteel een cohort opgebouwd van patiënten met een vorm van HF zonder afname van de ejectiefractie. Deze vorm van HF komt het meest voor bij vrouwen. Daarnaast werd in de zomer van 2014 ons transmuraal vrouwen dagcentrum geopend waarin CVRM aan zwangere en niet-zwangere vrouwen wordt aangeboden. Ook zal de hieraan gekoppelde CV-multidisciplinaire poli van start gaan, waarin vrouwen met een hypertensieve zwangerschapscomplicatie in de voorgeschiedenis een compleet cardiovasculair consult aangeboden wordt onder supervisie van een obstetricus, een cardioloog en een vasculaire geneeskundige. Dit betekent dat de bevindingen uit dit proefschrift al op korte termijn consequenties hebben voor de dagelijkse klinische praktijk. 


\section{Nederlandstalige samenvatting}

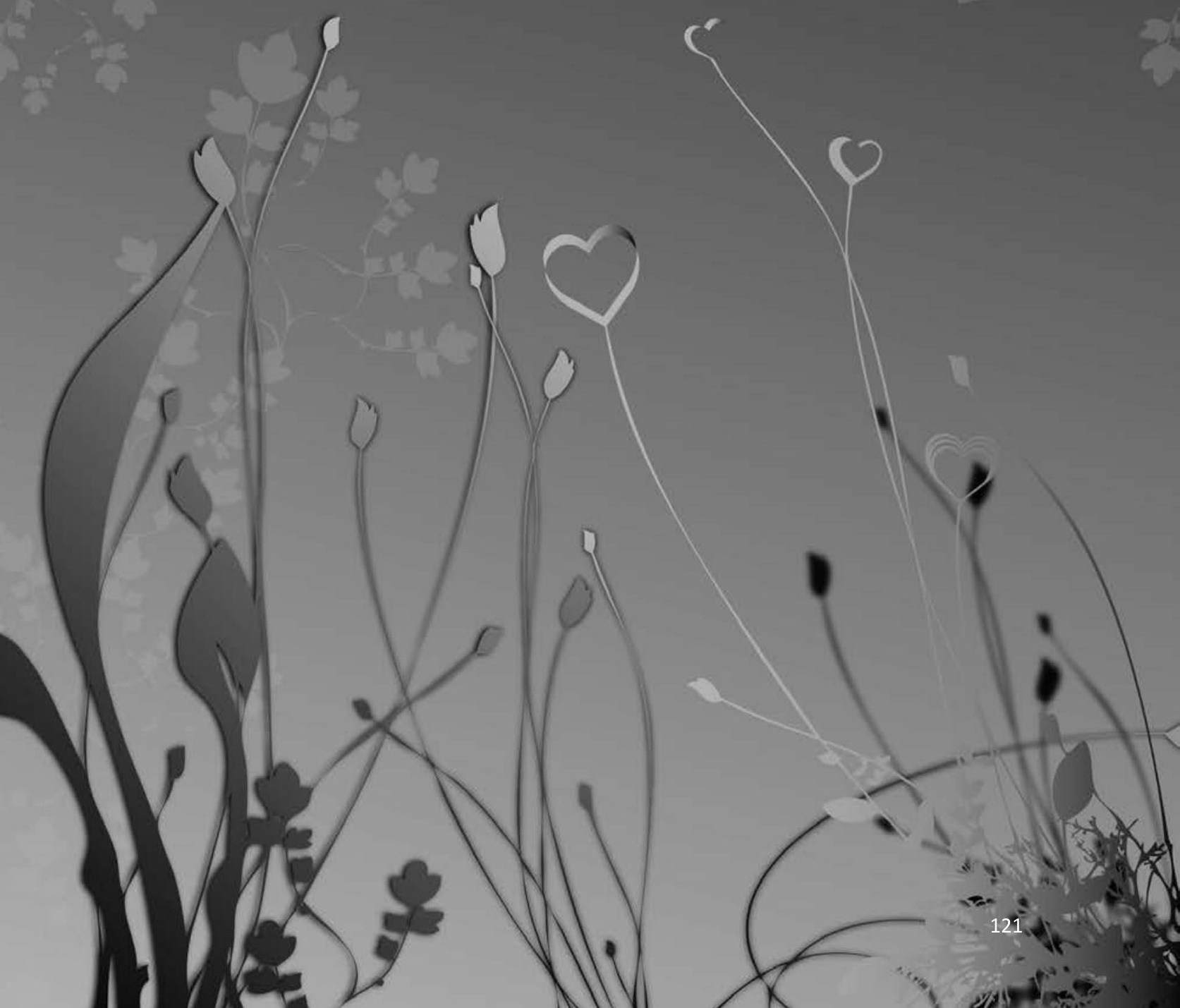




\section{Samenvatting}

Dit proefschrift beschrijft de studies betreffende de lange termijn gevolgen van preeclampsie (PE) op het hart en de cardiovasculaire gezondheid. Hoofdstuk 1 geeft een introductie over de achterliggende problemen die aan de vraagstellingen van dit proefschrift ten grondslag liggen. Zwangerschap wordt gezien als vrouw specifieke stresstest voor hart en vaten. Vaatproblemen tijdens de zwangerschap komen tot uiting door hoge bloeddrukproblemen zoals PE enerzijds en groeibeperking anderzijds en verraden een twee tot acht voudig verhoogd risico op hart en vaatziekten bij vrouwen. PE en hart- en vaatziekten hebben vaak gezamenlijke risicofactoren zoals obesitas, hypertensie en diabetes mellitus waardoor er vaak gedacht wordt dat er een overlap is in de pathogenese van beide ziektes. Het hart verandert tijdens zwangerschapsvergiftiging op een ongunstigere manier dan tijdens een probleemloze zwangerschap. Tijdens een normale zwangerschap wordt het hart groter en de wand dikker met daarbij een evenredige vergroting van de diameter van het linker ventrikel. Deze aanpassing wordt eccentrische remodelering genoemd. Tijdens PE wordt het hart groter en de wand dikker zonder een evenredige toename in de diameter van het linker ventrikel. Deze laatste aanpassing wordt concentrische remodelering genoemd. Dezelfde vorm van remodelering treedt ook op bij hartfalen. Dit proefschrift geeft antwoord op de vraag hoe na een doorgemaakte PE, het hart zich in een tweede zwangerschap aanpast, hoe het hart zich aanpast in de 14 jaar na PE en hoe deze aanpassingen gerelateerd zijn aan het ontwikkelen van hypertensie en hartfalen.

In het eerste deel van dit proefschrift wordt ingegaan op de aanpassing van het hart aan een tweede zwangerschap bij vrouwen met PE in de voorgeschiedenis en op de leeftijdsgebonden aanpassingen van het hart tot 14 jaar postpartum. Hoofdstuk 2 beschrijft de aanpassingen van het hart bij een groep vrouwen die een vroege PE hebben doorgemaakt in het verleden. Deze groep vrouwen kreeg op vier momenten metingen van het hart- en vaatstelsel. De eerste meting vond plaats voor de tweede zwangerschap. De tweede, derde en vierde meting vonden plaats in de eerste helft van de tweede zwangerschap. Van de deelnemende vrouwen is aan het einde van de zwangerschap de uitkomst van die zwangerschap nagevraagd en de groep werd verdeeld in een groep vrouwen die een tweede keer PE heeft ontwikkeld en een groep die de tweede keer een gezonde zwangerschap heeft doorgemaakt. In dit hoofdstuk wordt aangetoond dat de linker ventrikel massa van het hart bij vrouwen die een tweede PE ontwikkelen kleiner is dan bij vrouwen die de tweede keer een normale zwangerschap ontwikkelen, met daarbij een kleiner slagvolume en een hogere hartslag. Dit verschil bleef aanwezig tot een amenorroeduur van 20 weken. In Hoofdstuk 3 wordt bij een groep vrouwen die PE heeft gehad en bij een groep vrouwen zonder PE het hart onderzocht 1 jaar en 14 jaar postpartum. Hiermee werd de vraag onderzocht of de leeftijdsgeboden veranderingen van het hart anders en ongunstiger is bij vrouwen met PE in de voorgeschiedenis ten opzichte van vrouwen zonder PE in de voorgeschiedenis. Hiermee wordt aangetoond dat de leeftijdsgebonden aanpassing van het hart bij vrouwen met PE in de voorgeschiedenis in de daaropvolgende 14 jaar lijkt op die van vrouwen met enkel gezonde zwangerschappen in de voorgeschiedenis. In dit hoofdstuk hebben we geen 
onderscheid gemaakt in verschillende fenotypes na PE en konden dan ook niks zeggen over de aanpassingen van het hart bij de subgroep die hypertensie ontwikkelt.

In deel $\mathbf{2}$ van dit proefschrift wordt onderzocht in hoeverre bepaalde risicofactoren en cardiale waarden gerelateerd zijn aan hypertensie en hartfalen stadium-B. Hoofdstuk 4 beschrijft bij een groep vrouwen met $\mathrm{PE}$ in de voorgeschiedenis, welke risicofactoren en cardiale variabelen gerelateerd zijn aan het ontstaan van hypertensie in initieel normotensieve vrouwen. Het blijkt dat bij voormalig PE vrouwen, een vergrote linker ventrikel massa, een verhoogde diastole bloeddruk en de aanwezigheid van prehypertensie, alle drie sterk gerelateerd zijn aan het ontwikkelen van hypertensie in de daaropvolgende 12 jaar.

Hoofdstuk 5 toont de resultaten van een longitudinale pilotstudie waarbij vrouwen met PE in de voorgeschiedenis cardiovasculaire metingen hebben ondergaan 1 jaar postpartum en 14 jaar postpartum. Bij 14 jaar postpartum werd de onderverdeling gemaakt in een groep vrouwen die hypertensie heeft ontwikkeld en een groep vrouwen die geen hypertensie heeft ontwikkeld. In dit hoofdstuk wordt aangetoond dat de groep met hypertensie vaker, al 1 jaar postpartum een mild verhoogde bloeddruk heeft ten opzichte van de groep vrouwen die 14 jaar later geen hypertensie heeft ontwikkeld. Daarnaast heeft de groep met hypertensie 14 jaar later een grotere ratio tussen wanddikte en diameter van het linker ventrikel en een slechtere ontspanfunctie van het hart.

Hoofdstuk 6 toont aan dat in het eerste decennium na een zwangerschapsvergiftiging bij 1 op de 4 vrouwen een asymptomatische vorm van hartfalen (hartfalen stadium-B) voorkomt. Hartfalen is de belangrijkste veroorzaker van sterfte bij vrouwen. HF stadium-B is een voorloper van de klinische vorm van hartfalen. De overgang van subklinisch naar klinisch hartfalen gaat gepaard met een vijfvoudige stijging in gerelateerde sterfte. Daarnaast zijn een mild verhoogde bloeddruk, hogere BMI en een lager HDL geassocieerd me deze aandoening.

In Hoofdstuk 7 worden de bevindingen van dit proefschrift bediscussieerd. De studies in dit proefschrift tonen een hoge prevalentie aan van prehypertensie, aanhoudend vergrote linker ventrikel massa en bepaalde componenten van het metabool syndroom na preeclampsie. In dit proefschrift tonen wij aan dat deze risicofactoren geassocieerd zijn met chronische hypertensie en hartfalen stadium-B later in het leven. Het doormaken van preeclampsie zou de clinicus moeten attenderen op het aanpassen van de zorg en om cardiovasculair risicomanagement aan te bieden met minstens regelmatige bloeddruk controles om secundaire preventie te kunnen toepassen.

Tot slot wordt in Hoofdstuk 8 ingegaan op de valorisatiemogelijkheden van de onderzoeksresultaten, de gebruikte cohorten en de ideeën die ontstaan zijn tijdens de studieperiode. Pre-eclampsie (PE) kan gezien worden als een vrouwspecifiek risico indicator die op jonge leeftijd de mogelijkheid biedt om vrouwen met een verhoogd risico op HVZ te identificeren en op een multidisciplinaire poli cardiovasculaire management aan te bieden. Omdat deze groep vrouwen een hoge prevalentie subklinisch hartfalen heeft, biedt deze groep vrouwen mogelijkheid om onderzoek te verrichten naar veelbelovende biomarkers die in een vroeg stadium vrouwen met een verhoogd risico op hart- en Vaatziekte kunnen opsporen. 



\section{Dankwoord}

Dit proefschrift vat in een aantal hoofdstukken het onderzoek samen waar ik de afgelopen jaren aan heb gewerkt. Het weerspiegelt voor mij niet alleen mijn wetenschappelijke ontwikkelingen van de afgelopen jaren, maar ook enkele persoonlijke ontwikkelingen waarbinnen het nodige is gebeurd. De veranderingen in de afgelopen jaren traden in een razend tempo op en deze doorliep ik samen met al mijn dierbaren in verschillende versnellingen en met afwisselend hoogte- en dieptepunten. Mijn heugelijk wachten werd de afgelopen jaren met enkele teleurstellingen beantwoord. Vreugde en verdriet werden dagelijks al tossend ingezet. Wat zou de dag meebrengen, welke wind zou er gaan waaien...? Deze afwisseling was zo frequent dat het bijna een monotoon karakter kreeg. Het was een lange 4 jaar durende herfst. Zwaar, wispelturig, nat....maar lang, vrij lang. En dat heb ik allemaal volgehouden, bedenk ik me nu, om dit proefschrift af te ronden.

Deze stormachtige herfst had ik niet aangekund zonder een aantal geweldige mensen om mij heen. 'In zware tijden leer je je vrienden echt kennen' was meer dan ooit op mij van toepassing, maar dan zonder de negatieve bijklank. Ik heb de schoonheid van onvoorwaardelijke liefde en vriendschap dankzij jullie mogen ervaren. En dat is een zegen die voor mij opweegt tegen alle tegenslagen van de afgelopen jaren.

Allereerst draag ik deze thesis op aan ons engeltje onderwiens vleugels ik bescherming heb gevonden tegen alle onverwachte ontwikkelingen. Rachelle, jij bent de eerste kaars die in ons gezin aangestoken werd. Helaas is deze kaars snel gaan doven, maar de warmte die ik heb gekend door jou in mijn armen te sluiten, is de warmte die me nog steeds warm houdt. Ik mis je, engeltje.

\section{Lieve Mazen,}

Een vrolijk lachebekje vanaf de allereerste dag. Dankzij jou heb ik leren relativeren. Aan het einde van de dag geven jouw opgewektheid, vreugde en liefde mij weer energie. Ik geniet van je koppigheid en eigenwijsheid. Twee mannen in huis die aan een paar uur slaap per nacht voldoende hebben, dat belooft nog iets de komende jaren. Papa en ik zijn bijzonder trots op je en we beloven je alle liefde van de wereld.

\section{To Karim, my one and only, Habibi}

The past years where not always as easy as we wished for. Ten years where filled with the best time of our life, followed by the worst time. A lot of things happened that were out of our hands. We passed it and turned the bad into good. We grew stronger and our bond grew stronger. You are able to motivate me and support me during difficult, times. Karim, as a manager by nature you knew how to manage our home and our (rare) time. But please remember honey, for domestic affairs, I'm still in charge! 


\section{Lieve Mama en Papa}

Jullie zijn de drijfveer achter mijn besluit om geneeskunde te gaan studeren. Het doel was gynaecoloog te worden. Dat was jullie wens en daarmee mijn wens al die tijd geweest. De onvoorziene omstandigheden makten dat dit geen wens meer was. De passie is overgeslagen naar de cardiologie en als het aan mij ligt, vrouwspecifieke cardiologie.

De diversiteit in jullie interesses, met name politiek, literatuur en cultuur, werken aanstekelijk en inspireren mij. Papa, jij hebt overgebracht dat "the sky should be the limit and nothing less". Jij was de wijsheid, beheersing en kracht die achter elke stap school.

Mama, je liefde en zorg hebben me warm gehouden de afgelopen jaren. Jouw liefde voor kennis was voor mij aanstekelijk en drijfveer geweest om dit promotieonderzoek vol te houden. Bedankt voor de steun, zorg en liefde die jullie aan Mazen geven. Met deze steun was het een stuk gemakkelijker om door te zetten.

\section{Prof. Spaanderman, beste Marc,}

Een jaar na het starten van mijn promotietraject kwam jij in beeld in Maastricht en na enkele maanden gingen we nauwer samenwerken. De uren aan telefoongesprekken met jou koester ik. Wat hebben wij toch uren gebeld en wat heb ik veel geleerd van onze gesprekken. Jij bezit het zeldzame vermogen om een ander toch de andere kant van de medaille te laten zien. Soms duurde het wel even, maar in veel gevallen lukte het je ook bij mij (ja zelfs bij mij). In veel zijn wij elkaars tegenpolen. Als mijn gemoederen hoog oplopen, breng jij mij tot bedaren. Als ik ongeduldig wordt doordat ik het niet snel genoeg vind gaan, zet jij me weer met beide voeten op de grond. Als leermeester in de wetenschap heb je me alle ruimte gegund om mijn eigen denkwijze vorm te geven. Bedankt voor de inspiratie en voor je vriendschap. Opdat we nog vele uren kunnen bellen en vele jaren kunnen samenwerken.

\section{Dr. Peeters, beste Louis,}

Ik leerde je kennen als facultaire begeleider voor mijn keuzecoschap in Dubai, waarna ik je heb benaderd voor een WESP stage. Ik wilde zo snel mogelijk de kliniek in, maar jij hebt het me afgeraden en me overtuigd me eerst te gaan richten op promoveren. De argumenten waren het bekende mes en vork verhaal en het kookboekjesgeneeskunde verhaal. Ook zal er nog genoeg tijd zijn voor het sein en de witte jas dus waarom de haast? Je had me overtuigd. Maar er was een probleem: geen onderwerp en geen geld. Al snel kwamen de oplossingen. We kregen een onderwerp en we kregen geld, met daarbij een aantal andere problemen. Al die jaren heb je het voor me opgenomen als een vader voor zijn dochter. Ik kon achter jou schouders blijven staan en daar ben ik heel dankbaar voor. Bedankt voor je vertrouwen en dat ik op je kon bouwen. De prijs voor beste mentor die je gekregen hebt op de SGI in Miami heb je dubbel en dwars verdiend. Oprecht kan ik zeggen dat er zonder jou geen start was gekomen aan dit promotietraject maar ook geen einde. 


\section{Beste leden van de Leescomissie}

Beste Prof. dr. P.W. de Leeuw, Prof. dr. H.P. Brunner-La Rocca, Dr. S.V. Koenen, Prof. dr. A. H. E. M. Maas, Prof. dr. J.G. Nijhuis. Bedankt voor het inzetten van jullie aller expertise in het beoordelen van mijn proefschrift. Ik kijk er nu al naar uit om mijn proefschrift te verdedigen met $u$ als opponent.

\section{Lieve Sousou, Moedi en Mahdi, en Ahmad natuurlijk}

Ons gebrabbel in half Nederlands/ half Arabisch. Veel van onze gesprekken en zinnen snappen wij alleen. "2aymta jeye naar mij toe?" "Bas trouh 3al supermarkt, vergeet almelk, het brood, en ba2dounes niet." "Khebes khesnijden; als hij en zij jij niet blijf pleister; Coach/kwatch."

Wat hebben wij toch vaak de slappe lach. Sous, ik kan er niks aan doen dat mijn Engelse accent mooier klinkt dan dat van jou. Maar een jaartje in de States zal je accent zeker goed doen, vermoed ik. Ik zal je wel gaan missen. Qatar leek nog dichtbij, maar de VS is toch wat verder vliegen. Gerust ben ik erom dat je daar samen "wiz" Ahmad bent. Ahmad, I'm confident that you will fulfill your professional dreams in the USA. I admire your intelligence and ambition. I'm sorry for all the snowplowing that Karim made you do and for sending you in the highest trees in the garden. You know we love you as a brother!

Moedi, ik kan er niets aan doen dat ik knapper ben dan jij. Ik weet dat je er veel moeite mee hebt, maar goed, je kunt niet alles mee hebben in het leven. Ik ben trots op je nerdy prestaties in je studie en ik weet zeker dat je een uitstekende dokter zult worden.

En Mahdi, lief klein allerlangste broertje. Ik breng je graag naar voetbal, en karate, en school (als je fiets weer eens gestolen of kapot is, of om welk ander reden dan ook op dat moment niet beschikbaar is). Maar als ik in de toekomst voor een kruispunt sta, en ik vraag je of ik links of rechts af moet, geef dan alsjeblieft antwoord en zeg niet "raad maar". Ondanks dat jij mij soms dol maakt, ben ik toch ontzettend dol op je. En nee, jij en Moedi zijn niet zo geboren. Ow ja....wanneer gaan jullie eindelijk eens je eigen boterhammen smeren?!

\section{Dear tante Majida, Amer, Mazen, Sibilla, Jaayar, Hadi and Daniella,}

You became my second family through Karim. Although each of us lives in a different country, it feels that we are all close to each other. I am sorry that Amo could not be amongst us on special days like this, but his spirit will always be around. Amer, I hereby admit that your food is delicious. Even without a cooking machine (I suppose). And Sibilla, I admire your commitment as a doctor, you are a big example for me. Mazen, I found in you an elder brother who makes the family feel safe. Jaayar, it's always fun with you around, I will never forget our trip to Italy and how much we laughed. We are waiting for your next visit in July to spend time with Hadi, Daniella and Mazen jr. in the Efteling. 


\section{Lieve Anila, Aisha, Sabah en Naoual,}

Mijn studiemaatjes, vrienden en praktisch familie. Vanaf het eerste jaar geneeskunde samen en nog steeds samen en inchallah altijd samen. Bij de geboorte en overlijden van Rachelle hebben jullie me geen moment alleen gelaten. Het leek wel of jullie een dienstenrooster hadden gemaakt om ervoor te zorgen dat er altijd wel iemand aanwezig was of aan de telefoon hing om me af te leiden. Door drukte hebben we elkaar de laatste tijd minder vaak gezien, maar daar gaan we weer verandering in brengen. Laten we hier en nu een datum prikken om de stad weer onveilig te maken. Aisha, we laten de kinderen wel bij de mannen en gaan een dagje on our own. Anila, binnenkort kom ik weer logeren, maar nu eens een keer niet om voor de stationtoets te leren. En Sabah, jij rijdt iedere dag langs, ik ga niet eens moeite doen om een datum te prikken. Raak niet te geïntegreerd en kom gewoon onaangekondigd binnenvallen. En Naoual, het wordt tijd dat je nu van dat roze wolkje af komt. Er is nog een leven hier down on earth!

\section{Mijn paranimfen Ellen en Sander,}

Dat was wel even nadenken. Zou ik Ellen en Siamack, en Sander en Leandra samen noemen? Of toch Ellen en Sander als mijn paranimfen? Ik heb voor het laatste gekozen. Van alle collega's zijn jullie mijn echte onderzoeksmaatjes en vrienden geworden. Wij drieën hebben gemeen dat we zo van het onderzoek en elkaars gezelschap en gesprekken kunnen genieten, ondanks dat we allen op een ander onderwerp zaten.

Sander, bij onze eerste MANOVA (of was het toch ANCOVA?), klikte het al. Jij gaat net zo charmant en makkelijk om met statistiek als met mensen. Dat is een waardevolle combinatie die niet velen je kunnen evenaren. Ik heb veel van je geleerd over statistiek en methodologie. Ik hoop dat wij nog aan vele mooie projecten mogen samenwerken. Dankzij jou heb ik Leandra leren kennen en ook met jou klikte het snel. Je creativiteit en energie werken erg aanstekelijk en zijn enorm inspirerend. Je bent een kei in wat je doet, powervrouw!

Ellen, als ik probeer terug te denken aan hoe wij vrienden zijn geworden, kan ik me geen specifiek moment voor de geest halen. Het is zo gegaan alsof het de meest natuurlijke zaak van de wereld is. Je bent een grote steun voor me geweest en hebt me vaak een luisterend oor aangeboden. Al snel hebben jij, Anila, Eveline en ik de boekenclub opgericht. Of zullen we zeggen een bijklets, bij-eet en -drink club? Ook jij, Siamack, stond altijd voor me klaar. Ik hoefde maar te 'appen' en je was me al voor op de $4^{\text {de }}$ van het AZM. Als Kootstra- en Mozaiekmaatje delen wij de passie voor onderzoek en de ambitie voor verder. Ik ben ervan overtuigd dat je het nog ver gaat schoppen.

\section{Het voormalige PERIM team,}

Inez, met jou heb ik het meest mogen samenwerken. Als een PERIM-moeder heb je voor de hele groep en elke onderzoeker in de groep de afgelopen jaren gezorgd. We missen je nu en missen je warme karakter. 
Timo en Hennie, ik heb maar even met jullie kunnen samenwerken, maar jullie bijdragen aan de data collectie is ongetwijfeld essentieel geweest voor dit en vele proefschriften die uit PERIM voortvloeien.

Julia, Simone en Carmen, jullie zijn mijn directe voorgangers geweest als promovendi bij PERIM. Met elk van jullie heb ik kort en op een ander moment samengewerkt. Het waren geen lange periodes, maar jullie hebben zeker je nadruk achtergelaten.

\section{Het huidige PERIM team,}

Als ik vorig jaar dit dankwoord had geschreven, had ik hier niet veel namen hoeven noemen. Maar nu: Eva, Veronica, Nicki, Stéphanie, Ghislaine, Jolien, Mieke, Jonas. Wow, wat zijn we gegroeid met zoveel geweldige onderzoekers en wat zijn jullie in de afgelopen periode gegroeid. Allen met jullie eigen talenten en onderzoeksvragen. Een groep waar je op kunt bouwen. We mogen trots op onszelf zijn. Bedankt voor jullie geduld, steun en begrip bij drukte. Succes met jullie eigen studies en promotietrajecten!

\section{Mijn collega's van de afdeling BMT}

Beste Arnold, Theo, Koen, Joost, Bart, Marieke, Yvette, Jeroen, Wouter, Jeire en Alex. De innerverende periode die ik heb gehad op de afdeling BMT doet niets af aan wat ik van jullie heb geleerd en de gezellige momenten die we hebben gehad. Ik zal nooit vergeten hoe tijdens de pauzes, gewoon ter ontspanning, fysische vraagstukken worden opgelost. En de weg kwijt raken in Brussel is nooit eerder zo leuk geweest als met ons groepsuitje. Succes met al jullie projecten.

\section{Beste Jolijn,}

Samen hebben wij de HaVaNa studie opgezet en dat is voor mij het voorbeeld geweest van hoe leuk onderzoek kan zijn. Het was veel werk en het was druk, maar we hebben het met veel lol gedaan. Ik vind het jammer dat wij niet meer samenwerken, maar Sushi eten zullen we nog lang samen blijven doen onder het genot van een portie gediss!

\section{Beste dr. Scheepers, beste Liesbeth,}

Tijdens mijn zwangerschap heb je niet alleen mijn controles gedaan, maar ben je ook een enorme steun geweest voor alles wat ik doormaakte. Een geweldig betrokken en empathische arts, eentje waar ik graag voorbeeld aan wil nemen. Bedankt voor alle steun en voor het luisterend oor!

\section{TVDC team,}

Het hele team van het TVDC, Alfred, Caroline, Stella, Gonny, Betul, Marie-Jeanne, Nathalie, Eri, Yvonne. Een geweldig team dat zorg en wetenschap waarbinnen de vrouw centraal staat integreert op het transmuraal vrouwen dagcentrum. Veel van de data voor onze studies worden door jullie mogelijk gemaakt. Ook is de begeleiding die 
door jullie geboden wordt aan studenten iets waar we trots op zijn. Bedankt voor jullie betrokkenheid!

\section{Beste Salwan en Narjes}

Collega's, buren en vrienden. Salwan, het is leuk om te zien hoe je bruist van de ideeën. Het kan voor jou niet snel genoeg gaan en je slaat graag spijkers met koppen. Je bent volop je eigen onderzoekslijn aan het opzetten en het gaat prima. Ben blij dat ik daar onderdeel van uitmaak.

Narjes, jouw liefde voor verrassingen, feestjes en heerlijk eten is zo bijzonder. Ik bewonder enorm hoe je het moederschap van 3 kinderen combineert met je drukke promotietraject. Dat is niet makkelijk maar beide doe je voortreffelijk. Komt de ooievaar deze zomer ook langs om aardbeien te brengen? ;)

\section{Beste Randa en Raed,}

Jullie zijn een aanvulling voor de familie. Jullie zachtheid en betrokkenheid zijn voor ons onmisbaar geworden. Randa, ik ben blij dat ik van jou heb geleerd hoe ik een lamsbout heerlijk kan klaarmaken. En Raed, ik hoop dat wij in de toekomst nog veel gaan samenwerken!

\section{Lieve Zlatan en Eveline}

Zlatan, vanuit de studie zijn we binnen no time vrienden geworden. Ook gingen we als studenten op de huisartsenpost werken samen met Eveline, Anila, en Rik. Al snel begonnen we allebei aan een huisje, boompje, beestje. En de vriendschap sloeg ook over naar Eveline en Karim. We maakten van onze roulerende dinerpartijtjes traditie. Heerlijk afwisselde Bosnische, Libanese, Italiaanse en Portugese gerechten. Karim en jij mogen altijd meehelpen met afruimen, maar laat het koken gewoon aan Eveline en mij over.

\section{Skillslab Collega's}

Naast mijn promotietraject heb ik onderwijs via het skillslab mogen geven. De meesten van jullie kende ik als studente al. De omslag van een student-docent naar een collegacollega relatie was moeilijk voor mij. Vele van jullie heb ik als voorbeeld genomen voor hoe ik als docent wilde zijn. De mogelijkheden voor ontplooiing op het skillslab heb ik enorm gewaardeerd. Ook jullie betrokkenheid bij eenieders persoonlijke omstandigheden is iets om te koesteren. Het is geen vanzelfsprekendheid weet ik inmiddels. Dank voor alle fijne, warme en leerzame momenten.

\section{Stefan}

Al vanaf de basisschool was jouw tekentalent opvallend. Een kunstenaar bij geboorte. Ik had er nooit bij stilgestaan dat jij met dat talent het geboortekaartje van Rachelle zou ontwerpen. Dat we die niet zouden hoeven afdrukken maar dat we het na kleine aanpassingen zouden gebruiken voor het kaft van dit proefschrift. Maar toch is het zo 
gegaan. En ik ben blij dat jij het voor mij hebt gedaan. Ik vind het niet alleen prachtige kunst, het betekent ook veel voor me. 

Chahinda Ghossein is geboren op 23 december 1985 in Zuid Libanon. In maart 1990 emigreerde zij op vierjarige leeftijd met haar ouders en zusje als politieke vluchtelingen naar Nederland. Zij groeide op in Roermond waar ze het VWO voltooide op het Bisschoppelijk College Schöndeln. In 2004 begon ze aan de studie geneeskunde aan de Universiteit van Maastricht. Aansluitend aan het behalen van het artsenexamen in augustus 2010 werd haar de Kootstra Talent Fellowship toegekend waardoor ze het voortraject van haar promotieonderzoek bij de vakgroep Gynaecologie en Obstetrie kon initiëren, onder begeleiding van dr. Louis Peeters. In hetzelfde jaar won zij op het SGI congres de Pfizers President Presenter Award.

Haar passie voor onderwijs maakte dat ze naast haar promotietraject via het skillslab een combinatieaanstelling kreeg waardoor ze diverse onderwijsrollen heeft kunnen verzorgen aan de FHML-geneeskunde opleiding. Hiervoor ontving zij in 2011 de Onderwijsprijs. Vervolgens werd haar in 2012 de Mozaïek beurs van de NWO toegekend waardoor ze onder begeleiding van dr. Louis Peeters en prof. Marc Spaanderman, haar onderzoek in de daaropvolgende 4 jaar kon voortzetten. Haar loopbaan werd enkele malen door professionele en persoonlijke struikelblokken onderbroken maar haar doorzettingsvermogen en liefde voor onderzoek en de steun van de mensen om haar heen zorgde dat ze met meer kracht en motivatie zich steeds herpakte.

Aansluitend aan haar promotieonderzoek is ze tot op heden werkzaam als onderzoekster bij de Queen of Hearts studie. Dit zal ze het komend jaar voortzetten, om vervolgens een klinische carrière bij de Cardiologie te starten. Voor sommigen een carrièreswitch, voor anderen een logische voortzetting van de expertise die ze tijdens dit promotieonderzoek heeft opgedaan. Chahinda is getrouwd met Karim Doha, en is moeder van twee kinderen: dochter Rachelle die ze altijd in haar hart meedraagt en uit wiens gedachte ze kracht put, en zoontje Mazen die haar leven vult met vreugde en liefde. 
Chahinda Ghossein was born on December 23rd 1985 in South-Lebanon. On March 1990, at the age of 4 years, she immigrated with her parents and sister to the Netherlands, as political refugees. She grew up in Roermond and completed high school at the Bisschoppelijk College Schöndeln. In 2004 she started medical school at the University of Maastricht. Shortly after graduating, she was granted the Kootstra Talent Fellowship and she started her research at the department of Obstetrics and Gynaecology, under supervision of dr. Louis Peeters. In the same year she also won the Pfizers President Presenter Award at the SGI conference.

As a teacher in the Skillslab, she combined her scientific work with her passion for teaching and education. She fulfilled several educational roles at the faculty of Health Medicine and Life Sciences of the MUMC+ and in 2011 she received the education price. Next, she received in 2012 the Mosaic Fellowship from the NWO to continue her research in the subsequent 4 years under supervision of $\mathrm{dr}$. Louis Peeters and prof. Marc Spaanderman. Her career was discontinued a few times due to personal and professional reasons but her persistency and love for her research and the support of the people around her made her continue to achieve her goal.

She is currently working as a researcher at the Queen of Hearts research which she will continue the coming year after which she will start her clinical career at the department of cardiology. Some may think it as a career switch some see it as a logic continuum of her research. Chahinda is married to Karim Doha, and is mother of two children: a daughter Rachelle who she always carries with her in her hart and thoughts and who gives her strength, and a son Mazen who fulfils her life with happiness and pride. 


\section{Publications}

Age related alterations in cardiac geometry in formerly preeclamptic women and healthy parous controls: an explorative study; Ghossein-Doha C; van Kuijk S; Spaanderman ME; Delhaas T; Peeters LL; reprod Sci.2013 Jan;20(1):39-44

- $\quad$ Early pregnancy circulatory adaptation and recurrent hypertensive disease; Lopez van Balen; Spaan J; Ghossein C; van Kuijk S; Spaanderman ME; Peeters LL; reprod Sci 2013 Aug.

- $\quad$ Hypertension after preeclampsia is preceded by changes in cardiac structure and function; Ghossein-Doha C; Peeters LLH, van Heijster S; van Kuijk; Spaan J; Delhaas T; Spaanderman ME; Hypertension 2013 Aug;62(2):382-90

- Long-term risk to develop hypertension in formerly preeclamptic women: A longitudinal pilot study; Chahinda Ghossein-Doha, Marc Spaanderman,, Sander MJ van Kuijk, A.A. Kroon, Tammo Delhaas,, and Louis Peeters; Reproductive Sciences January 18, 2014

- Metabolic syndrome after pregnancy complicated by preeclampsia or small for gestational age infancy; Salwan Al-Nasiry, Chahinda Ghossein-Doha, Sharona EJ Polman, Stephanie Lemmens, Ralph R Scholten, Wieteke M Heidema, , Julia J Spaan, Marc EA Spaanderman; BJOG october 16,2014 DOI: 10.1111/14710528.13117

- $\quad$ Cardiovascular disease risk is only elevated in hypertensive formerly preeclamptic women; NM Breetveld, C Ghossein-Doha, SMJ van Kuijk, AP van Dijk, MJ van der Vlugt, RR Scholten, WM Heidema, MEA Spaanderman; BJOG augustus 20, 2014; doi: 10.1111/1471-0528.13057

- Cardiac adaptation to the next pregnancy in formerly preeclamptic women bound to develop recurrent preeclampsia; Chahinda Ghossein-Doha,, Marc M.E. Spaanderman, Rahmah Al Doulah, Sander M.J. van Kuijk, Tammo Delhaas, Louis Peeters (Submitted UOG)

- Preeclampsia, an independent risk factor for asymptomatic Heart Failure; C Ghossein-Doha, J van Neer, B Wissink, LJ de Windt, APJ van Dijk, MJ van der Vlugt, MD, PhD, MCH Janssen; MD PhD; WM Heidema; MD, RR Scholten, MD, MEA Spaanderman; (submitted to journal of Obstetrics and Gynecology)

- Maternal metabolic syndrome, preeclampsia and small for gestational age infancy; M. Hooijschuur; C. Ghossein-Doha; S. Al Nasiry; M. Spaanderman (accepted BJOG)

- $\quad$ Heart failure in former preeclamptic women at one and four years postpartum; Nicolette M Breetveld, Chahinda Ghossein-Doha, Sander MJ van Kuijk, Arie P van Dijk, Maureen J van der Vlugt, Wieteke M Heidema, Jolijn van Neer, Vanessa van Empel, MD, Hans-Peter Brunner-La Rocca, MD, PhD, Ralph R Scholten, Marc EA Spaanderman, (Submitted to JACC) 


\section{Manuscripts in preparation}

- The significance of placental hypoechoic lesions in normal and high-risk pregnancies: a meta-analysis. G.H.M.W. Haarsma-Gruiskens, Andrew Moufarey, C. Ghossein-Doha, A. Coumans, S. Van Teeffelen, S. van Kuijk, M. Spaanderman, S. Al-Nasiry

- $\quad$ Decreased PV and increased peripheral vascular resistance soon after PE relate to the development of remote asymptomatic HF; Chahinda Ghossein-Doha, Nicolette M Breetveld, Sander MJ van Kuijk, Arie P van Dijk, MD, Maureen J van der Vlugt, MD, Wieteke M Heidema, Jolijn van Neer, Vanessa van Empel, HansPeter Brunner-La Rocca, MD, Ralph R Scholten, Marc EA Spaanderman, MD

- $\quad$ Cerebroplacental ratio in pregnancies complicated by placental syndrome; $M$. Nijhuis; G.H.M.W. Haarsma-Gruiskens, C. Ghossein-Doha, M. Spaanderman, S. Al-Nasiry

Identifying women with metabolic syndrome after preeclamptic pregnancies; Maternal metabolic syndrome after preeclamptic pregnancies; the influence of onset disease and small for gestational age infancy; M. Hooijschuur; C. GhosseinDoha; S. Al Nasiry; M. Spaanderman 


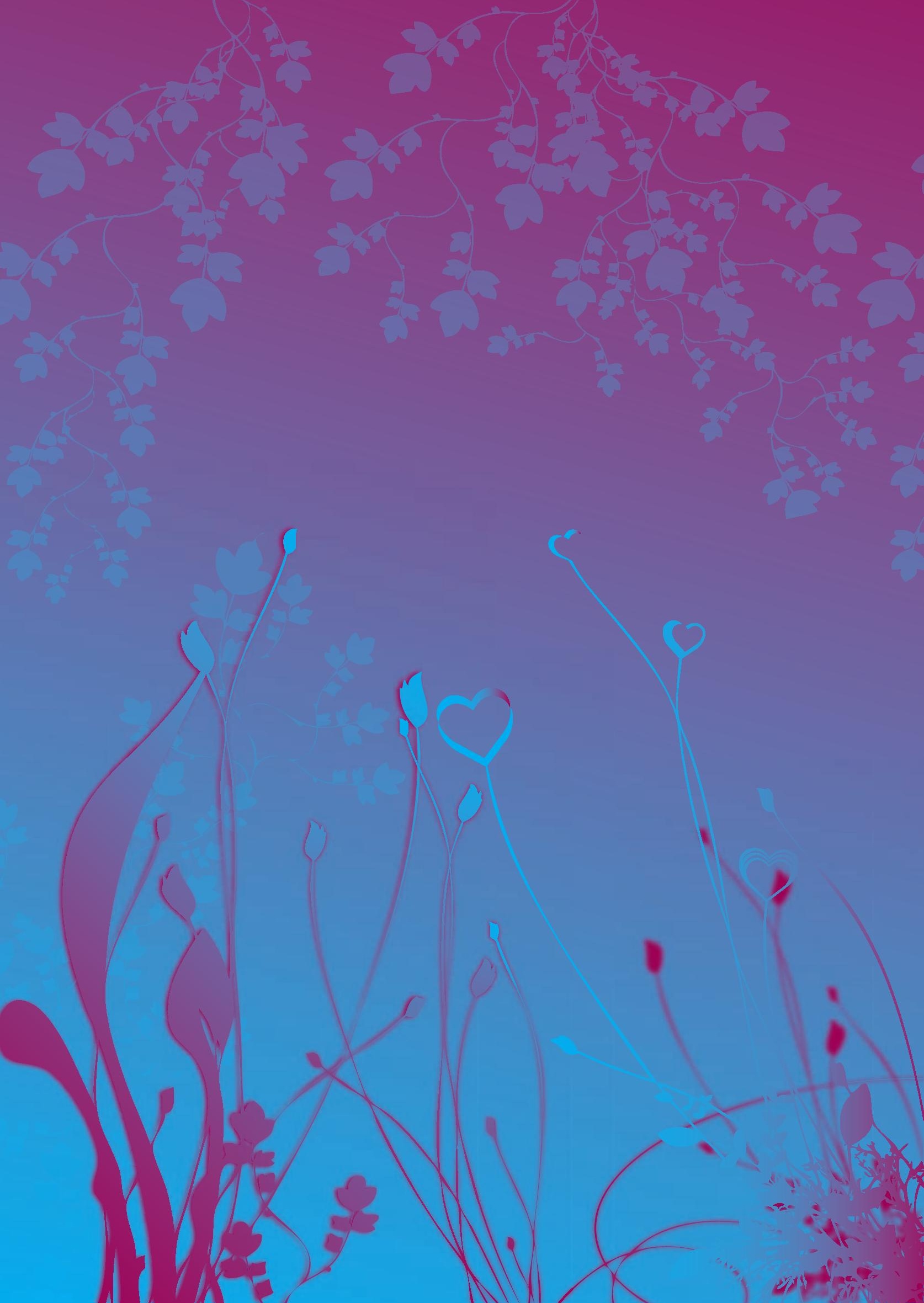

